\title{
Conformal transformations of the pseudo-Riemannian metric of a homogeneous pair
}

\author{
Kotaro Kawai *
}

\begin{abstract}
We introduce a new notion of a homogeneous pair for a pseudo-Riemannian metric $g$ and a positive function $f$ on a manifold $M$ admitting a free $\mathbb{R}_{>0^{-}}$ action. There are many examples admitting this structure. For example, (a) a class of pseudo-Hessian manifolds admitting a free $\mathbb{R}_{>0}$-action and a homogeneous potential function such as the moduli space of torsionfree $G_{2}$-structures, (b) the space of Riemannian metrics on a compact manifold, and (c) many moduli spaces of geometric structures such as torsion-free Spin(7)-structures admit this structure. Hence we provide the unified method for the study of these geometric structures.

We consider conformal transformations of the pseudo-Riemannian metric $g$ of a homogeneous pair $(g, f)$. Showing that the pseudo-Riemannian manifold $(M,(v \circ f) g)$, where $v: \mathbb{R}_{>0} \rightarrow \mathbb{R}_{>0}$ is a smooth function, has the structure of a warped product, we study the geometric structures such as the sectional curvature, geodesics and the metric completion (if $g$ is positive definite) w.r.t. $(v \circ f) g$ in terms of those on the level set of $f$. In particular, (1) we can generalize the result of Clarke and Rubinstein (CR2 ) about the metric completion of the space of Riemannian metrics w.r.t. the conformal transformations of the Ebin metric, and (2) two canonical Riemannian metrics on the $G_{2}$ moduli space have different metric completions.
\end{abstract}

\section{Introduction}

In this paper, we introduce a new notion of a homogeneous pair for a pseudoRiemannian metric $g$ and a positive function $f$ on a manifold $M$, possibly infinite dimensional, admitting a free $\mathbb{R}_{>0}$-action as follows.

Definition 1.1. Let $(M, g)$ be a pseudo-Riemannian manifold which admits a free $\mathbb{R}_{>0}$-action. Let $P \in \mathfrak{X}(M)$ be a vector field generated by the $\mathbb{R}_{>0}$-action. Suppose that $f: M \rightarrow \mathbb{R}_{>0}$ is a smooth function and $\alpha \in \mathbb{R}-\{0\}$.

The pair $(g, f)$ is called a homogeneous pair of degree $\alpha$ if

$$
m_{\lambda}^{*} g=\lambda^{\alpha} g, \quad m_{\lambda}^{*} f=\lambda^{\alpha} f, \quad g(P, \cdot)=d f,
$$

for any $\lambda>0$, where we denote by $m_{\lambda}$ the action of $\lambda>0$.

There are many examples admitting this structure. For example, a class of pseudo-Hessian manifolds admitting a free $\mathbb{R}_{>0}$-action such as the moduli

*2010 Mathematics Subject Classification. 58B20, 53C29, 58D17, 51F99. 
space of torsion-free $G_{2}$-structures. Hessian manifolds appear in many fields of mathematics such as information geometry ([AN, AJLS]) and the moduli spaces of geometric structures ([Hitchin1, Hitchin2]). The space of Riemannian metrics on a compact manifold, the moduli space of torsion-free Spin(7)-structures and many other moduli spaces of geometric structures also admit this structure. For more details, see Section 5 . Hence we provide the unified method for the study of these geometric structures.

Given a homogeneous pair $(g, f)$, we consider the conformal transformations of $g$ of the form $(v \circ f) g$, where $v: \mathbb{R}_{>0} \rightarrow \mathbb{R}_{>0}$ is a smooth function. There are two reasons to consider this.

(a) The conformal transformations of $g$ is considered in many examples such as the $G_{2}$ moduli space and the space of Riemannian metrics. See Sections 5.2 .3 and 5.3 .2

(b) When $g$ is positive definite and the pseudometric $d_{g}$ induced from $g$ is a metric (This is always true when $M$ is finite dimensional. In the infinite dimensional case, there are examples of a Riemannian metric whose induced pseudometric is identically zero $([\mathrm{MM}])$.), the conformal transformation is the simplest way to produce the different metric completion w.r.t. the induced metric.

Clarke and Rubinstein ([CR2] showed that there is an explicit weak Riemannian metric $\tilde{g}_{E}$ in the conformal class of the Ebin metric $g_{E}$ on the space of Riemannian metrics $\mathcal{M}$ such that the metric completion induced from $\tilde{g}_{E}$ is strictly smaller than that from $g_{E}$. They considered this as a first step to remove certain types of degenerations so that the canonical functionals such as the curvature, diameter, or injectivity radius are controlled by the metric geometry on $\mathcal{M}$, which is not true for the metric induced from $g_{E}$ (Clarke2 $)$.

We hope that generalizing this by using a homogeneous pair, which is done in Theorem 3.23, would be useful to remove certain types of degenerations for other geometric problems.

For the pseudo-Riemannian manifold $(M,(v \circ f) g)$, we first show that the following splitting theorem holds as in [Loftin, Theorem 1] and Totaro, Lemmas 2.1 and 2.4].

Theorem 1.2. Let $(M, g)$ be a pseudo-Riemannian manifold which admits a free $\mathbb{R}_{>0}$-action and let $f: M \rightarrow \mathbb{R}_{>0}$ be a smooth function. Suppose that $(g, f)$ is a homogeneous pair of degree $\alpha$. Then

- for any $l>0, M_{l}=\{x \in M \mid f(x)=l\}$ is a submanifold of $M$ and the pullback $g_{l}$ of $g$ to $M_{l}$ is a pseudo-Riemannian metric on $M_{l}$.

- For a smooth function $v: \mathbb{R}_{>0} \rightarrow \mathbb{R}_{>0}$, there is an isometry between $\left(\mathbb{R}_{>0} \times M_{l}, v(r)\left(\frac{1}{\alpha r} d r^{2}+\frac{r}{l} g_{l}\right)\right)$ and $(M,(v \circ f) g)$.

The more detailed description is given in Theorem 3.3 .

Hence $(M,(v \circ f) g)$ has the structure of a warped product, which is a great advantage. For example, the geodesic equations get complicated under the 
conformal transformations in general, but we can treat them in a simpler way. We can say the same for the sectional curvature and the metric completion.

We summarize results obtained by analyzing the sectional curvature, geodesics, and the metric completion of the warped product pseudo-Riemannian metric (2.15). Here, we use the notation of Theorem 1.2 and $(g, f)$ is a homogeneous pair on a manifold $M$.

(1) When $\operatorname{dim} M=2$, we construct a 2 parameter family of pseudo-Riemannian metrics of constant sectional curvature in the conformal class of $g$ (Corollary 3.17). The same is true when $M$ is a direct product of such manifolds with $\operatorname{dim} \leq 2$ (Remark 3.18).

(2) We construct a 1 parameter family of constant sectional curvature pseudoRiemannian metrics in the conformal class of $g$ if the level set $\left(M_{l}, g_{l}\right)$ has constant sectional curvature (Proposition 3.11). If the sectional curvature of the level set $\left(M_{l}, g_{l}\right)$ is bounded and $g$ is positive or negative definite, we give the bound of the sectional curvature of $(v \circ f) g$ for some $v$ (Corollary 3.15).

For a homogeneous pair $(g, f)$, we define a new pseudo-Riemannian metric $\hat{g}$ in (3.8) such that $(\hat{g}, f)$ is also a homogeneous pair. This pseudoRiemannian metric $\hat{g}$ has a different signature from $g$ and appears in many examples. See Sections 4 and 5. We give further results of this kind for $\hat{g}$ (Corollaries 3.19 and 3.20).

(3) When $v(r)=r^{\beta}$ for $\beta \in \mathbb{R}$, we describe geodesics of $f^{\beta} g$ explicitly using those in $\left(M_{l}, g_{l}\right)$ (Proposition 3.21). Then we give the conditions on $\beta$ so that the function $f$ is convex or concave w.r.t. $f^{\beta} g$ (Proposition 3.22).

(4) When $g$ is positive definite and the pseudometric $d_{g}$ induced from $g$ is a metric, we describe the metric completion of $M$ w.r.t. $(v \circ f) g$ for some of $v$ in terms of the metric completion of $M_{l}$ w.r.t. $g_{l}$ (Theorem 3.23).

Note that to know the above geometric structures completely, we need the information of $\left(M_{l}, g_{l}\right)$, which is obtained from that of $(M, g)$ (Lemma 3.13, Proposition 3.25). However, by the results above, if we have the information of $(M,(v \circ f) g)$ for one $v$, we can obtain the information of $(M,(\tilde{v} \circ f) g)$ for many other $\tilde{v}$ 's.

We can apply results above to many geometric problems. See Section 5 . We list some particularly important results.

(5) We generalize the result of Clarke and Rubinstein (CR2) about the metric completion of the space of Riemannian metrics w.r.t. the conformal deformations of the Ebin metric $g_{E}$ (Theorem [5.11).

They considered the conformal transformations of the form $g_{E} / f^{p}$, where $f$ is the volume functional and $p \in \mathbb{Z}$. We can determine the metric completion w.r.t. $(v \circ f) g_{E}$ for more general functions $v: \mathbb{R}_{>0} \rightarrow \mathbb{R}_{>0}$. In particular, we can give infinitely many examples whose metric completions are strictly smaller than that of $g_{E}$.

(6) There are two canonical Riemannian metrics on the $G_{2}$ moduli space, which are related by the conformal transformation. Both of them are also studied in detail (cf. GY, KLL). We can show that they have different metric completions (Corollary [5.2). 
This paper is organized as follows. In Section 2, we study in detail the geometric structures of a warped product such as the the sectional curvature, the geodesics and the metric completion. In Section 3. we prove Theorem 1.2 (Theorem 3.3) and results (1)-(4) above by the results in Section 2 In Section 4 . we show that some pseudo-Hessian manifolds admit a homogeneous pair, which recovers Loftin, Theorem 1] and [Totaro, Lemmas 2,1 and 2.4]. In Section 5. we give examples as previously stated and apply our method. In Appendix A, we summarize the notations and basic definitions used in this paper.

Acknowledgements: The author would like to thank Sergey Grigorian, Spiro Karigiannis and Burt Totaro for motivating this study. He is grateful to Hitoshi Furuhata for pointing out that the flatness assumption on the connection in Proposition 4.1 is unnecessary. He thanks Takashi Kurose for the useful advice on Hessian geometry. He thanks Sumio Yamada for letting him know the theory of the Teichmüller space. He is indebted to an anonymous referee for the careful reading of an earlier version of this paper and useful comments on it.

This work is supported by JSPS KAKENHI Grant Number JP17K14181 and Research Grants of Yoshishige Abe Memorial Fund.

\section{Warped products}

Let $\left(X, g_{X}\right)$ and $\left(Y, g_{Y}\right)$ be pseudo Riemannian manifolds and $\rho: X \rightarrow \mathbb{R}_{>0}$ be a positive smooth function on $X$. Let $\pi_{X}: X \times Y \rightarrow X$ and $\pi_{Y}: X \times Y \rightarrow Y$ be the canonical projections. The warped product $X \times{ }_{\rho} Y$ is a product manifold $X \times Y$ with the pseudo-Riemannian metric $g=\pi_{X}^{*} g_{X}+\left(\rho \circ \pi_{X}\right)^{2} \pi_{Y}^{*} g_{Y}$ :

$$
X \times_{\rho} Y=\left(X \times Y, g=\pi_{X}^{*} g_{X}+\left(\rho \circ \pi_{X}\right)^{2} \pi_{Y}^{*} g_{Y}\right) .
$$

For simplicity, we drop $\pi_{X}$ and $\pi_{Y}$ and write $X \times{ }_{\rho} Y=\left(X \times Y, g=g_{X}+\rho^{2} g_{Y}\right)$. We study the geometric structures of warped products in detail for the application in Section 3

\subsection{The curvature tensor and the geodesics}

In this subsection, we study the curvature tensor and the geodesics of the warped product $X \times{ }_{\rho} Y=\left(X \times Y, g=g_{X}+\rho^{2} g_{Y}\right)$ based on ['Neill, Section 7].

The vector fields on $X$ and $Y$ are canonically extended to the vector fields on $X \times Y$. We identify these vector fields.

\subsubsection{The curvature tensor}

Lemma 2.1 (Q’Neill, Proposition 7.42]). Use the notation of Appendix A, For vector fields $x, y, z \in \mathfrak{X}(X)$ and $a, b, c \in \mathfrak{X}(Y)$, the curvature tensor $R^{g}$ of $g$ is 
given as follows.

$$
\begin{aligned}
& R^{g}(x, y) z=R^{g_{X}}(x, y) z(\in \mathfrak{X}(X)), \\
& R^{g}(a, x) y=-\frac{\left(\nabla^{g_{X}} d \rho\right)(x, y)}{\rho} a, \\
& R^{g}(x, y) a=R^{g}(a, w) x=0, \\
& R^{g}(x, a) b=-\frac{g(a, b)}{\rho} \nabla_{x}^{g_{X}}\left(\operatorname{grad}^{g_{X}} \rho\right), \\
& R^{g}(a, b) c=R^{g_{Y}}(a, b) c+\frac{g_{X}\left(\operatorname{grad}^{g_{X}} \rho, \operatorname{grad}^{g_{X}} \rho\right)}{\rho^{2}}(g(a, c) b-g(b, c) a) .
\end{aligned}
$$

where $\operatorname{grad}^{g_{X}} \rho \in \mathfrak{X}(X)$ is defined by $g_{X}\left(\operatorname{grad}^{g_{X}} \rho, \cdot\right)=d \rho$.

Note that we adopt the different sign convention of the curvature tensor from ['Neill, Lemma 3.35].

\subsubsection{The geodesics}

Next, we consider the geodesics of the warped product. The geodesic equation is described as follows.

Lemma 2.2 (@'Neill, Proposition 7.38]). Use the notation of Appendix $\AA$. A path $\gamma: J_{1} \ni t \mapsto(r(t), y(t)) \in X \times{ }_{\rho} Y$, where $J_{1} \subset \mathbb{R}$ is an open interval, is a geodesic if and only if

$$
\begin{aligned}
& \nabla_{\frac{d}{d t}}^{r^{*} T X} \dot{r}=g_{Y}(\dot{y}, \dot{y})(\rho \circ r) \cdot\left(\operatorname{grad}^{g_{X}} \rho\right) \circ r, \\
& \nabla_{\frac{d}{d t}}^{y^{*} T Y} \dot{y}=\frac{-2}{\rho \circ r} \frac{d(\rho \circ r)}{d t} \cdot \dot{y},
\end{aligned}
$$

where $\nabla^{r^{*} T X}$ (resp. $\nabla^{y^{*} T Y}$ ) is the induced connection from the Levi-Civita connection of $g_{X}$ (resp. $g_{Y}$ ) along the path $t \mapsto r(t)$ (resp. $t \mapsto y(t)$ ).

Note that (2.2) implies that $t \mapsto y(t)$ is a pregeodesic in $Y$. That is, a reparametrization of $y$ is a geodesic (['Neill, Remark 7.39]).

We rewrite the geodesic equations. We first prove the following.

Lemma 2.3. For any path $\hat{y}: J_{2} \rightarrow Y$ and a smooth map $\theta: J_{1} \rightarrow J_{2}$, where $J_{1}, J_{2} \subset \mathbb{R}$ are open intervals, we have

$$
\nabla_{\frac{d}{d t}}^{(\hat{y} \circ \theta)^{*} T Y} \frac{d(\hat{y} \circ \theta)}{d t}=\frac{d^{2} \theta}{d t^{2}} \cdot\left(\frac{d \hat{y}}{d s} \circ \theta\right)+\left(\frac{d \theta}{d t}\right)^{2} \cdot\left(\nabla_{\frac{d}{d s}}^{\hat{y}^{*} T Y} \frac{d \hat{y}}{d s}\right) \circ \theta,
$$

where $\nabla^{(\hat{y} \circ \theta)^{*} T Y}$ is the induced connection from the Levi-Civita connection of $g_{Y}$ along the path $s \mapsto(\hat{y} \circ \theta)(s)$.

Proof. Since $\frac{d(\hat{y} \circ \theta)}{d t}=\frac{d \theta}{d t} \cdot\left(\frac{d \hat{y}}{d s} \circ \theta\right)$, we have

$$
\nabla_{\frac{d}{d t}}^{(\hat{y} \circ \theta)^{*} T Y} \frac{d(\hat{y} \circ \theta)}{d t}=\frac{d^{2} \theta}{d t^{2}} \cdot\left(\frac{d \hat{y}}{d s} \circ \theta\right)+\frac{d \theta}{d t} \cdot \nabla_{\frac{d}{d t}}^{\theta^{*} \hat{y}^{*} T Y}\left(\frac{d \hat{y}}{d s} \circ \theta\right) .
$$

By the definition of the covariant derivative along the map, we have $\nabla_{\frac{d}{d t}}^{\theta^{*} \hat{y}^{*} T Y}\left(\frac{d \hat{y}}{d s} \circ \theta\right)=$ $\left(\nabla_{\frac{d \theta}{d t}}^{\hat{y}^{*} T Y} \frac{d \hat{y}}{d s}\right) \circ \theta$, which gives the proof. 
Now we rewrite geodesic equations as follows.

Proposition 2.4. The geodesic $\gamma:(-\epsilon, \epsilon) \ni t \mapsto(r(t), y(t)) \in X \times{ }_{\rho} Y$ with the initial position $\left(r_{0}, y_{0}\right) \in X \times Y$ and the initial velocity $\left(\dot{r}_{0}, \dot{y}_{0}\right) \in T_{r_{0}} X \times T_{y_{0}} Y$ is given as follows.

(1) The map $r(t)$ is given by the solution of

$$
\begin{gathered}
\nabla_{\frac{d}{d t}}^{r^{*} T X} \dot{r}=\frac{E_{1}}{(\rho \circ r)^{3}} \cdot\left(\operatorname{grad}^{g_{X}} \rho\right) \circ r, \\
r(0)=r_{0}, \quad \dot{r}(0)=\dot{r}_{0},
\end{gathered}
$$

where $E_{1}=g_{Y}\left(\dot{y}_{0}, \dot{y}_{0}\right)\left(\rho\left(r_{0}\right)\right)^{4}$.

(2) The map $y(t)$ is given by

$$
y(t)=\hat{y}\left(\int_{0}^{t} \frac{E_{3}}{(\rho \circ r(\tau))^{2}} d \tau\right),
$$

where $\hat{y}(s)$ is the geodesic in $\left(Y, g_{Y}\right)$ with the initial position $y_{0} \in Y$ and the initial velocity $\dot{y}_{0} \in T_{y_{0}} Y$, and $E_{3}=\rho\left(r_{0}\right)^{2}$.

Proof. It is easy to see that $(r(t), y(t))$ given above satisfies $(r(0), y(0))=\left(r_{0}, y_{0}\right)$ and $(\dot{r}(0), \dot{y}(0))=\left(\dot{r}_{0}, \dot{y}_{0}\right)$. We show that $(r(t), y(t))$ satisfies (2.1) and (2.2). Setting

$$
\theta(t)=\int_{0}^{t} \frac{E_{3}}{(\rho \circ r(\tau))^{2}} d \tau
$$

we have $y=\hat{y} \circ \theta$. Then since $\dot{y}=\frac{d y}{d t}=\frac{E_{3}}{(\rho \circ r)^{2}} \frac{d \hat{y}}{d s} \circ \theta$, it follows that

$$
g_{Y}(\dot{y}, \dot{y})(\rho \circ r)^{4}=E_{3}^{2} \cdot g_{Y}\left(\frac{d \hat{y}}{d s} \circ \theta, \frac{d \hat{y}}{d s} \circ \theta\right) .
$$

Since $\hat{y}$ is geodesic, $g_{Y}\left(\frac{d \hat{y}}{d s} \circ \theta, \frac{d \hat{y}}{d s} \circ \theta\right)$ is constant. Thus $g_{Y}(\dot{y}, \dot{y})(\rho \circ r)^{4}$ is constant, which is equal to $E_{1}$. Then (2.1) is immediate from (2.3).

Next, we show that $y(t)$ satisfies (2.2). Lemma 2.3 implies that

$$
\nabla_{\frac{d}{d t}}^{y^{*} T Y} \frac{d y}{d t}=\frac{d^{2} \theta}{d t^{2}} \cdot\left(\frac{d \hat{y}}{d s} \circ \theta\right)+\left(\frac{d \theta}{d t}\right)^{2} \cdot\left(\nabla_{\frac{d}{d s}}^{\hat{y}^{*} T Y} \frac{d \hat{y}}{d s}\right) \circ \theta
$$

Since $\hat{y}$ is a geodesic, we have $\nabla_{\frac{d}{d s}}^{\hat{y}^{*} T Y} \frac{d \hat{y}}{d s}=0$. Since $\frac{d y}{d t}=\frac{E_{3}}{(\rho \circ r)^{2}} \frac{d \hat{y}}{d s} \circ \theta$, we have $\frac{d \hat{y}}{d s} \circ \theta=\frac{(\rho \circ r)^{2}}{E_{3}} \frac{d y}{d t}$. We also compute $\frac{d^{2} \theta}{d t^{2}}=\frac{d}{d t}\left(\frac{E_{3}}{(\rho \circ r)^{2}}\right)=\frac{-2 E_{3}}{(\rho \circ r)^{3}} \frac{d(\rho \circ r)}{d t}$. Then these equations imply (2.2).

\subsection{The case $\operatorname{dim} X=1$}

In this subsection, we show more detailed descriptions of the curvature tensor and the geodesic equations when $X$ is 1-dimensional. That is, supposing that $\left(X, g_{X}\right)=\left(I, \xi d r^{2}\right)$, where $I \subset \mathbb{R}$ is an open interval, $r$ is a coordinate on $I$ and $\xi=\xi(r)$ is a nowhere vanishing function on $I$, we consider the warped product

$$
X \times{ }_{\rho} Y=I \times{ }_{\rho} Y=\left(I \times Y, g=\xi d r^{2}+\rho^{2} g_{Y}\right) .
$$




\subsubsection{The curvature tensor}

Lemma 2.5. Use the notation of Appendix A. Set $\partial_{r}=\partial / \partial r$. We have for linearly independent $a, b \in T_{y} Y$ for $y \in Y$

$$
\begin{aligned}
g\left(R^{g}\left(\partial_{r}, a\right) b, \partial_{r}\right) & =g(a, b) \cdot \frac{-2 \rho^{\prime \prime} \xi+\rho^{\prime} \xi^{\prime}}{2 \rho \xi}, \\
K^{g}\left(\partial_{r}, a\right) & =\frac{-2 \rho^{\prime \prime} \xi+\rho^{\prime} \xi^{\prime}}{2 \rho \xi^{2}}, \\
K^{g}(a, b) & =\frac{1}{\rho^{2}}\left(K^{g_{Y}}(a, b)-\frac{\left(\rho^{\prime}\right)^{2}}{\xi}\right) .
\end{aligned}
$$

Note that the second equation is independent of $a \in T_{y} Y$. Hence we define a function $K^{g}\left(\partial_{r}\right)$ on $I$ by

$$
K^{g}\left(\partial_{r}\right)=K^{g}\left(\partial_{r}, a\right)=\frac{-2 \rho^{\prime \prime} \xi+\rho^{\prime} \xi^{\prime}}{2 \rho \xi^{2}} .
$$

Proof. By Lemma 2.1, we compute

$$
\begin{aligned}
g\left(R^{g}\left(\partial_{r}, a\right) b, \partial_{r}\right) & =-\frac{g(a, b)}{\rho} \cdot g\left(\nabla_{\partial_{r}}^{g_{X}} \operatorname{grad}^{g_{X}} \rho, \partial_{r}\right) \\
& =-\frac{g(a, b)}{\rho} \cdot\left(\partial_{r}\left(d \rho\left(\partial_{r}\right)\right)-d \rho\left(\nabla_{\partial_{r}}^{g_{X}} \partial_{r}\right)\right)=\frac{g(a, b)}{\rho} \cdot\left(-\rho^{\prime \prime}+d \rho\left(\nabla_{\partial_{r}}^{g_{X}} \partial_{r}\right)\right) .
\end{aligned}
$$

By the Koszul formula, it follows that

$$
2 g_{X}\left(\nabla_{\partial_{r}}^{g_{X}} \partial_{r}, \partial_{r}\right)=\partial_{r} g_{X}\left(\partial_{r}, \partial_{r}\right)=\xi^{\prime}, \quad \text { and hence } \quad \nabla_{\partial_{r}}^{g_{X}} \partial_{r}=\frac{\xi^{\prime}}{2 \xi} \partial_{r} .
$$

Thus we obtain (2.5). This also implies (2.6).

To prove (2.7), we use Lemma 2.1 again and compute

$$
\begin{aligned}
g\left(R^{g}(a, b) b, a\right) & =g\left(R^{g_{Y}}(a, b) b+\frac{g_{X}\left(\operatorname{grad}^{g_{X}} \rho, \operatorname{grad}^{g_{X}} \rho\right)}{\rho^{2}}(g(a, b) b-g(b, b) a), a\right) \\
& =\rho^{2} g_{Y}\left(R^{g_{Y}}(a, b) b, a\right)+\frac{\left(\rho^{\prime}\right)^{2}}{\rho^{2} \xi}\left(g(a, b)^{2}-g(a, a) g(b, b)\right),
\end{aligned}
$$

where we use $\operatorname{grad}^{g_{X}} \rho=\left(\rho^{\prime} / \xi\right) \partial_{r}$. Using

$$
g(a, a) g(b, b)-g(a, b)^{2}=\rho^{4}\left(g_{Y}(a, a) g_{Y}(b, b)-g_{Y}(a, b)^{2}\right),
$$

we obtain (2.7).

From these formulae, the sectional curvature for general two vectors in $T(I \times$ $Y)$ are computed as follows.

Lemma 2.6. Take any linearly independent $A=k_{1} \partial_{r}+a$ and $B=k_{2} \partial_{r}+b$, where $k_{1}, k_{2} \in \mathbb{R}$ and $a, b \in T_{y} Y$ for $y \in Y$. If $a$ and $b$ are linearly independent, we have

$K^{g}(A, B)=\frac{K^{g}\left(\partial_{r}\right) \xi g\left(k_{2} a-k_{1} b, k_{2} a-k_{1} b\right)+K^{g}(a, b)\left(g(a, a) g(b, b)-g(a, b)^{2}\right)}{\xi g\left(k_{2} a-k_{1} b, k_{2} a-k_{1} b\right)+g(a, a) g(b, b)-g(a, b)^{2}}$.

If $a$ and $b$ are linearly dependent, we have $K^{g}(A, B)=K^{g}\left(\partial_{r}\right)$. 
Thus $K^{g}$ is essentially controlled by $K^{g}\left(\partial_{r}\right)$ and $\left.K^{g}\right|_{T Y \times T Y}$.

Proof. For simplicity, we write $R^{g}\left(A_{1}, A_{2}, A_{3}, A_{4}\right)=g\left(R^{g}\left(A_{1}, A_{2}\right) A_{3}, A_{4}\right)$ for $A_{1}, \cdots, A_{4} \in T(I \times Y)$. Then by Lemma 2.1, we have

$$
R^{g}\left(\partial_{r}, a_{1}, a_{2}, a_{3}\right)=0
$$

for $a_{1}, a_{2}, a_{3} \in T_{y} Y$. Using this, we compute

$$
\begin{aligned}
& R^{g}(A, B, B, A) \\
= & R^{g}\left(A, B, k_{2} \partial_{r}, a\right)+R^{g}\left(A, B, b, k_{1} \partial_{r}\right)+R^{g}(A, B, b, a) \\
= & -2 k_{1} k_{2} R^{g}\left(\partial_{r}, a, b, \partial_{r}\right)+k_{2}^{2} R^{g}\left(a, \partial_{r}, \partial_{r}, a\right)+k_{1}^{2} R^{g}\left(\partial_{r}, b, b, \partial_{r}\right)+R^{g}(a, b, b, a) .
\end{aligned}
$$

By (2.5), it follows that

$$
R^{g}\left(\partial_{r}, a, b, \partial_{r}\right)=\frac{-2 \rho^{\prime \prime} \xi+\rho^{\prime} \xi^{\prime}}{2 \rho \xi} g(a, b)=K^{g}\left(\partial_{r}\right) \xi g(a, b) .
$$

Then we have

$$
R^{g}(A, B, B, A)=K^{g}\left(\partial_{r}\right) \xi g\left(k_{2} a-k_{1} b, k_{2} a-k_{1} b\right)+R^{g}(a, b, b, a) .
$$

On the other hand, we have $g(A, B)=k_{1} k_{2} \xi+g(a, b)$. Hence

$$
\begin{aligned}
& g(A, A) g(B, B)-g(A, B)^{2} \\
= & \left(k_{1}^{2} \xi+g(a, a)\right) \cdot\left(k_{2}^{2} \xi+g(b, b)\right)-\left(k_{1} k_{2} \xi+g(a, b)\right)^{2} \\
= & \xi\left(-2 k_{1} k_{2} g(a, b)+k_{2}^{2} g(a, a)+k_{1}^{2} g(b, b)\right)+g(a, a) g(b, b)-g(a, b)^{2} \\
= & \xi g\left(k_{2} a-k_{1} b, k_{2} a-k_{1} b\right)+g(a, a) g(b, b)-g(a, b)^{2} .
\end{aligned}
$$

Thus since $R^{g}(a, b, b, a)=K^{g}(a, b)\left(g(a, a) g(b, b)-g(a, b)^{2}\right)$ if $a$ and $b$ are linearly independent, the proof is done.

In this setting, we can characterize the warped product with constant sectional curvature as follows. The following statements are obvious from Lemma 2.6

Corollary 2.7. For $C \in \mathbb{R}, K^{g}=C$ if and only if

$$
K^{g}\left(\partial_{r}\right)=\frac{-2 \rho^{\prime \prime} \xi+\rho^{\prime} \xi^{\prime}}{2 \rho \xi^{2}}=C, \quad K^{g}(a, b)=C
$$

for any linearly independent $a, b \in T Y$.

Remark 2.8. We see that $K^{g}=C$ implies that $K^{g_{Y}}$ is constant. In fact, we compute

$$
\frac{d}{d r}\left(C \rho^{2}+\frac{\left(\rho^{\prime}\right)^{2}}{\xi}\right)=2 C \rho \rho^{\prime}+\frac{2 \rho^{\prime} \rho^{\prime \prime}}{\xi}-\frac{\left(\rho^{\prime}\right)^{2} \xi^{\prime}}{\xi^{2}}=2 \rho \rho^{\prime}\left(C-K^{g}\left(\partial_{r}\right)\right)=0 .
$$

Then (2.7) implies that $K^{g_{Y}}$ is constant.

Lemma2.6 also yields the following estimates. Recall that $\xi g\left(k_{2} a-k_{1} b, k_{2} a-\right.$ $\left.k_{1} b\right) \geq 0$ and $g(a, a) g(b, b)-g(a, b)^{2} \geq 0$ when $g=\xi(r) d r^{2}+\rho^{2} g_{Y}$ is definite in the sense of Definition A.1. 
Corollary 2.9. If $g$ is definite in the sense of Definition A.1, we have $\inf _{\substack{r \in I,\{a, b\} \in G r_{2}(T Y)}} \min \left\{K^{g}\left(\partial_{r}\right), K^{g}(a, b)\right\} \leq K^{g} \leq \sup _{\substack{r \in I \\\{a, b\} \in G r_{2}(T Y)}} \max \left\{K^{g}\left(\partial_{r}\right), K^{g}(a, b)\right\}$, where $G_{2}(T Y)$ is the 2-Grassmannian bundle over $Y$ and $\{a, b\}$ stands for the vector subspace spanned by $a, b \in T Y$.

When $Y$ is also 1-dimensional, we can simplify Corollaries 2.7 and 2.9.

Corollary 2.10. Suppose further that $\left(Y, g_{Y}\right)$ is also 1-dimensional. Then $K^{g}=C \in \mathbb{R}$ if and only if

$$
K^{g}\left(\partial_{r}\right)=\frac{-2 \rho^{\prime \prime} \xi+\rho^{\prime} \xi^{\prime}}{2 \rho \xi^{2}}=C .
$$

If $g$ is definite in the sense of Definition A.1, we have

$$
\inf _{r \in I} K^{g}\left(\partial_{r}\right) \leq K^{g} \leq \sup _{r \in I} K^{g}\left(\partial_{r}\right) .
$$

Note that the condition (2.11) is independent of $g_{Y}$.

\subsubsection{The geodesics}

When $X$ is 1-dimensional, (2.3) is described more explicitly as follows.

Lemma 2.11. Use the notation of Proposition 2.4. The equation (2.3) holds if and only if

$$
\ddot{r}+\frac{\xi^{\prime} \circ r}{2 \xi \circ r}(\dot{r})^{2}-\frac{E_{1}}{(\rho \circ r)^{3}} \cdot \frac{\rho^{\prime} \circ r}{\xi \circ r}=0 .
$$

In particular, we have

$$
(\xi \circ r) \cdot(\dot{r})^{2}+\frac{E_{1}}{(\rho \circ r)^{2}}=E_{2},
$$

where $E_{2}=\xi\left(r_{0}\right)\left(\dot{r}_{0}\right)^{2}+g_{Y}\left(\dot{y}_{0}, \dot{y}_{0}\right)\left(\rho\left(r_{0}\right)\right)^{2}$.

Proof. By the identification $\dot{r}=\dot{r} \partial_{r} \circ r$, we compute

$$
\nabla_{\frac{d}{d t}}^{r^{*} T X} \dot{r}=\ddot{r} \partial_{r} \circ r+\dot{r}\left(\nabla_{\dot{r} \partial_{r}}^{g_{X}} \partial_{r}\right) \circ r \stackrel{\sqrt{2.9}}{=}\left(\ddot{r}+\frac{\xi^{\prime} \circ r}{2 \xi \circ r}(\dot{r})^{2}\right) \partial_{r} \circ r .
$$

Then by $\operatorname{grad}^{g_{X}} \rho=\left(\rho^{\prime} / \xi\right) \partial_{r}$, we see that (2.3) is equivalent to (2.12). Multiplying $2(\xi \circ r) \cdot \dot{r}$ on both sides of (2.12), we have

$$
2(\xi \circ r) \cdot \dot{r} \ddot{r}+\left(\xi^{\prime} \circ r\right) \cdot(\dot{r})^{3}-\frac{2 E_{1}}{(\rho \circ r)^{3}} \cdot\left(\rho^{\prime} \circ r\right) \cdot \dot{r}=0 .
$$

Hence

$$
\frac{d}{d t}\left((\xi \circ r) \cdot(\dot{r})^{2}+\frac{E_{1}}{(\rho \circ r)^{2}}\right)=0
$$

which gives the proof.

To solve (2.13), we can use the method of separation of variables. However, it is hard to describe solutions explicitly in general. 


\subsection{The special case of the case $\operatorname{dim} X=1$}

In this subsection, we assume that $I=\mathbb{R}_{>0}$ for simplicity and the pseudoRiemannian metric $g$ is of the form (2.15). This assumption is useful in Section 3. Assuming this, we can solve many of differential equations in previous subsections explicitly and study the sectional curvature, the geodesics and the metric completion in more detail.

In addition, if we set $k=1$ and $w(r)=1$ in (2.15), $g=g(w)$ is a cylindrical pseudo-Riemannian metric. If we set $k=1$ and $w(r)=r^{2}$ in (2.15), $g=g(w)$ is a conical pseudo-Riemannian metric. Thus this assumption also provides a framework for the unified treatment of these geometrically important examples.

\subsubsection{The sectional curvature}

Assuming that the sectional curvature $K^{g_{Y}}$ of $\left(Y, g_{Y}\right)$ is constant, we construct pseudo-Riemannian metrics of constant sectional curvature. We can apply this in Section 3.2. We begin by the following definition.

Definition 2.12. Set

$$
\begin{aligned}
& \Delta_{1}=\left\{\left(s, C_{1}, C_{2}\right) \in \mathbb{R}^{3} \mid s>0, C_{1}>0, C_{2} \in \mathbb{R}\right\}, \\
& \Delta_{2}=\left\{\left(s, C_{1}, C_{2}\right) \in \mathbb{R}^{3} \mid s=0, C_{1} \geq 0, C_{2} \in \mathbb{R}\right\}, \\
& \Delta_{3}=\left\{\left(s, C_{1}, C_{2}\right) \in \mathbb{R}^{3} \mid s<0, C_{1} \leq 0, C_{2} \in \mathbb{R}\right\} .
\end{aligned}
$$

Then define a function $w\left(s, C_{1}, C_{2}, r\right)$ for $\left(s, C_{1}, C_{2}\right) \in \Delta_{1} \cup \Delta_{2} \cup \Delta_{3}$ and (generic) $r \in \mathbb{R}_{>0}$ as follows.

- $\operatorname{For}\left(s, C_{1}, C_{2}\right) \in \Delta_{1}$, set

$$
w\left(s, C_{1}, C_{2}, r\right)=\frac{C_{1}}{s \cdot\left(\cosh \left(\sqrt{C_{1}}\left(\log r+C_{2}\right)\right)\right)^{2}} .
$$

- $\operatorname{For}\left(0, C_{1}, C_{2}\right) \in \Delta_{2}$, set

$$
w\left(0, C_{1}, C_{2}, r\right)=e^{C_{2}} r^{ \pm 2 \sqrt{C_{1}}} .
$$

- $\operatorname{For}\left(s, C_{1}, C_{2}\right) \in \Delta_{3}$ with $C_{1}<0$, set

$$
w\left(s, C_{1}, C_{2}, r\right)=\frac{C_{1}}{s \cdot\left(\sin \left(\sqrt{-C_{1}}\left(\log r+C_{2}\right)\right)\right)^{2}}
$$

For $\left(s, 0, C_{2}\right) \in \Delta_{3}$, set

$$
w\left(s, 0, C_{2}, r\right)=\frac{-1}{s \cdot\left(\log r+C_{2}\right)^{2}}\left(=\lim _{C_{1} \rightarrow-0} w\left(s, C_{1}, C_{2}, r\right)\right) .
$$

Proposition 2.13. Let $\left(Y, g_{Y}\right)$ be a pseudo Riemannian manifold. Fix $k \in$ $\mathbb{R}-\{0\}$. For a smooth function $w: \mathbb{R}_{>0} \rightarrow \mathbb{R}_{>0}$, define a pseudo-Riemannian metric $g=g(w)$ on $\mathbb{R}_{>0} \times Y$ by

$$
g=g(w)=\frac{k w(r)}{r^{2}} d r^{2}+w(r) g_{Y} .
$$


Defining functions $\xi, \rho: \mathbb{R}_{>0} \rightarrow \mathbb{R}_{>0}$ by

$$
\xi(r)=\frac{k w(r)}{r^{2}}, \quad \rho(r)=\sqrt{w(r)},
$$

we have the following.

(1) Recall (2.8). Given $C \in \mathbb{R}$, the differential equation

$$
K^{g(w)}\left(\partial_{r}\right)=\frac{-2 \rho^{\prime \prime} \xi+\rho^{\prime} \xi^{\prime}}{2 \rho \xi^{2}}=C
$$

w.r.t. $w(r)$ has a 2 parameter family of solutions given by $w(r)=w\left(k C, C_{1}, C_{2}, r\right)$ for $\left(C_{1}, C_{2}\right) \in \mathbb{R}^{2}$ such that $\left(k C, C_{1}, C_{2}\right) \in \Delta_{1} \cup \Delta_{2} \cup \Delta_{3}$, where we use the notation in Definition 2.12.

(2) For $g=g\left(w\left(k C, C_{1}, C_{2}, \cdot\right)\right)$, we have

$$
K^{g}(a, b)=\frac{1}{w(r)}\left(K^{g_{Y}}(a, b)-\frac{C_{1}}{k}\right)+C
$$

for linearly independent $a, b \in T Y$.

(3) The pseudo-Riemannian metric $g=g\left(w\left(k C, C_{1}, C_{2}, \cdot\right)\right)$ has constant sectional curvature $C$ if and only if $g_{Y}$ has constant sectional curvature $C_{1} / k$.

Remark 2.14. By fixing $\left(k C, C_{1}, C_{2}\right)$, the function $w\left(k C, C_{1}, C_{2}, r\right)$ of $r$ is defined for all $r>0$ when $k C \geq 0$. When $k C<0$, it is only defined on the complement of the discrete set of $\mathbb{R}_{>0}$.

Proof. Setting $w(r)=e^{2 W(r)}$ for a smooth function $W: \mathbb{R}_{>0} \rightarrow \mathbb{R}$, we have

$$
\xi=\frac{k e^{2 W}}{r^{2}}, \quad \rho=e^{W} .
$$

Then

$$
\begin{aligned}
-2 \rho^{\prime \prime} \xi+\rho^{\prime} \xi^{\prime} & =k\left(-2\left(W^{\prime \prime}+\left(W^{\prime}\right)^{2}\right) e^{W} \cdot \frac{e^{2 W}}{r^{2}}+W^{\prime} e^{W}\left(\frac{2 W^{\prime} e^{2 W}}{r^{2}}-\frac{2 e^{2 W}}{r^{3}}\right)\right) \\
& =k e^{3 W}\left(-\frac{2 W^{\prime \prime}}{r^{2}}-\frac{2 W^{\prime}}{r^{3}}\right) \\
& =\frac{-2 k e^{3 W}}{r^{3}} \frac{d}{d r}\left(r W^{\prime}\right) .
\end{aligned}
$$

Since $2 \rho \xi^{2}=2 k^{2} e^{5 W} / r^{4}$, we obtain

$$
K^{g(w)}\left(\partial_{r}\right)=\frac{-2 \rho^{\prime \prime} \xi+\rho^{\prime} \xi^{\prime}}{2 \rho \xi^{2}}=-\frac{r}{k e^{2 W}} \frac{d}{d r}\left(r W^{\prime}\right) .
$$

Thus $K^{g(w)}\left(\partial_{r}\right)=C$ is equivalent to

$$
r \frac{d}{d r}\left(r W^{\prime}\right)=-k C e^{2 W}
$$

Multiplying $W^{\prime}$ on both sides, we have

$$
\frac{d}{d r}\left(\left(r W^{\prime}\right)^{2}\right)=-k C \frac{d}{d r} e^{2 W} .
$$


Thus we obtain

$$
\left(r W^{\prime}\right)^{2}=-k C e^{2 W}+C_{1}
$$

for $C_{1} \in \mathbb{R}$. This can be solved by the method of separation of variables. After a straightforward computation, we obtain the following.

- When $k C>0$, we have

$$
W(r)=-\log \left(\sqrt{\frac{k C}{C_{1}}} \cosh \left(\sqrt{C_{1}}\left(\log r+C_{2}\right)\right)\right) \quad\left(C_{1}>0, C_{2} \in \mathbb{R}\right) .
$$

- When $C=0$, we have

$$
W(r)= \pm \sqrt{C_{1}} \cdot \log r+\frac{C_{2}}{2} \quad\left(C_{1} \geq 0, C_{2} \in \mathbb{R}\right),
$$

- When $k C<0$, we have

$$
W(r)=-\log \left|\sqrt{-k C}\left(\log r+C_{2}\right)\right| \quad\left(C_{2} \in \mathbb{R}\right),
$$

which corresponds to $C_{1}=0$, or

$$
W(r)=-\log \left|\sqrt{\frac{k C}{C_{1}}} \sin \left(\sqrt{-C_{1}}\left(\log r+C_{2}\right)\right)\right| \quad\left(C_{1}<0, C_{2} \in \mathbb{R}\right) .
$$

Then we obtain (1) via $w(r)=e^{2 W(r)}$. For the proof of (2), recall by (2.7) that $K^{g}(a, b)=\frac{1}{\rho^{2}}\left(K^{g_{Y}}(a, b)-\frac{\left(\rho^{\prime}\right)^{2}}{\xi}\right)$. Then we compute

$$
-\frac{\left(\rho^{\prime}\right)^{2}}{\xi}=-\frac{\left(W^{\prime}\right)^{2} e^{2 W} \cdot r^{2}}{k e^{2 W}} \stackrel{2.17}{=} \frac{1}{k}\left(-C_{1}+k C e^{2 W}\right)=-\frac{C_{1}}{k}+C \rho^{2},
$$

which gives the proof of (2). The statement (3) is immediate from (1), (2) and Corollary 2.7

Remark 2.15. For a function $w_{1}: \mathbb{R}_{>0} \rightarrow \mathbb{R}_{>0}$, define a function $w_{2}: \mathbb{R}_{>0} \rightarrow$ $\mathbb{R}_{>0}$ by $w_{2}(r)=w_{1}(1 / r)$. Then $\left(\mathbb{R}_{>0} \times Y, g\left(w_{1}\right)\right)$ and $\left(\mathbb{R}_{>0} \times Y, g\left(w_{2}\right)\right)$ are isometric via $(r, y) \mapsto(1 / r, y)$.

This is because $d r^{2} / r^{2}=(d \log r)^{2}$ is invariant under $r \mapsto 1 / r$. In particular, the space of solutions $w$ of $K^{g(w)}\left(\partial_{r}\right)=C$ given in Proposition 2.13 (1) is invariant under $w(r) \mapsto w(1 / r)$.

We have the following sectional curvature bound by Corollary 2.9 and Proposition 2.13

Corollary 2.16. Use the notation of Definition 2.10 and Proposition 2.13. Suppose that $g=g\left(w\left(k C, C_{1}, C_{2}, \cdot\right)\right)$, where $\left(k C, C_{1}, C_{2}\right) \in \Delta_{1} \cup \Delta_{2} \cup \Delta_{3}$, is definite in the sense of Definition A.1. Then we have

- $K^{g} \geq C$ when $K^{g_{Y}} \geq C_{1} / k$, and

- $K^{g} \leq C$ when $K^{g_{Y}} \leq C_{1} / k$. 
Furthermore, $K^{g}=C$ if and only if $K^{g_{Y}}=C_{1} / k$.

When $\operatorname{dim} Y=1$, we do not need the assumption on $K^{g_{Y}}$ by Corollary 2.10, Then Proposition 2.13 (1) implies the following.

Corollary 2.17. Use the notation of Definition 2.12 and Proposition 2.13] In addition to the assumptions of Proposition 2.13, suppose further that $\operatorname{dim} Y=$ 1. Then given $C \in \mathbb{R}, g=g(w)$ has constant sectional curvature $C$ if $w=$ $w\left(k C, C_{1}, C_{2}, \cdot\right)$, where $C_{1}, C_{2} \in \mathbb{R}$ such that $\left(k C, C_{1}, C_{2}\right) \in \Delta_{1} \cup \Delta_{2} \cup \Delta_{3}$.

\subsubsection{The geodesics}

By Proposition 2.4 and Lemma 2.11 we now describe the geodesics explicitly for $g\left(w\left(0, C_{1}, C_{2}, \cdot\right)\right)$ for $C_{1} \geq 0$ and $C_{2} \in \mathbb{R}$. (We tried to describe the geodesics explicitly for $g\left(w\left(s, C_{1}, C_{2}, \cdot\right)\right)$ for any $s$, but we could do it only when $s=0$. )

Setting $w(r)=r^{C_{0}}$ for $C_{0} \in \mathbb{R}$, we consider the geodesics for the pseudoRiemannian metric

$$
g=g\left(r^{C_{0}}\right)=k r^{C_{0}-2} d r^{2}+r^{C_{0}} g_{Y} .
$$

Note that since $g(\lambda w)=\lambda g(w)$ for $\lambda>0$ and $w: \mathbb{R}_{>0} \rightarrow \mathbb{R}_{>0}$, and the Levi-Civita connection is invariant under the scalar multiplication of a pseudoRiemannian metric, we may assume that the coefficient of $r^{C_{0}}$ is 1 .

Proposition 2.18. Use the notation of Definition 2.12 and Proposition 2.13 The geodesic $\gamma:(-\epsilon, \epsilon) \ni t \mapsto(r(t), y(t)) \in \mathbb{R}_{>0} \times Y$ with the initial position $\left(r_{0}, y_{0}\right) \in \mathbb{R}_{>0} \times Y$ and the initial velocity $\left(\dot{r}_{0}, \dot{y}_{0}\right) \in \mathbb{R} \times T_{y_{0}} Y$ w.r.t. $g=g\left(r^{C_{0}}\right)$ is given as follows.

(1) When $C_{0} \neq 0$,

$$
\begin{aligned}
& r(t)=r_{0}\left(1+C_{0} \frac{\dot{r}_{0}}{r_{0}} t+C_{0}^{2} F t^{2}\right)^{\frac{1}{C_{0}}}, \\
& y(t)=\hat{y}\left(\int_{0}^{t} \frac{d \tau}{1+C_{0} \frac{\dot{r}_{0}}{r_{0}} \tau+C_{0}^{2} F \tau^{2}}\right),
\end{aligned}
$$

where $F=\frac{1}{4}\left(\left(\frac{\dot{r}_{0}}{r_{0}}\right)^{2}+\frac{g_{Y}\left(\dot{y}_{0}, \dot{y}_{0}\right)}{k}\right)$ and $\hat{y}(s)$ is the geodesic in $\left(Y, g_{Y}\right)$ with the initial position $y_{0} \in Y$ and the initial velocity $\dot{y}_{0} \in T_{y_{0}} Y$.

(2) When $C_{0}=0$,

$$
r(t)=r_{0} e^{\frac{\dot{r}_{0}}{r_{0}} t}, \quad y(t)=\hat{y}(t) .
$$

Remark 2.19. By a straightforward computation, the integral $\int_{0}^{t} \frac{d \tau}{1+C_{0} \frac{r_{0}}{r_{0}} \tau+C_{0}^{2} F \tau^{2}}$ 
in (2.19) can be explicitly computed as follows.

$$
\begin{cases}\frac{2}{C_{0}} \sqrt{\frac{k}{g_{Y}\left(\dot{y}_{0}, \dot{y}_{0}\right)}} \arctan \left(\frac{\sqrt{\frac{g_{Y}\left(\dot{y}_{0}, \dot{y}_{0}\right)}{k}}}{\frac{r_{0}}{r_{0}} t+\frac{2}{C_{0}}}\right) & \text { if } \quad F \neq 0, k g_{Y}\left(\dot{y}_{0}, \dot{y}_{0}\right)>0, \\ \frac{4 r_{0}^{2} F t}{\dot{r}_{0}\left(2 r_{0} C_{0} F t+\dot{r}_{0}\right)} & \text { if } \quad F \neq 0, k g_{Y}\left(\dot{y}_{0}, \dot{y}_{0}\right)=0, \\ \frac{2}{C_{0}} \sqrt{\frac{-k}{g_{Y}\left(\dot{y}_{0}, \dot{y}_{0}\right)}} \operatorname{arctanh}\left(\frac{\sqrt{\frac{-g_{Y}\left(\dot{y}_{0}, \dot{y}_{0}\right)}{k}} t}{\frac{r_{0}}{r_{0}} t+\frac{2}{C_{0}}}\right) & \text { if } \quad F \neq 0, k g_{Y}\left(\dot{y}_{0}, \dot{y}_{0}\right)<0, \\ \frac{r_{0}}{C_{0} \dot{r}_{0}} \log \left(1+C_{0} \frac{\dot{r}_{0}}{r_{0}} t\right) & \text { if } \quad F=0, \dot{r}_{0} \neq 0, \\ t & \text { if } \quad F=0, \dot{r}_{0}=0 .\end{cases}
$$

These formulae implies that geodesics are not defined for all $t \in \mathbb{R}$ in general. In particular, $g\left(r^{C_{0}}\right)$ in (2.18) is incomplete if $C_{0} \neq 0$. It is complete if $C_{0}=0$ and $g_{Y}$ is complete. This is consistent with Theorem 2.27.

Proof. Setting

$$
\xi(r)=k r^{C_{0}-2}, \quad \rho(r)=r^{C_{0} / 2},
$$

we have to solve (2.12) and compute (2.4) by Proposition 2.4 and Lemma 2.11. By Lemma 2.11 we first solve (2.13). It is equivalent to

$$
k r^{2 C_{0}-2}(\dot{r})^{2}+E_{1}=E_{2} r^{C_{0}} .
$$

Now suppose that $C_{0}=0$. Then (2.21) implies that $r^{-2}(\dot{r})^{2}$ is constant, and hence $r(t)=L_{1} e^{t L_{2}}$ for $L_{1}, L_{2} \in \mathbb{R}$. Since $r(0)=r_{0}$ and $\dot{r}(0)=\dot{r}_{0}$, we have $r(t)=r_{0} e^{t \dot{r}_{0} / r_{0}}$. It is straightforward to see that this satisfies (2.12). Since $(\rho(r))^{2}=1$, (2.4) implies that $y(t)=\hat{y}(t)$.

Next, suppose that $C_{0} \neq 0$. Setting $s(t)=(r(t))^{C_{0}}$, we have $\dot{s}=C_{0} r^{C_{0}-1} \dot{r}$. Then (2.21) becomes

$$
\frac{k}{C_{0}^{2}}(\dot{s})^{2}+E_{1}=E_{2} s
$$

Differentiating this equation, we have

$$
\dot{s}\left(\frac{2 k}{C_{0}^{2}} \ddot{s}-E_{2}\right)=0,
$$

which implies that $s(t)=F_{0}+F_{1} t+\frac{C_{0}^{2} E_{2}}{4 k} t^{2}$ for $F_{0}, F_{1} \in \mathbb{R}$ or $s(t)$ is constant. When $s(t)=F_{0}+F_{1} t+\frac{C_{0}^{2} E_{2}}{4 k} t^{2}$, since $r(0)=r_{0}$ and $\dot{r}(0)=\dot{r}_{0}$, we have $F_{0}=r_{0}^{C_{0}}$ and $F_{1}=C_{0} r_{0}^{C_{0}-1} \dot{r}_{0}$. Since

$\frac{C_{0}^{2} E_{2}}{4 k}=\frac{C_{0}^{2}}{4 k}\left(\xi\left(r_{0}\right)\left(\dot{r}_{0}\right)^{2}+g_{Y}\left(\dot{y}_{0}, \dot{y}_{0}\right)\left(\rho\left(r_{0}\right)\right)^{2}\right)=C_{0}^{2} r_{0}^{C_{0}} \cdot \frac{1}{4}\left(\left(\frac{\dot{r}_{0}}{r_{0}}\right)^{2}+\frac{g_{Y}\left(\dot{y}_{0}, \dot{y}_{0}\right)}{k}\right)$,

we see that $r(t)=(s(t))^{1 / C_{0}}$ is given by the first equation of (2.19). It is straightforward to see that this satisfies (2.12).

When $s(t)$ is constant, and hence $r(t)$ is constant, (2.12) implies that $E_{1}=0$. Then (2.21) implies that $E_{2}=0$. By the definitions of $E_{1}$ and $E_{2}$ in Proposition 2.4 and Lemma 2.11, it follows that $g\left(\dot{y}_{0}, \dot{y}_{0}\right)=0$ and $\dot{r}_{0}=0$. Hence this case is reduced to (2.19).

Since $(\rho \circ r(t))^{2}=r(t)^{C_{0}}=s(t)$, (2.4) implies the second equation of (2.19). 
The next corollary is used to prove Proposition 3.22

Corollary 2.20. Let $\gamma:(-\epsilon, \epsilon) \ni t \mapsto(r(t), y(t)) \in \mathbb{R}_{>0} \times Y$ be a geodesic w.r.t. the pseudo-Riemannian metric $g\left(r^{C_{0}}\right)$ in (2.18).

(1) The function $r(t)$ is a convex function if one of the following conditions holds.

- $C_{0}=0$,

- $0<C_{0} \leq 2$ and $k g_{Y}$ is positive definite,

- $C_{0}<0$ and $\mathrm{kg}_{Y}$ is negative definite,

(2) The function $r(t)$ is a concave function if $C_{0} \geq 2$ and $k g_{Y}$ is negative definite.

Proof. By Proposition 2.18, it is obvious that $r(t)$ is convex when $C_{0}=0$. Suppose that $C_{0} \neq 0$. A straightforward calculation gives that

$$
\frac{d^{2} r(t)}{d t^{2}}=\frac{r_{0}}{C_{0}} \cdot\left(1+C_{0} \frac{\dot{r}_{0}}{r_{0}} t+C_{0}^{2} F t^{2}\right)^{\frac{1}{C_{0}}-2} p(t)
$$

where $p(t)$ is a polynomial given by

$$
p(t)=\left(4-2 C_{0}\right)\left(C_{0}^{3} F^{2} t^{2}+C_{0}^{2} \frac{\dot{r}_{0}}{r_{0}} F t\right)+\left(\left(1-C_{0}\right) C_{0}\left(\frac{\dot{r}_{0}}{r_{0}}\right)^{2}+2 C_{0}^{2} F\right) .
$$

When $C_{0}=2$, we have

$$
p(t)=-2\left(\frac{\dot{r}_{0}}{r_{0}}\right)^{2}+8 F=\frac{2 g_{Y}\left(\dot{y}_{0}, \dot{y}_{0}\right)}{k},
$$

which gives the statement for $C_{0}=2$.

Suppose that $C_{0} \neq 0,2$. It is also straightforward to see that the discriminant $\operatorname{disc}(p(t))$ of the quadratic $p(t)$ is given by

$$
\operatorname{disc}(p(t))=4\left(C_{0}-2\right) C_{0}^{3} F^{2} \cdot \frac{g_{Y}\left(\dot{y}_{0}, \dot{y}_{0}\right)}{k} .
$$

Since $r(t)$ is convex (resp. concave) if $4-2 C_{0}>0$ (resp. $4-2 C_{0}<0$ ) and $\operatorname{disc}(p(t)) \leq 0$, we obtain the statement.

Remark 2.21. Use the notation of Definition A.1. By the definition of $g=$ $g\left(r^{C_{0}}\right)$ in (2.18), we can rephrase the conditions in Corollary 2.20 as follows.

- The pseudo-Riemannian metric $\mathrm{kg}_{Y}$ is positive definite if and only if $g$ and $g_{Y}$ are definite.

- The pseudo-Riemannian metric $k g_{Y}$ is negative definite if and only if $g$ is Lorentzian and $g_{Y}$ is definite. 


\subsubsection{The metric completion}

In this subsection, we consider the pseudo-Riemannian metric $g=g(w)$ given in (2.15) again. We assume the following.

- The pseudo-Riemannian metric $g=g(w)$ given in (2.15) is positive definite. That is, $k>0$ and $g_{Y}$ is positive definite.

- The pseudometric $d_{g}$ induced from $g=g(w)$ is a metric. (This is always true when $Y$ is finite dimensional. In the infinite dimensional case, there are examples of a Riemannian metric whose induced pseudometric is identically zero (MM). Note that Lemmas 2.22 and 2.24 (1) imply that $d_{g}$ is a metric if the pseudometric $d_{g_{Y}}$ induced from $g_{Y}$ is a metric. )

We study the metric completion of $\mathbb{R}_{>0} \times Y$ w.r.t. $d_{g}$ following [CR2, Section 5]. Recall that the metric $d_{g}$ between $\left(r_{0}, y_{0}\right)$ and $\left(r_{1}, y_{1}\right) \in \mathbb{R}_{>0} \times Y$ is given by

$$
\begin{aligned}
& d_{g}\left(\left(r_{0}, y_{0}\right),\left(r_{1}, y_{1}\right)\right) \\
= & \inf \left\{\begin{array}{l|l}
L_{g}(c) \mid \begin{array}{l}
c=(r \circ c, y \circ c):[0,1] \rightarrow \mathbb{R}_{>0} \times Y \text { is a piecewise smooth path } \\
\text { with } c(0)=\left(r_{0}, y_{0}\right) \text { and } c(1)=\left(r_{1}, y_{1}\right)
\end{array}
\end{array}\right\},
\end{aligned}
$$

where

$$
\begin{aligned}
L_{g}(c) & =\int_{0}^{1}\left|\frac{d c}{d t}(t)\right|_{g} d t \\
& =\int_{0}^{1} \sqrt{\frac{k w((r \circ c)(t))}{((r \circ c)(t))^{2}}\left(\frac{d(r \circ c)}{d t}(t)\right)^{2}+w((r \circ c)(t))\left|\frac{d(y \circ c)}{d t}(t)\right|_{g_{Y}}^{2}} d t .
\end{aligned}
$$

Here, we use the notation of Appendix \$ Similarly, we can define the metric $d_{g_{Y}}$ induced from $g_{Y}$.

To study the metric completion, we first prove the following lemmas. Fixing $R_{0} \in \mathbb{R}_{>0}$, define a strictly increasing function $T: \mathbb{R}_{>0} \rightarrow \mathbb{R}$ by

$$
T(r)=\int_{R_{0}}^{r} \frac{\sqrt{k w(q)}}{q} d q .
$$

Lemma 2.22. For any $\left(r_{0}, y_{0}\right),\left(r_{1}, y_{1}\right) \in \mathbb{R}_{>0} \times Y$, we have

$$
d_{g}\left(\left(r_{0}, y_{0}\right),\left(r_{1}, y_{1}\right)\right) \geq\left|T\left(r_{1}\right)-T\left(r_{0}\right)\right| .
$$

In particular, $\mathbb{R}_{>0} \times Y \ni(r, y) \mapsto r \in \mathbb{R}_{>0}$ is continuous w.r.t. $d_{g}$.

Proof. Let $c=(r \circ c, y \circ c):[0,1] \rightarrow \mathbb{R}_{>0} \times Y$ be a piecewise smooth path with $c(0)=\left(r_{0}, y_{0}\right)$ and $c(1)=\left(r_{1}, y_{1}\right)$. By (2.22), we compute

$$
L_{g}(c) \geq \int_{0}^{1} \frac{\sqrt{k w(r \circ c)}}{r \circ c}\left|\frac{d}{d t}(r \circ c)\right| d t \geq\left|\int_{0}^{1} \frac{\sqrt{k w(r \circ c)}}{r \circ c} \frac{d}{d t}(r \circ c) d t\right|=\left|T\left(r_{1}\right)-T\left(r_{0}\right)\right| .
$$


As $T$ is strictly increasing, it converges in $\mathbb{R} \cup\{-\infty\}$ (resp. $\mathbb{R} \cup\{\infty\}$ ) as $r \rightarrow 0$ (resp. $r \rightarrow \infty$ ). Set

$$
T_{0}=\lim _{r \rightarrow 0} T(r) \in \mathbb{R} \cup\{-\infty\}, \quad T_{\infty}=\lim _{r \rightarrow \infty} T(r) \in \mathbb{R} \cup\{\infty\} .
$$

Then the following is immediate from Lemma 2.22. This is useful to study the metric completion w.r.t. $d_{g}$.

Corollary 2.23. If $\left\{\left(r_{k}, y_{k}\right)\right\} \subset \mathbb{R}_{>0} \times Y$ is a $d_{g}$-Cauchy sequence, $\left\{r_{k}\right\}$ converges in

$$
\left\{\begin{array}{lll}
\mathbb{R}_{>0} & \text { if } T_{0}=-\infty, & T_{\infty}=\infty, \\
\{0\} \cup \mathbb{R}_{>0} & \text { if } T_{0} \in \mathbb{R}, & T_{\infty}=\infty, \\
\mathbb{R}_{>0} \cup\{\infty\} & \text { if } T_{0}=-\infty, & T_{\infty} \in \mathbb{R}, \\
\{0\} \cup \mathbb{R}_{>0} \cup\{\infty\} & \text { if } T_{0} \in \mathbb{R}, & T_{\infty} \in \mathbb{R} .
\end{array}\right.
$$

Lemma 2.24. Fix $0<R_{1}<R_{2}$. There exist $\delta=\delta\left(R_{1}, R_{2}, T\right)$, a constant depending on $R_{1}, R_{2}$ and $T$, and $C^{\prime}=C^{\prime}\left(R_{1}, R_{2}, w\right), C^{\prime \prime}=C^{\prime \prime}\left(R_{1}, R_{2}, w\right)>$ 0 , constants depending on $R_{1}, R_{2}$ and $w$, such that for any $\left(r_{0}, y_{0}\right),\left(r_{1}, y_{1}\right) \in$ $\left(R_{1}, R_{2}\right) \times Y$

(1) $d_{g}\left(\left(r_{0}, y_{0}\right),\left(r_{1}, y_{1}\right)\right)<\delta \Rightarrow d_{g_{Y}}\left(y_{0}, y_{1}\right) \leq C^{\prime} d_{g}\left(\left(r_{0}, y_{0}\right),\left(r_{1}, y_{1}\right)\right)$.

(2) $d_{g}\left(\left(r_{0}, y_{0}\right),\left(r_{1}, y_{1}\right)\right) \leq\left|T\left(r_{0}\right)-T\left(r_{1}\right)\right|+C^{\prime \prime} d_{g_{Y}}\left(y, y^{\prime}\right)$.

Proof. First, we show that there exists $\delta=\delta\left(R_{1}, R_{2}, T\right)>0$ such that for any $(r, y) \in\left(R_{1}, R_{2}\right) \times Y$ and $\left(r^{\prime}, y^{\prime}\right) \in \mathbb{R}_{>0} \times Y$

$$
d_{g}\left((r, y),\left(r^{\prime}, y^{\prime}\right)\right)<2 \delta \Rightarrow \frac{R_{1}}{2}<r^{\prime}<2 R_{2}
$$

By Lemma 2.22 the map $\mathbb{R}_{>0} \times Y \ni(r, y) \mapsto T(r) \in \mathbb{R}$ is uniformly continuous w.r.t. $d_{g}$. Then for $\epsilon=\min \left\{T\left(R_{1}\right)-T\left(R_{1} / 2\right), T\left(2 R_{2}\right)-T\left(R_{2}\right)\right\}>0$, there exists $\delta=\delta\left(R_{1}, R_{2}, T\right)>0$ such that for any $(r, y),\left(r^{\prime}, y^{\prime}\right) \in \mathbb{R}_{>0} \times Y$

$$
d_{g}\left((r, y),\left(r^{\prime}, y^{\prime}\right)\right)<2 \delta \Rightarrow\left|T(r)-T\left(r^{\prime}\right)\right|<\epsilon .
$$

In particular, if $(r, y) \in\left(R_{1}, R_{2}\right) \times Y$, we see that

$$
T\left(\frac{R_{1}}{2}\right) \leq T\left(R_{1}\right)-\epsilon<T(r)-\epsilon<T\left(r^{\prime}\right)<T(r)+\epsilon<T\left(R_{2}\right)+\epsilon \leq T\left(2 R_{2}\right)
$$

hence we obtain (2.24).

Now we prove (1). Suppose that $d_{g}\left(\left(r_{0}, y_{0}\right),\left(r_{1}, y_{1}\right)\right)<\delta$ for $\delta$ given above. For any $0<\epsilon<\delta$, take a piecewise smooth path $\{c(t)\}_{t \in[0,1]}$ connecting $\left(r_{0}, y_{0}\right)$ and $\left(r_{1}, y_{1}\right)$ such that $L_{g}(c)<d_{g}\left(\left(r_{0}, y_{0}\right),\left(r_{1}, y_{1}\right)\right)+\epsilon$. Then for any $t \in[0,1]$, we have

$$
d_{g}\left(\left(r_{0}, y_{0}\right), c(t)\right) \leq L_{g}\left(\left.c\right|_{[0, t]}\right) \leq L_{g}(c)<\delta+\epsilon<2 \delta
$$

Hence by (2.24), it follows that $\frac{R_{1}}{2}<(r \circ c)(t)<2 R_{2}$ for any $t \in[0,1]$. Thus setting $1 / C^{\prime}=\min \left\{\sqrt{w(r)} \mid r \in\left[\frac{R_{1}}{2}, 2 R_{2}\right]\right\}$, we obtain by (2.22)

$$
L_{g}(c) \geq \int_{0}^{1} \sqrt{w((r \circ c)(t))}\left|\frac{d(y \circ c)}{d t}(t)\right|_{g_{Y}} d t \geq \frac{L_{g_{Y}}(y \circ c)}{C^{\prime}} \geq \frac{d_{g_{Y}}\left(y_{0}, y_{1}\right)}{C^{\prime}}
$$


Since $L_{g}(c)<d_{g}\left(\left(r_{0}, y_{0}\right),\left(r_{1}, y_{1}\right)\right)+\epsilon$ and $\epsilon$ is arbitrarily small, we obtain (1).

Next, we prove (2). Define a path $c:[0,1] \rightarrow \mathbb{R}_{>0} \times Y$ by $c(t)=\left(\left(r_{1}-r_{0}\right) t+r_{0}, \tilde{y}(t)\right)$, where $\tilde{y}:[0,1] \rightarrow Y$ is a path such that $\tilde{y}(0)=y_{0}$ and $\tilde{y}(1)=y_{1}$. Then by (2.22), we see that

$$
d_{g}\left(\left(r_{0}, y_{0}\right),\left(r_{1}, y_{1}\right)\right) \leq L(c) \leq I_{1}+I_{2}
$$

where

$I_{1}=\int_{0}^{1} \frac{\sqrt{k w(r \circ c)}}{r \circ c}\left|r_{1}-r_{0}\right| d t=\left|\int_{0}^{1} \frac{\sqrt{k w(r \circ c)}}{r \circ c}\left(r_{1}-r_{0}\right) d t\right|=\left|T\left(r_{1}\right)-T\left(r_{0}\right)\right|$,

$I_{2}=\int_{0}^{1} \sqrt{w(r \circ c)}\left|\frac{d \tilde{y}}{d t}(t)\right|_{g_{Y}} d t$.

Set $C^{\prime \prime}=\max \left\{\sqrt{w(r)} \mid r \in\left[R_{1}, R_{2}\right]\right\}$. Since $(r \circ c)(t)=\left(r_{1}-r_{0}\right) t+r_{0} \in\left(R_{1}, R_{2}\right)$ for any $t \in[0,1]$, we see that

$$
I_{2} \leq C^{\prime \prime} L_{g_{Y}}(\tilde{y}) .
$$

Since $\tilde{y}$ is arbitrary, we obtain (2).

For a subset $S \subset \mathbb{R}_{>0} \times Y$, denote by $\operatorname{diam}_{d_{g}}(S)$ the diameter of $S$ w.r.t. $d_{g}$. Assuming the behaviors of $w(r)$ around $r=0$ and $\infty$, we have the following estimates. These are very useful to control the $d_{g}$-Cauchy sequences $\left\{\left(r_{k}, y_{k}\right)\right\} \subset$ $\mathbb{R}_{>0} \times Y$ with $\lim _{k \rightarrow \infty} r_{k}=0$ or $\infty$.

Lemma 2.25. For any $\left(r_{0}, y_{0}\right),\left(r_{1}, y_{1}\right) \in \mathbb{R}_{>0} \times Y$, we have the following.

(1) If $T_{0} \in \mathbb{R}$ and $\lim _{r \rightarrow 0} w(r)=0$, we have

$$
d_{g}\left(\left(r_{0}, y_{0}\right),\left(r_{1}, y_{1}\right)\right) \leq T\left(r_{0}\right)+T\left(r_{1}\right)-2 T_{0} .
$$

In particular, we have for $R>0$

$$
\operatorname{diam}_{d_{g}}\left\{(r, y) \in \mathbb{R}_{>0} \times Y \mid r \leq R\right\} \leq 2\left(T(R)-T_{0}\right) .
$$

(2) If $T_{\infty} \in \mathbb{R}$ and $\lim _{r \rightarrow \infty} w(r)=0$, we have

$$
d_{g}\left(\left(r_{0}, y_{0}\right),\left(r_{1}, y_{1}\right)\right) \leq 2 T_{\infty}-T\left(r_{0}\right)-T\left(r_{1}\right) .
$$

In particular, we have for $R>0$

$$
\operatorname{diam}_{d_{g}}\left\{(r, y) \in \mathbb{R}_{>0} \times Y \mid r \geq R\right\} \leq 2\left(T_{\infty}-T(R)\right) .
$$

Remark 2.26. If $T_{0} \in \mathbb{R}$, we easily see $\liminf _{r \rightarrow 0} w(r)=0$. However, $T_{0} \in \mathbb{R}$ does not imply $\lim _{r \rightarrow 0} w(r)=0$.

Indeed, setting $q=e^{x}$ for $x \in \mathbb{R}$ and defining $u: \mathbb{R} \rightarrow \mathbb{R}_{>0}$ by $u(x)=$ $\sqrt{k w\left(e^{x}\right)}$, the condition $T_{0} \in \mathbb{R}$ is equivalent to $\int_{-\infty}^{-1} u(x) d x<\infty$. Suppose that $u(x)=\frac{1}{x^{2}}+S(x)$ for $x \in(-\infty,-1]$, where $S: \mathbb{R} \rightarrow \mathbb{R}$ is given by

$S(x)=\sum_{n \in \mathbb{Z}-\{0\}} S_{n}(x), \quad S_{n}(x)=\left\{\begin{array}{ll}n^{2}\left(x-n+\frac{1}{n^{2}}\right) & \text { for } x \in\left[n-\frac{1}{n^{2}}, n\right] \\ n^{2}\left(-x+n+\frac{1}{n^{2}}\right) & \text { for } x \in\left[n, n+\frac{1}{n^{2}}\right], \\ 0 & \text { otherwise. }\end{array}\right.$, 
Then we see that $\int_{-\infty}^{-1} u(x) d x<\infty$ and $\lim _{x \rightarrow-\infty} u(x) \neq 0$. Though the function $S$ is not smooth, we may replace $S$ with a smooth function which approximates S. Similar statement also holds for $T_{\infty}$.

Proof. For any path $c$ connecting $\left(r_{0}, y_{0}\right)$ and $\left(r_{1}, y_{1}\right)$, we have $d_{g}\left(\left(r_{0}, y_{0}\right),\left(r_{1}, y_{1}\right)\right) \leq$ $L_{g}(c)$. We will take the following path to show (2.25) and (2.26).

Fixing $s>0$, define $c_{1}, c_{2}, c_{3}:[0,1] \rightarrow \mathbb{R}_{>0} \times Y$ by

$$
\begin{aligned}
& c_{1}(t)=\left(((s-1) t+1) r_{0}, y_{0}\right), \\
& c_{2}(t)=\left(s\left(\left(r_{1}-r_{0}\right) t+r_{0}\right), \tilde{y}(t)\right), \\
& c_{3}(t)=\left(((s-1)(1-t)+1) r_{1}, y_{1}\right),
\end{aligned}
$$

where $\tilde{y}:[0,1] \rightarrow Y$ is a path such that $\tilde{y}(0)=y_{0}$ and $\tilde{y}(1)=y_{1}$. That is, $c_{1}$ is a path connecting $\left(r_{0}, y_{0}\right)$ and $\left(s r_{0}, y_{0}\right), c_{2}$ is a path connecting $\left(s r_{0}, y_{0}\right)$ and $\left(s r_{1}, y_{1}\right)$, and $c_{3}$ is a path connecting $\left(s r_{1}, y_{1}\right)$ and $\left(r_{1}, y_{1}\right)$. Define $c:[0,1] \rightarrow$ $\mathbb{R}_{>0} \times Y$ by the concatenation of these paths:

$$
c=c_{1} * c_{2} * c_{3} .
$$

Then we compute

$$
\begin{aligned}
L_{g}\left(c_{1}\right) & =\int_{0}^{1} r_{0}|s-1| \frac{\sqrt{k w\left(r \circ c_{1}\right)}}{r \circ c_{1}} d t \\
& =\left|\int_{0}^{1} r_{0}(s-1) \frac{\sqrt{k w\left(r \circ c_{1}\right)}}{r \circ c_{1}} d t\right|=\left|\int_{0}^{1} \frac{d}{d t} T\left(\left(r \circ c_{1}\right)\right) d t\right|=\left|T\left(s r_{0}\right)-T\left(r_{0}\right)\right| .
\end{aligned}
$$

Similarly, we obtain $L\left(c_{3}\right)=\left|T\left(s r_{1}\right)-T\left(r_{1}\right)\right|$. We also have

$$
L_{g}\left(c_{2}\right) \leq I_{3}+I_{4}
$$

where

$$
\begin{aligned}
& I_{3}=\int_{0}^{1}\left|s\left(r_{1}-r_{0}\right)\right| \frac{\sqrt{k w\left(r \circ c_{2}\right)}}{r \circ c_{2}} d t=\left|\int_{0}^{1} \frac{d}{d t} T\left(\left(r \circ c_{2}\right)\right) d t\right|=\left|T\left(s r_{1}\right)-T\left(s r_{0}\right)\right| \text {, } \\
& I_{4}=\int_{0}^{1} \sqrt{w\left(r \circ c_{2}\right)\left|\frac{d \tilde{y}}{d t}(t)\right|_{g_{Y}}^{2}} d t=\int_{0}^{1} \sqrt{w\left(r \circ c_{2}\right)}\left|\frac{d \tilde{y}}{d t}(t)\right|_{g_{Y}} d t
\end{aligned}
$$

Since $\left(r \circ c_{2}\right)(t) \in\left[\operatorname{smin}\left\{r_{0}, r_{1}\right\}, s \max \left\{r_{0}, r_{1}\right\}\right]$ for any $t \in[0,1]$, setting

$$
C^{\prime \prime \prime}=C^{\prime \prime \prime}\left(s, r_{0}, r_{1}, w\right)=\max \left\{\sqrt{w(r)} \mid r \in\left[\operatorname{smin}\left\{r_{0}, r_{1}\right\}, \operatorname{smax}\left\{r_{0}, r_{1}\right\}\right]\right\},
$$

we see that

$$
I_{4} \leq C^{\prime \prime \prime} L_{g_{Y}}(\tilde{y})
$$

Summarizing these estimates, we obtain

$$
\begin{aligned}
d_{g}\left(\left(r_{0}, y_{0}\right),\left(r_{1}, y_{1}\right)\right) \leq & \left|T\left(s r_{0}\right)-T\left(r_{0}\right)\right|+\left|T\left(s r_{1}\right)-T\left(r_{1}\right)\right| \\
& +\left|T\left(s r_{1}\right)-T\left(s r_{0}\right)\right|+C^{\prime \prime \prime} L_{g_{Y}}(\tilde{y}) .
\end{aligned}
$$

Now suppose that $T_{0} \in \mathbb{R}$ and $\lim _{r \rightarrow 0} w(r)=0$. Then we have $\lim _{s \rightarrow 0} C^{\prime \prime \prime}=$ 0 . Letting $s \rightarrow 0$ in (2.27), we obtain

$$
d_{g}\left(\left(r_{0}, y_{0}\right),\left(r_{1}, y_{1}\right)\right) \leq\left|T_{0}-T\left(r_{0}\right)\right|+\left|T_{0}-T\left(r_{1}\right)\right|=T\left(r_{0}\right)+T\left(r_{1}\right)-2 T_{0} .
$$


Next, suppose that $T_{\infty} \in \mathbb{R}$ and $\lim _{r \rightarrow \infty} w(r)=0$. Then we have $\lim _{s \rightarrow \infty} C^{\prime \prime \prime}=$ 0 . Letting $s \rightarrow \infty$ in (2.27), we obtain

$$
d_{g}\left(\left(r_{0}, y_{0}\right),\left(r_{1}, y_{1}\right)\right) \leq\left|T_{\infty}-T\left(r_{0}\right)\right|+\left|T_{\infty}-T\left(r_{1}\right)\right|=2 T_{\infty}-T\left(r_{0}\right)-T\left(r_{1}\right) .
$$

From these lemmas, we can determine the metric completion of $\mathbb{R}_{>0} \times Y$ w.r.t. $d_{g}$.

Theorem 2.27. The metric completion $\overline{\mathbb{R}_{>0} \times Y}$ of $\mathbb{R}_{>0} \times Y$ w.r.t. the metric $d_{g}$ induced from the Riemannian metric $g=g(w)$ given in (2.15) is homeomorphic to the following.

(1) If $T_{0}=-\infty$ and $T_{\infty}=\infty$,

$$
\mathbb{R}_{>0} \times \bar{Y} \quad \text { with the product topology }
$$

(2) If $T_{0} \in \mathbb{R}, T_{\infty}=\infty$ and $\lim _{r \rightarrow 0} w(r)=0$,

$$
\left(\{0\} \cup \mathbb{R}_{>0}\right) \times \bar{Y} /(\{0\} \times \bar{Y})=\left(\mathbb{R}_{>0} \times \bar{Y}\right) \cup\{*\}
$$

with the topology $\mathcal{O}_{0}$ given below.

(3) If $T_{0}=-\infty, T_{\infty} \in \mathbb{R}$ and $\lim _{r \rightarrow \infty} w(r)=0$,

$$
\left(\mathbb{R}_{>0} \cup\{\infty\}\right) \times \bar{Y} /(\{\infty\} \times \bar{Y})=\left(\mathbb{R}_{>0} \times \bar{Y}\right) \cup\{*\}
$$

with the topology $\mathcal{O}_{\infty}$ given below.

(4) If $T_{0} \in \mathbb{R}, T_{\infty} \in \mathbb{R}, \lim _{r \rightarrow 0} w(r)=0$ and $\lim _{r \rightarrow \infty} w(r)=0$,

$$
\left(\{0\} \cup \mathbb{R}_{>0} \cup\{\infty\}\right) \times \bar{Y} /(\{0, \infty\} \times \bar{Y})=\left(\mathbb{R}_{>0} \times \bar{Y}\right) \cup\{*\} \cup\{*\}
$$

with the topology $\mathcal{O}_{0, \infty}$ given below.

Here, $\bar{Y}$ is the metric completion of $Y$ w.r.t. the metric $d_{g_{Y}}$ induced from $g_{Y}$. Let $\pi_{0}:\left(\{0\} \cup \mathbb{R}_{>0}\right) \times \bar{Y} \rightarrow\left(\{0\} \cup \mathbb{R}_{>0}\right) \times \bar{Y} /(\{0\} \times \bar{Y})$ be the projection. Set $*_{0}=\pi_{0}(\{0\} \times \bar{Y})$. The topology $\mathcal{O}_{0}$ is defined by the fundamental system of neighborhoods $\mathcal{U}(x)$ given below. If $x \neq *_{0}, \mathcal{U}(x)$ consists of $\epsilon$-balls centered at $x$ for $\epsilon>0$ w.r.t. the product metric. If $x=*_{0}$, we set

$$
\mathcal{U}\left(*_{0}\right)=\left\{\pi_{0}([0, \epsilon) \times \bar{Y}) \mid \epsilon>0\right\} .
$$

Let $\pi_{\infty}:\left(\mathbb{R}_{>0} \cup\{\infty\}\right) \times \bar{Y} \rightarrow\left(\mathbb{R}_{>0} \cup\{\infty\}\right) \times \bar{Y} /(\{\infty\} \times \bar{Y})$ be the projection. Set $*_{\infty}=\pi_{\infty}(\{\infty\} \times \bar{Y})$. The topology $\mathcal{O}_{\infty}$ is given by the fundamental system of neighborhoods $\mathcal{U}(x)$ given below. If $x \neq *_{\infty}, \mathcal{U}(x)$ consists of $\epsilon$-balls centered at $x$ for $\epsilon>0$ w.r.t. the product metric. If $x=*_{\infty}$, we $\operatorname{set} \mathcal{U}\left(*_{\infty}\right)=\left\{\pi_{\infty}((R, \infty] \times\right.$ $\bar{Y}) \mid R>0\}$. The topology $\mathcal{O}_{0, \infty}$ is similarly defined by setting the fundamental systems of neighborhoods as above.

Remark 2.28. Roughly speaking, the metric completion is the cylinder of $\bar{Y}$ in the case (1), the cone (with the apex) of $\bar{Y}$ in the cases (2) and (3), and the suspension of $\bar{Y}$ in the case (4).

In general, the topologies $\mathcal{O}_{0}, \mathcal{O}_{\infty}$ and $\mathcal{O}_{0, \infty}$ are weaker than the quotient topologies. If $\bar{Y}$ is compact, they agree with the quotient topologies. In particular, in the case (4), the metric completion $\overline{\mathbb{R}_{>0} \times Y}$ is compact if $\bar{Y}$ is compact because there is a surjection from $\left(\{0\} \cup \mathbb{R}_{>0} \cup\{\infty\}\right) \times \bar{Y} \cong[0,1] \times \bar{Y}$. 
Proof. Use the notation of Definition A.2. Consider the case (1). Define a map

$$
\Theta_{1}: \overline{\mathbb{R}_{>0} \times Y} \rightarrow \mathbb{R}_{>0} \times \bar{Y}, \quad\left[\left(r_{k}, y_{k}\right)\right] \mapsto\left(\lim _{k \rightarrow \infty} r_{k},\left[y_{k}\right]\right)
$$

This map is well-defined. Indeed, by Corollary 2.23 , we have $\lim _{k \rightarrow \infty} r_{k} \in \mathbb{R}_{>0}$. Then we may assume that $\left\{\left(r_{k}, y_{k}\right)\right\} \subset\left(R_{1}, R_{2}\right) \times Y$ for some $0<R_{1}<$ $R_{2}$. Then Lemma 2.24 (1) implies that $\left\{y_{k}\right\}$ is a $d_{g_{Y}}$-Cauchy sequence. If $\lim _{k \rightarrow \infty} d_{g}\left(\left(r_{k}, y_{k}\right),\left(r_{k}^{\prime}, y_{k}^{\prime}\right)\right)=0$ for $d_{g}$-Cauchy sequences $\left\{\left(r_{k}, y_{k}\right)\right\}$ and $\left\{\left(r_{k}^{\prime}, y_{k}^{\prime}\right)\right\}$, Lemma 2.22 and Lemma 2.24 (1) imply that $\lim _{k \rightarrow \infty} r_{k}=\lim _{k \rightarrow \infty} r_{k}^{\prime}$ and $\lim _{k \rightarrow \infty} d_{g}\left(y_{k}, y_{k}^{\prime}\right)=0$, and hence $\Theta_{1}$ is well-defined.

We show that $\Theta_{1}$ is bijective. For any $\left(r_{0},\left[y_{k}\right]\right) \in \mathbb{R}_{>0} \times \bar{Y},\left\{\left(r_{0}, y_{k}\right)\right\}$ is a $d_{g}$-Cauchy sequence by Lemma 2.24 (2). Hence we see that $\Theta_{1}$ is surjective. Suppose that $\lim _{k \rightarrow \infty} r_{k}=\lim _{k \rightarrow \infty} r_{k}^{\prime}$ and $\lim _{k \rightarrow \infty} d_{g}\left(y_{k}, y_{k}^{\prime}\right)=0$ for $d_{g}$-Cauchy sequences $\left\{\left(r_{k}, y_{k}\right)\right\}$ and $\left\{\left(r_{k}^{\prime}, y_{k}^{\prime}\right)\right\}$. Then Lemma 2.24 (2) implies that $\lim _{k \rightarrow \infty} d_{g}\left(\left(r_{k}, y_{k}\right),\left(r_{k}^{\prime}, y_{k}^{\prime}\right)\right)=0$, and hence $\Theta_{1}$ is injective.

We show that $\Theta_{1}$ is homeomorphic. Let $\left\{\left[\left(r_{k j}, y_{k j}\right)\right]\right\}_{j}$ be a sequence in $\overline{\mathbb{R}_{>0} \times Y}$ converging to $\left[\left(r_{k}, y_{k}\right)\right]$. That is,

$$
\lim _{j \rightarrow \infty} \lim _{k \rightarrow \infty} d_{g}\left(\left(r_{k j}, y_{k j}\right),\left(r_{k}, y_{k}\right)\right)=0 .
$$

By Lemma 2.22, we have $\lim _{j \rightarrow \infty} \lim _{k \rightarrow \infty}\left|r_{k j}-r_{k}\right|=0$. Since $\lim _{k \rightarrow \infty} r_{k}>0$, we can apply Lemma 2.24 (1) and it follows that $\lim _{j \rightarrow \infty} \lim _{k \rightarrow \infty} d_{g_{Y}}\left(y_{k j}, y_{k}\right)=$ 0 . Hence $\Theta_{1}$ is continuous.

Let $\left\{\left(r_{0 j},\left[y_{k j}\right]\right)\right\}_{j}$ be a sequence in $\mathbb{R}_{>0} \times \bar{Y}$ converging to $\left(r_{0},\left[y_{k}\right]\right)$. Since $\Theta_{1}^{-1}\left(r_{0},\left[y_{k}\right]\right)=\left[\left(r_{0}, y_{k}\right)\right]$, Lemma $2.24(2)$ implies that

$$
\lim _{j \rightarrow \infty} d_{g}\left(\left[\left(r_{0 j}, y_{k j}\right)\right],\left[\left(r_{0}, y_{k}\right)\right]\right)=\lim _{j \rightarrow \infty} \lim _{k \rightarrow \infty} d_{g}\left(\left(r_{0 j}, y_{k j}\right),\left(r_{0}, y_{k}\right)\right)=0
$$

Hence $\Theta_{1}^{-1}$ is continuous.

Next, we consider the case (2). Define a map $\Theta_{2}: \overline{\mathbb{R}_{>0} \times Y} \rightarrow\left(\{0\} \cup \mathbb{R}_{>0}\right) \times$ $\bar{Y} /(\{0\} \times \bar{Y})$ by

$$
\Theta_{2}\left(\left[\left(r_{k}, y_{k}\right)\right]\right)= \begin{cases}\left(\lim _{k \rightarrow \infty} r_{k},\left[y_{k}\right]\right) & \text { if } \quad \lim _{k \rightarrow \infty} r_{k}>0 \\ *_{0} & \text { if } \quad \lim _{k \rightarrow \infty} r_{k}=0\end{cases}
$$

This map is well-defined and bijective. Indeed, Corollary 2.23 implies that $\lim _{k \rightarrow \infty} r_{k} \in\{0\} \cup \mathbb{R}_{>0}$. Every $d_{g}$-Cauchy sequence with $\lim _{k \rightarrow \infty} r_{k}>0$ corresponds to an element of $\mathbb{R}_{>0} \times \bar{Y}$ as in the case (1). For $d_{g}$-Cauchy sequences $\left\{\left(r_{k}, y_{k}\right)\right\}$ and $\left\{\left(r_{k}^{\prime}, y_{k}^{\prime}\right)\right\}$ such that $\lim _{k \rightarrow \infty} r_{k}=\lim _{k \rightarrow \infty} r_{k}^{\prime}=0$, Lemma 2.25 (1) implies that $\lim _{k \rightarrow \infty} d_{g}\left(\left(r_{k}, y_{k}\right),\left(r_{k}^{\prime}, y_{k}^{\prime}\right)\right)=0$. Hence $\Theta_{2}$ is well-defined and bijective.

We show that $\Theta_{2}$ is homeomorphic. Denote by $*$ the unique equivalence class $\left[\left(r_{k}, y_{k}\right)\right] \in \overline{\mathbb{R}_{>0} \times Y}$ such that $\lim _{k \rightarrow \infty} r_{k}=0$. By (1), we see that

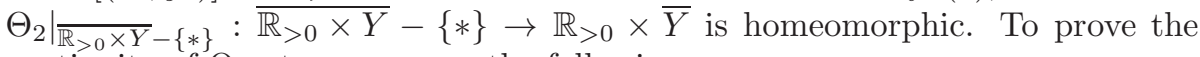
continuity of $\Theta_{2}$ at $*$, we prove the following.

Lemma 2.29. The fundamental system of neighborhoods at * w.r.t. the topology induced from $d_{g}$ is given by

$$
\left\{U_{\epsilon} \mid \epsilon>0\right\} \quad \text { where } \quad U_{\epsilon}=\left\{\left[\left(r_{k}, y_{k}\right)\right] \in \overline{\mathbb{R}_{>0} \times Y} \mid \lim _{k \rightarrow \infty} r_{k}<\epsilon\right\} .
$$


Proof. Since $\left(\overline{\mathbb{R}_{>0} \times Y}, d_{g}\right)$ is a metric space, the fundamental system of neighborhoods at $*$ consists of the $\delta$-balls $B_{\delta}$ centered at $*$ for $\delta>0$. Hence we only have to show that for any $\delta>0$, there exists $\epsilon>0$ such that $U_{\epsilon} \subset B_{\delta}$.

Since the function $T$ in (2.23) is continuous at 0 under the assumption of (2), for any $\delta>0$, there exists $\epsilon>0$ such that $r<\epsilon \Rightarrow T(r)-T_{0}<\delta$. Then (2.25) implies that for any $\left[\left(r_{k}, y_{k}\right)\right] \in U_{\epsilon}$,

$$
d_{g}\left(*,\left[\left(r_{k}, y_{k}\right)\right]\right) \leq \lim _{k \rightarrow \infty} T\left(r_{k}\right)-T_{0}<\delta,
$$

which implies that $U_{\epsilon} \subset B_{\delta}$.

Then since $\Theta_{2}\left(U_{\epsilon}\right)=\pi_{0}([0, \epsilon) \times \bar{Y})$, we see that $\Theta_{2}$ is continuous at $*$ and $\Theta_{2}^{-1}$ is continuous at $*_{0}$. We can prove (3) and (4) similarly.

Finally, we give a description of $\bar{Y}$ in terms of $\overline{\mathbb{R}_{>0} \times Y}$. The following implies that we can recover $\bar{Y}$ from $\overline{\mathbb{R}_{>0} \times Y}$.

Proposition 2.30. Use the notation of Definition A.2. For any $R>0$, the map

$$
I_{R}: \bar{Y} \rightarrow\left\{\left[\left(r_{k}, y_{k}\right)\right] \in \overline{\mathbb{R}_{>0} \times Y} \mid \lim _{k \rightarrow \infty} r_{k}=R\right\}, \quad\left[y_{k}\right] \mapsto\left[\left(R, y_{k}\right)\right]
$$

is homeomorphic.

Proof. The proof is similar to that of Theorem 2.27. Let $\left\{y_{k}\right\}$ is a $d_{g_{Y}}$-Cauchy sequence. Then $\left\{\left(R, y_{k}\right)\right\}$ is a $d_{g}$-Cauchy sequence by Lemma $2.24(2)$. Hence $I_{R}$ is well-defined.

Let $\left\{\left(r_{k}, y_{k}\right)\right\}$ be a $d_{g}$-Cauchy sequence with $\lim _{k \rightarrow \infty} r_{k}=R$. Then Lemma 2.24 (1) implies that $\left\{y_{k}\right\}$ is a $d_{g_{Y}}$-Cauchy sequence. By Lemma 2.24 (2), we have

$$
d_{g}\left(\left(r_{k}, y_{k}\right),\left(R, y_{k}\right)\right) \leq\left|T\left(r_{k}\right)-T(R)\right| \rightarrow 0 \quad \text { as } \quad k \rightarrow \infty .
$$

Then $\left\{\left(r_{k}, y_{k}\right)\right\} \sim\left\{\left(R, y_{k}\right)\right\}$, and hence $I_{R}$ is surjective.

Suppose that $\lim _{k \rightarrow \infty} d_{g}\left(\left(R, y_{k}\right),\left(R, y_{k}^{\prime}\right)\right)=0$ for $d_{g_{Y}}$-Cauchy sequences $\left\{y_{k}\right\}$ and $\left\{y_{k}^{\prime}\right\}$. Then Lemma 2.24(1) implies that $\lim _{k \rightarrow \infty} d_{g_{Y}}\left(y_{k}, y_{k}^{\prime}\right)=0$, and hence $I_{R}$ is injective.

We show that $I_{R}$ is homeomorphic. Let $\left\{\left[y_{k j}\right]\right\}_{j}$ be a sequence in $\bar{Y}$ converging to $\left[y_{k}\right]$. Then by Lemma $2.24(2), \lim _{j \rightarrow \infty} d_{g}\left(\left[\left(R, y_{k j}\right],\left[\left(R, y_{k}\right)\right]\right)=0\right.$, and hence $I_{R}$ is continuous. By the proof above, we have $I_{R}^{-1}\left(\left[\left(r_{k}, y_{k}\right)\right]\right)=\left[y_{k}\right]$. Then by Lemma 2.24 (1), we see that $I_{R}^{-1}$ is continuous.

Remark 2.31. Thus if we know $\overline{\mathbb{R}_{>0} \times Y}$, we see $\bar{Y}$. In particular, by Theorem 2.27, if we know $\overline{\left(\mathbb{R}_{>0} \times Y, d_{g(w)}\right)}$, the metric completion of $\mathbb{R}_{>0} \times Y$ w.r.t. $d_{g(w)}$, for one $w$, we can obtain $\overline{\left(\mathbb{R}_{>0} \times Y, d_{g(\tilde{w})}\right)}$ for $\tilde{w}$ satisfying one of four assumptions in Theorem 2.27.

\section{Conformal transformations of the pseudo-Riemannian metric of a homogeneous pair}

\subsection{The splitting theorem}

In this section, we give the definition of a homogeneous pair for a pseudoRiemannian metric $g$ and a positive function $f$ on a manifold $M$ admitting a 
free $\mathbb{R}_{>0}$-action in more detail. Then we study the geometric structures of the pseudo-Riemannian manifold $(M,(v \circ f) g)$, where $v: \mathbb{R}_{>0} \rightarrow \mathbb{R}_{>0}$ is a smooth function.

Definition 3.1 (Definition 1.1). Let $(M, g)$ be a pseudo-Riemannian manifold which admits a free $\mathbb{R}_{>0}$-action. Denote by $m: \mathbb{R}_{>0} \times M \rightarrow M$ the $\mathbb{R}_{>0}$-action and set $m_{\lambda}=m(\lambda, \cdot)$ for $\lambda \in \mathbb{R}_{>0}$. Let $P \in \mathfrak{X}(M)$ be a vector field generated by the $\mathbb{R}_{>0}$-action. That is,

$$
P_{x}=\left.\frac{d}{d t} m\left(e^{t}, x\right)\right|_{t=0}
$$

for $x \in M$. Suppose that $f: M \rightarrow \mathbb{R}_{>0}$ is a smooth function and $\alpha \in \mathbb{R}-\{0\}$.

The pair $(g, f)$ is called $a$ homogeneous pair of degree $\alpha$ if

$$
\begin{aligned}
m_{\lambda}^{*} g & =\lambda^{\alpha} g, \\
m_{\lambda}^{*} f & =\lambda^{\alpha} f, \\
g(P, \cdot) & =d f
\end{aligned}
$$

for any $\lambda>0$.

Remark 3.2. The degree of $g$ must be equal to that of $f$. That is, if $m_{\lambda}^{*} g=\lambda^{\alpha} g$ and $m_{\lambda}^{*} f=\lambda^{\beta} f$, the equation $g(P, \cdot)=d f$ implies that $\alpha=\beta$.

Indeed, by $g(P, \cdot)=d f$, we have

$$
m_{\lambda}^{*}(g(P, \cdot))=m_{\lambda}^{*} d f=\lambda^{\beta} d f .
$$

Since $P_{m_{\lambda}(x)}=\left.\frac{d}{d t} m_{e^{t}} m_{\lambda}(x)\right|_{t=0}=\left(m_{\lambda}\right)_{*} P_{x}$ for any $x \in M$, we compute

$$
m_{\lambda}^{*}(g(P, \cdot))=\left(g \circ m_{\lambda}\right)\left(P \circ m_{\lambda},\left(m_{\lambda}\right)_{*}(\cdot)\right)=\left(m_{\lambda}^{*} g\right)(P, \cdot)=\lambda^{\alpha} g(P, \cdot)=\lambda^{\alpha} d f .
$$

Hence we obtain $\alpha=\beta$.

We first show that $(M,(v \circ f) g)$ admits the structure of a warped product. This is a generalization of the splitting theorem for Hessian manifolds that are cones given in Loftin, Theorem 1] and [Totaro, Lemmas 2.1 and 2.4] (cf. Remark 4.4).

Theorem 3.3. Let $(M, g)$ be a pseudo-Riemannian manifold which admits a free $\mathbb{R}_{>0}$-action and let $f: M \rightarrow \mathbb{R}_{>0}$ be a smooth function. Suppose that $(g, f)$ is a homogeneous pair of degree $\alpha$. Then

(1) we have $(d f)_{x} \neq 0$ for any $x \in M$. Thus for any $l>0$

$$
M_{l}=\{x \in M \mid f(x)=l\}
$$

is a submanifold of $M$. Denote by $g_{l}$ the pullback of $g$ to $M_{l}$. Then $g_{l}$ is a pseudo-Riemannian metric on $M_{l}$.

(2) For a function $v: \mathbb{R}_{>0} \rightarrow \mathbb{R}_{>0}$, the map

$$
\psi: \mathbb{R}_{>0} \times M_{l} \rightarrow M, \quad(r, y) \mapsto m\left(\left(\frac{r}{l}\right)^{\frac{1}{\alpha}}, y\right)
$$

gives an isometry between $\left(\mathbb{R}_{>0} \times M_{l}, v(r)\left(\frac{1}{\alpha r} d r^{2}+\frac{r}{l} g_{l}\right)\right)$ and $(M,(v \circ$ f)g). 
Remark 3.4. For $l_{1}, l_{2}>0$, the diffeomorphism

$$
\psi_{l_{1}, l_{2}}: M_{l_{1}} \rightarrow M_{l_{2}}, \quad x \mapsto m\left(\left(\frac{l_{2}}{l_{1}}\right)^{1 / \alpha}, x\right)
$$

gives an isometry $\left(M_{l_{1}}, g_{l_{1}} / l_{1}\right) \cong\left(M_{l_{2}}, g_{l_{2}} / l_{2}\right)$ by (3.1). Hence the isometry (3.4) is independent of $l>0$.

Remark 3.5. We do not use the local coordinates to prove Theorem 3.3. Thus the statement formally holds when $M$ is infinite dimensional.

The subtle point in the infinite dimensional case is the notion of submanifolds. In (1), we use implicit function theorem to prove that $M_{l}$ is a submanifold of $M$ by $(d f)_{x} \neq 0$. However, there is no implicit function theorem in the infinite dimensional case in general. (For example, if $M$ is a Banach manifold, there is an implicit function theorem.) For the details of the theory of infinite dimensional manifolds, see [KM, Lang].

Remark 3.6. By Theorem 3.3, we have an isometry between $(M,(v \circ f) g)$ and $\left(\mathbb{R}_{>0} \times M_{l}, v(r)\left(\frac{1}{\alpha r} d r^{2}+\frac{r}{l} g_{l}\right)\right)$. Setting

$$
k=\frac{l}{\alpha}, \quad w(r)=\frac{r v(r)}{l} \quad \text { and } \quad\left(Y, g_{Y}\right)=\left(M_{l}, g_{l}\right)
$$

this pseudo-Riemannian metric is of the form $g(w)$ in 2.15).

Proof of Theorem 3.3. First, we prove (1). For any $x \in M$, we compute

$$
(d f)_{x}\left(P_{x}\right)=\left.(d f)_{x}\left(\left.\frac{d}{d t} m\left(e^{t}, x\right)\right|_{t=0}\right) \stackrel{3.2}{=} \frac{d}{d t} e^{\alpha t} f(x)\right|_{t=0}=\alpha f(x) .
$$

Since $f$ is a positive function, we see that $(d f)_{x} \neq 0$. For $y \in M_{l}$, we have the decomposition

$T_{y} M=T_{y} M_{l} \oplus \mathbb{R} P_{y}=\operatorname{ker}(d f)_{y} \oplus \mathbb{R} P_{y}, \quad A=\left(A-\frac{(d f)_{y}(A)}{\alpha l} P_{y}\right)+\frac{(d f)_{y}(A)}{\alpha l} P_{y}$,

which is orthogonal by (3.3). Then it is immediate to see that $g_{l}$ is a pseudoRiemannian metric on $M_{l}$.

Next, we prove (2). Since the inverse $\psi^{-1}: M \rightarrow \mathbb{R}_{>0} \times M_{l}$ is given by

$$
\psi^{-1}(x)=\left(f(x), m\left(\left(\frac{l}{f(x)}\right)^{\frac{1}{\alpha}}, x\right)\right),
$$

we see that $\psi$ is a diffeomorphism. We compute $\psi^{*}((v \circ f) g)$ to show that $\psi$ is an isometry. For $(r, y) \in \mathbb{R}_{>0} \times M_{l}$, we have

$$
\psi^{*}(v \circ f)(r, y)=v\left(f\left(m\left(\left(\frac{r}{l}\right)^{\frac{1}{\alpha}}, y\right)\right)\right) \stackrel{\sqrt[3.2]{=}}{=} v(r) .
$$


Thus we only have to compute $\left(\psi^{*} g\right)_{(r, y)}\left(\partial_{r}, \partial_{r}\right),\left(\psi^{*} g\right)_{(r, y)}\left(\partial_{r}, a\right)$, and $\left(\psi^{*} g\right)_{(r, y)}(a, a)$ for any $a \in \operatorname{ker}(d f)_{y}$. Since

$$
\begin{aligned}
\left(\psi_{*}\right)_{(r, y)}\left(\partial_{r}\right) & =\left.\frac{d}{d s} m\left(\left(\frac{r+s}{l}\right)^{\frac{1}{\alpha}}, y\right)\right|_{s=0} \\
& =\left.\frac{d}{d s} m_{\left(\frac{r}{l}\right)^{\frac{1}{\alpha}}} \circ m_{\left(1+\frac{s}{r}\right)^{\frac{1}{\alpha}}}(y)\right|_{s=0}=\frac{1}{\alpha r}\left(m_{\left(\frac{r}{l}\right)^{\frac{1}{\alpha}}}\right)_{*} P_{y},
\end{aligned}
$$

we have by (3.1), 3.3) and (3.5)

$$
\left(\psi^{*} g\right)_{(r, y)}\left(\partial_{r}, \partial_{r}\right)=\frac{1}{(\alpha r)^{2}} \frac{r}{l} g\left(P_{y}, P_{y}\right)=\frac{1}{(\alpha r)^{2}} \cdot \frac{r}{l} \cdot \alpha l=\frac{1}{\alpha r} .
$$

Since

$$
\left(\psi_{*}\right)_{(r, y)} a=\left(m_{\left(\frac{r}{l}\right)^{\frac{1}{\alpha}}}\right)_{*} a,
$$

we have $\left(\psi^{*} g\right)_{(r, y)}\left(\partial_{r}, a\right)=0$ by (3.1) and (3.3). By (3.1), we obtain

$$
\left(\psi^{*} g\right)_{(r, y)}(a, a)=\frac{r}{l} \cdot g(a, a) .
$$

Hence the proof is completed.

Note that there is the following isometry between $(M,(v \circ f) g)$ and $(M,(\tilde{v} \circ$ $f) g$ ) for some $\tilde{v}: \mathbb{R}_{>0} \rightarrow \mathbb{R}_{>0}$. Hence they have the same sectional curvature, geodesics and the metric completion.

Lemma 3.7. In the setting of Theorem 3.3, the pseudo-Riemannian manifolds $(M,(v \circ f) g)$ and $\left(M, \frac{1}{f^{2}} v\left(\frac{1}{f}\right) g\right)$ are isometric via $\psi_{M}: M \rightarrow M$ defined by $\psi_{M}(x)=m\left(f(x)^{-2 / \alpha}, x\right)$.

Proof. Recall Remark 3.6 By Remark 2.15, we have an isometry

$$
\begin{aligned}
\left(\mathbb{R}_{>0} \times M_{l}, v(r)\left(\frac{1}{\alpha r} d r^{2}+\frac{r}{l} g_{l}\right)\right) & \cong\left(\mathbb{R}_{>0} \times M_{l}, v\left(\frac{1}{r}\right)\left(\frac{r}{\alpha}\left(d\left(\frac{1}{r}\right)\right)^{2}+\frac{1}{l r} g_{l}\right)\right) \\
& =\left(\mathbb{R}_{>0} \times M_{l}, \frac{1}{r^{2}} v\left(\frac{1}{r}\right)\left(\frac{1}{\alpha r} d r^{2}+\frac{r}{l} g_{l}\right)\right)
\end{aligned}
$$

via $j:(r, y) \mapsto(1 / r, y)$. Since the map $\psi$ in (3.4) gives an isometry between $\left(\mathbb{R}_{>0} \times M_{l}, \frac{1}{r^{2}} v\left(\frac{1}{r}\right)\left(\frac{1}{\alpha r} d r^{2}+\frac{r}{l} g_{l}\right)\right)$ and $\left(M, \frac{1}{f^{2}} v\left(\frac{1}{f}\right) g\right)$, the map $\psi_{M}=\psi \circ j \circ$ $\psi^{-1}: M \rightarrow M$ gives an isometry between $(M,(v \circ f) g)$ and $\left(M, \frac{1}{f^{2}} v\left(\frac{1}{f}\right) g\right)$.

Definition 3.8. Given a homogeneous pair $(g, f)$ of degree $\alpha \in \mathbb{R}-\{0,1\}$, define a new pseudo-Riemannian metric $\hat{g}$ by

$$
\hat{g}=\frac{d f \otimes d f}{f}+(1-\alpha) g .
$$

As we see below, $(\hat{g}, f)$ is also a homogeneous pair of degree $\alpha$. This pseudoRiemannian metric appears in many examples. See Sections 4 and 5 The signature of $\hat{g}$ is different from that of $g$, and hence we can produce a definite pseudo-Riemannian metric in the sense of Definition A.1 in some cases. 
Lemma 3.9. The tensor $\hat{g}$ is a pseudo-Riemannian metric. The pair $(\hat{g}, f)$ is also a homogeneous pair of degree $\alpha$.

Proof. Recalling the decomposition (3.6), suppose that $\hat{g}(k P+a, \cdot)=0$ for $k \in \mathbb{R}$ and $a \in \operatorname{ker}(d f)$. Then we have

$0=\hat{g}(k P+a, P)=\frac{k(d f(P))^{2}}{f}+(1-\alpha) g(k P+a, P) \stackrel{3.3), 3.5}{=} k \alpha^{2} f+k(1-\alpha) \alpha f=k \alpha f$,

and hence we have $k=0$. Then it follows that $\hat{g}(a, \cdot)=(1-\alpha) g(a, \cdot)=0$, which implies that $a=0$. Hence $\hat{g}$ is a pseudo-Riemannian metric.

It is clear to see that $m_{\lambda}^{*} \hat{g}=\lambda^{\alpha} \hat{g}$. By (3.3) for $(g, f)$ and (3.5), we see that $\hat{g}(P, \cdot)=d f$.

By the definition of $\hat{g}$, we see that $\hat{g}_{l}=(1-\alpha) g_{l}$. Then by Theorem 3.3, we have an isometry

$$
(M, \hat{g}) \cong\left(\mathbb{R}_{>0} \times M_{l}, \frac{1}{\alpha r} d r^{2}+(1-\alpha) \frac{r}{l} g_{l}\right) .
$$

Comparing this decomposition with $(M, g) \cong\left(\mathbb{R}_{>0} \times M_{l}, \frac{1}{\alpha r} d r^{2}+\frac{r}{l} g_{l}\right)$, the definiteness of $\hat{g}$ is characterized in terms of the signature of $g$ as follows.

Lemma 3.10. Setting $n=\operatorname{dim} M$, we have the following.

(1) When $\alpha>1, g$ has signature $(1, n-1)$ if and only if $\hat{g}$ is positive definite.

(2) When $0<\alpha<1, g$ is positive definite if and only if $\hat{g}$ is positive definite.

(3) When $\alpha<0, g$ is negative if and only if $\hat{g}$ is negative definite.

\subsection{The sectional curvature}

Let $(g, f)$ be a homogeneous pair on a manifold $M$. By Remark 3.6, we can apply results in Section 2, First, by Proposition 2.13 (3), we can find a function $v: \mathbb{R}_{>0} \rightarrow \mathbb{R}_{>0}$ such that $(v \circ f) g$ has the constant sectional curvature if the level set $\left(M_{l}, g_{l}\right)$ has constant sectional curvature.

Proposition 3.11. Use the notation of Definition 2.12 and Theorem 3.3, Let $(g, f)$ be a homogeneous pair of degree $\alpha$. Suppose that $g_{l}$ has constant sectional curvature $\hat{C}_{l} \in \mathbb{R}: K^{g_{l}}=\hat{C}_{l}$.

Then given $C \in \mathbb{R},(v \circ f) g$ has constant sectional curvature $C$ if

$$
v(r)=\frac{1}{r} w\left(\frac{C}{\alpha}, \frac{l \hat{C}_{l}}{\alpha}, C_{2}, r\right)
$$

where $C_{2} \in \mathbb{R}$ such that $\left(\frac{C}{\alpha}, \frac{l \hat{C}_{l}}{\alpha}, C_{2}\right) \in \Delta_{1} \cup \Delta_{2} \cup \Delta_{3}$.

Proof. By Remark 3.6 and Proposition $2.13(3),(v \circ f) g$ has constant sectional curvature $C$ if

$$
v(r)=\frac{l}{r} w\left(\frac{l C}{\alpha}, C_{1}, C_{2}, r\right)=\frac{1}{r} w\left(\frac{C}{\alpha}, C_{1}, C_{2}, r\right) \quad \text { and } \quad \frac{C_{1} \alpha}{l}=\hat{C}_{l},
$$

where $C_{2} \in \mathbb{R}$ such that $\left(\frac{l C}{\alpha}, C_{1}, C_{2}\right) \in \Delta_{1} \cup \Delta_{2} \cup \Delta_{3}$. The last equation of $v(r)$ follows by Definition 2.12 
Remark 3.12. Remark 3.4 implies that $l K^{g_{l}}$ is independent of $l>0$ because $K^{g_{l} / l}=l K^{g_{l}}$. Thus if $K^{g_{l}}=\hat{C}_{l}, l \hat{C}_{l}$ is independent of $l>0$.

The function $v(r)$ given in Proposition 3.11 is defined for all $r>0$ when $\alpha C \geq 0$. When $\alpha C<0$, it is only defined on the complement of the discrete set of $\mathbb{R}_{>0}$.

To apply Proposition 3.11, $\left(M_{l}, g_{l}\right)$ needs to have constant sectional curvature. This is the case if $g$ is flat. The following is a generalization of Totaro, Corollaries 2.2 and 2.3].

Lemma 3.13. Use the notation of Theorem 3.3. Let $(g, f)$ be a homogeneous pair of degree $\alpha$.

(1) We have

$$
K^{g}(a, b)=\frac{l}{r}\left(K^{g_{l}}(a, b)-\frac{\alpha}{4 l}\right)
$$

for linearly independent $a, b \in T M_{l}$.

(2) The pseudo-Riemannian metric $g$ is flat if and only if $g_{l}$ has constant sectional curvature $\frac{\alpha}{4 l}$.

Proof. Suppose that $v=1$ in Remark 3.6. Since $w(r)=r / l=w(0,1 / 4,-\log l, r)$ in the notation of Definition 2.12 the statement follows from Proposition 2.13 (2) and (3).

The following is immediate from Definition 2.12 Proposition 3.11 and Lemma 3.13. The flatness of $g / f^{2}$ is also implied by Lemma 3.7 .

Corollary 3.14. Let $(g, f)$ be a homogeneous pair of degree $\alpha$. Suppose that $g$ is flat. Then the following holds.

- For $C \in \mathbb{R}$ such that $\alpha C>0$, set

$$
v(r)=\frac{\alpha}{4 C r\left(\cosh \left(\frac{1}{2}\left(\log r+C_{2}\right)\right)\right)^{2}} \quad\left(C_{2} \in \mathbb{R}\right) .
$$

Then $(v \circ f) g$ has constant sectional curvature $C$.

- The pseudo-Riemannian metric $g / f^{2}$ is flat on $M$.

If $g$ is definite in the sense of Definition A.1 and the bound of the sectional curvature of $g_{l}$ is given, we can give the bounds of the sectional curvature of $g$.

Corollary 3.15. Use the notation of Definitions 2.12 and A.1. Let $(g, f)$ be a homogeneous pair of degree $\alpha$. Suppose that $g$ is definite. Given $C \in \mathbb{R}$, set

$$
v(r)=\frac{1}{r} w\left(\frac{C}{\alpha}, C_{1}, C_{2}, r\right),
$$

where $C_{1}, C_{2} \in \mathbb{R}$ such that $\left(\frac{C}{\alpha}, C_{1}, C_{2}\right) \in \Delta_{1} \cup \Delta_{2} \cup \Delta_{3}$. Then

- $K^{(v \circ f) g} \geq C$ when $l K^{g_{l}} \geq \alpha C_{1}$, and

- $K^{(v \circ f) g} \leq C$ when $l K^{g_{l}} \leq \alpha C_{1}$. 
Furthermore, $K^{(v \circ f) g}=C$ if $l K^{g_{l}}=\alpha C_{1}$.

Proof. Suppose that $v(r)=\frac{1}{r} w\left(\frac{C}{\alpha}, C_{1}, C_{2}, r\right)$ in Remark 3.6. Then we have

$$
w(r)=\frac{r v(r)}{l}=\frac{1}{l} w\left(\frac{C}{\alpha}, C_{1}, C_{2}, r\right)=w\left(\frac{l C}{\alpha}, C_{1}, C_{2}, r\right),
$$

and hence the statement follows by Corollary 2.16,

Remark 3.16. In particular, we can apply this when g is flat. By Lemma 3.13, this is the case $K^{g_{l}}=\frac{\alpha}{4 l}$.

More generally, if $K^{g} \geq 0$ (resp. $\left.\leq 0\right)$, we have $l K^{g_{l}} \geq \frac{\alpha}{4}$ (resp. $\left.\leq \frac{\alpha}{4}\right)$. Then by Corollary 3.15, we have $K^{(v \circ f) g} \geq C($ resp. $\leq C)$ for $v(r)=\frac{1}{r} w\left(\frac{C}{\alpha}, \frac{1}{4}, C_{2}, r\right)$, where $C_{2} \in \mathbb{R}$ such that $\left(\frac{C}{\alpha}, \frac{1}{4}, C_{2}\right) \in \Delta_{1} \cup \Delta_{2} \cup \Delta_{3}$.

When $\operatorname{dim} M=2$, we do not need the assumption on $K^{g_{l}}$ by Corollary 2.17 We can prove the following in the same way as Proposition 3.11.

Corollary 3.17. Use the notation of Definition 2.12. Let $(g, f)$ be a homogeneous pair of degree $\alpha$. Suppose that $\operatorname{dim} M=2$.

Then given $C \in \mathbb{R},(v \circ f) g$ has constant sectional curvature $C$ if

$$
v(r)=\frac{1}{r} w\left(\frac{C}{\alpha}, C_{1}, C_{2}, r\right),
$$

where $C_{1}, C_{2} \in \mathbb{R}$ such that $\left(\frac{C}{\alpha}, C_{1}, C_{2}\right) \in \Delta_{1} \cup \Delta_{2} \cup \Delta_{3}$. In particular, setting $C=0$, we see that $f^{\beta} g$ is flat for any $\beta \in \mathbb{R}$.

Corollary 3.17 implies the following, which is a generalization of Totaro, Section 6] for Hessian manifolds.

Remark 3.18. Suppose that $M=M_{1} \times \cdots \times M_{k}$, where $\operatorname{dim} M_{i} \leq 2$ for any $i$. If $\left(g_{i}, f_{i}\right)$ is a homogeneous pair of degree $\alpha$ on $M_{i},(g, f)=\left(g_{1}+\cdots+g_{k}, f_{1}+\right.$ $\left.\cdots+f_{k}\right)$ is a homogeneous pair on $M$. Then we can construct constant sectional curvature pseudo-Riemannian metrics on $M$ by Corollary 3.17. In particular, $g$ is flat.

Now recall the pseudo-Riemannian metric $\hat{g}$ defined in (3.8). Since $\hat{g}_{l}=$ $(1-\alpha) g_{l}, \hat{g}_{l}$ has constant sectional curvature if $g_{l}$ does. In particular, we can further obtain the following in addition to Corollary 3.14

Corollary 3.19. Let $(g, f)$ be a homogeneous pair of degree $\alpha \in \mathbb{R}-\{0,1\}$. Suppose that $g$ is flat. Then the following holds.

- When $\alpha<1$,

- for $C \in \mathbb{R}$ such that $\alpha C>0$, set

$$
v(r)=\frac{\alpha}{4(1-\alpha) C\left(\cosh \left(\frac{1}{2 \sqrt{1-\alpha}}\left(\log r+C_{2}\right)\right)\right)^{2}} \quad\left(C_{2} \in \mathbb{R}\right) .
$$

Then $(v \circ f) \hat{g}$ is a pseudo-Riemannian metric on $M$ which has constant sectional curvature $C$. 
- The pseudo-Riemannian metric $f^{ \pm \frac{1}{\sqrt{1-\alpha}}} \hat{g}$ is flat.

- When $\alpha>1$, set for $C<0$

$$
v(r)=\frac{\alpha}{4(1-\alpha) C\left(\sin \left(\frac{1}{2 \sqrt{\alpha-1}}\left(\log r+C_{2}\right)\right)\right)^{2}} \quad\left(C_{2} \in \mathbb{R}\right) .
$$

Then $(v \circ f) \hat{g}$ is a pseudo-Riemannian metric which has constant sectional curvature $C$ defined on $M-\bigcup_{N \in \mathbb{Z}} f^{-1}\left(\exp \left(2 N \sqrt{\alpha-1} \pi-C_{2}\right)\right)$.

Proof. Since $g$ is flat, we have $K^{g_{l}}=\frac{\alpha}{4 l}$ by Lemma $3.13(2)$. Since $\hat{g}_{l}=(1-\alpha) g_{l}$, it follows that $K^{\hat{g}_{l}}=\frac{\alpha}{4(1-\alpha) l}$, and hence $\frac{l K^{\hat{g}_{l}}}{\alpha}=\frac{1}{4(1-\alpha)}$. Then by Proposition 3.11, it is straightforward to obtain the statement.

Finally, we give an application of Corollary 3.15

Corollary 3.20. Let $(g, f)$ be a homogeneous pair of degree $\alpha>1$. Suppose further that $K^{g} \geq 0$, and $\hat{g}$ is definite in the sense of Definition $A .1$. Then we have

$$
K^{f^{\beta} \hat{g}} \leq 0 \quad \text { for any } \beta \in \mathbb{R} .
$$

Proof. Since $K^{g} \geq 0$, Lemma 3.13 (1) implies that $K^{g_{l}} \geq \frac{\alpha}{4 l}$. Since $\hat{g}_{l}=$ $(1-\alpha) g_{l}$, it follows that $l K^{\hat{g}_{l}}=\frac{l K^{g_{l}}}{1-\alpha} \leq \frac{\alpha}{4(1-\alpha)}<0$.

On the other hand, for any $\beta \in \mathbb{R}$, we have $r^{\beta}=w(0,|\beta| / 4,0, r)$, where we use the notation of Definition 2.12. Since $\alpha \cdot|\beta| / 4 \geq 0$, Corollary 3.15] implies that $K^{f^{\beta} \hat{g}} \leq 0$.

\subsection{The geodesics}

If $v(r)=r^{\beta}$, where $\beta \in \mathbb{R}$, we can describe the geodesics of $\left(M, f^{\beta} g\right)$ in terms of those in $\left(M_{l}, g_{l}\right)$ by Proposition 2.18

Proposition 3.21. Let $(g, f)$ be a homogeneous pair of degree $\alpha$. The geodesic $\gamma:(-\epsilon, \epsilon) \rightarrow M$ with the initial position $x_{0} \in M_{l} \subset M$ and the initial velocity $A \in T_{x_{0}} M$ w.r.t. the pseudo-Riemannian metric $f^{\beta} g$, where $\beta \in \mathbb{R}$, is given as follows.

- When $\beta \neq-1$,

$$
\gamma(t)=m\left(\mu(\beta, t)^{\frac{1}{\alpha(\beta+1)}}, \hat{y}_{l}\left(\int_{0}^{t} \frac{d \tau}{\mu(\beta, \tau)}\right)\right),
$$

where

$$
\mu(\beta, t)=1+\frac{d f(A)}{l}(\beta+1) t+\frac{\alpha}{4 l} g(A, A)(\beta+1)^{2} t^{2}
$$

and $\hat{y}_{l}(s)$ is the geodesic in $\left(M_{l}, g_{l}\right)$ with the initial position $x_{0} \in M_{l}$ and the initial velocity $A-\frac{d f(A)}{\alpha l} P \in T_{x_{0}} M_{l}$, the $T_{x_{0}} M_{l}$ component of $A$ in the decomposition (3.6).

- When $\beta=-1$,

$$
\gamma(t)=m\left(e^{\frac{d f(A)}{\alpha l} t}, \hat{y}_{l}(t)\right)
$$



2.19

Note that the integral $\int_{0}^{t} \frac{d \tau}{\mu(\beta, \tau)}$ can be explicitly computed as in Remark

Proof. By Theorem 3.3, the geodesic $\gamma(t)$ is given by

$$
\gamma(t)=\psi(r(t), y(t))=m\left(\left(\frac{r(t)}{l}\right)^{1 / \alpha}, y(t)\right)
$$

where $(r(t), y(t))$ is a geodesic of $\left(\mathbb{R}_{>0} \times M_{l}, \frac{r^{\beta-1}}{\alpha} d r^{2}+\frac{r^{\beta+1}}{l} g_{l}\right)$ with the initial position $\psi^{-1}\left(x_{0}\right)$ and the initial velocity $\left(d \psi^{-1}\right)_{x_{0}}(A)$. By (3.7), we see that

$$
\psi^{-1}\left(x_{0}\right)=\left(l, x_{0}\right), \quad\left(d \psi^{-1}\right)_{x_{0}}(A)=\left(d f(A), A-\frac{d f(A)}{\alpha l} P\right) .
$$

Since the Levi-Civita connection is invariant under the scalar multiplication of a pseudo-Riemannian metric, $(r(t), y(t))$ is a geodesic of $\frac{l r^{\beta-1}}{\alpha} d r^{2}+r^{\beta+1} g_{l}$, which is of the form (2.18) if we set $k=\frac{l}{\alpha}, C_{0}=\beta+1$ and $\left(Y, g_{Y}\right)=\left(M_{l}, g_{l}\right)$. Then the geodesic $(r(t), y(t))$ is given by Proposition 2.18. Since

$$
\begin{array}{rl}
g\left(A-\frac{d f(A)}{\alpha l} P, A-\frac{d f(A)}{\alpha l} P\right) & =g(A, A)-2 \frac{d f(A)}{\alpha l} g(A, P)+\left(\frac{d f(A)}{\alpha l}\right)^{2} g(P, P) \\
\stackrel{3.3, \sqrt[3.5]{=}}{g} & g(A, A)-2 \frac{d f(A)}{\alpha l} \cdot d f(A)+\left(\frac{d f(A)}{\alpha l}\right)^{2} \cdot \alpha l \\
& =g(A, A)-\frac{1}{\alpha l}(d f(A))^{2},
\end{array}
$$

$F$ in Proposition 2.18 is given by

$$
F=\frac{1}{4}\left(\left(\frac{d f(A)}{l}\right)^{2}+\frac{\alpha}{l}\left(g(A, A)-\frac{1}{\alpha l}(d f(A))^{2}\right)\right)=\frac{\alpha}{4 l} g(A, A) .
$$

Hence the proof is completed.

Corollary 2.20 implies the geodesically convexity or concavity of $f$ in the following cases.

Proposition 3.22. Let $(g, f)$ be a homogeneous pair of degree $\alpha$.

(1) The function $f$ is geodesically convex w.r.t. $f^{\beta} g$ if one of the following condition holds.

- $\beta=-1$.

- $-1<\beta \leq 1$ and $\alpha g_{l}$ is positive definite.

- $\beta<-1$ and $\alpha g_{l}$ is negative definite.

(2) The function $f$ is geodesically concave w.r.t. $f^{\beta} g$ if $\beta \geq 1$ and $\alpha g_{l}$ is negative definite. 
Proof. By Theorem 3.3, any geodesic $\gamma$ w.r.t. $f^{\beta} g$ is of the form

$$
\gamma(t)=\psi(r(t), y(t))=m\left(\left(\frac{r(t)}{l}\right)^{1 / \alpha}, y(t)\right),
$$

where $(r(t), y(t))$ is a geodesic of $\left(\mathbb{R}_{>0} \times M_{l}, \frac{l r^{\beta-1}}{\alpha} d r^{2}+r^{\beta+1} g_{l}\right)$. Then we see that

$$
f(\gamma(t))=\frac{r(t)}{l} f(y(t))=r(t)
$$

where we use (3.2) and the fact that $y(t) \in M_{l}$. Then (1) and (2) hold from Corollary 2.20.

\subsection{The metric completion}

Let $(g, f)$ be a homogeneous pair. Use the notation of Theorem 3.3 , In this subsection, we assume the following.

- The pseudo-Riemannian metric $g$ is positive definite.

- The pseudometric $d_{g}$ induced from $g$ is a metric. (This is always true when $M$ is finite dimensional. In the infinite dimensional case, there are examples of a Riemannian metric whose induced pseudometric is identically zero $([\mathrm{MM}])$. )

Then we study the metric completion of $M$ w.r.t. $d_{(v \circ f) g}$, where $d_{(v \circ f) g}$ is the metric induced from a Riemannian metric $(v \circ f) g$ for a function $v: \mathbb{R}_{>0} \rightarrow \mathbb{R}_{>0}$.

Fixing $R_{0} \in \mathbb{R}_{>0}$, define a strictly increasing function $\hat{T}: \mathbb{R}_{>0} \rightarrow \mathbb{R}$ by

$$
\hat{T}(r)=\int_{R_{0}}^{r} \sqrt{\frac{v(q)}{q}} d q
$$

and set

$$
\hat{T}_{0}=\lim _{r \rightarrow 0} \hat{T}(r) \in \mathbb{R} \cup\{-\infty\}, \quad \hat{T}_{\infty}=\lim _{r \rightarrow \infty} \hat{T}(r) \in \mathbb{R} \cup\{\infty\} .
$$

Then we obtain the following by Remark 3.6 and Theorem 2.27

Theorem 3.23. The metric completion $\bar{M}$ of $M$ w.r.t. the metric $d_{(v \circ f) g}$ induced from the Riemannian metric $(v \circ f) g$ is homeomorphic to the following.

(1) If $\hat{T}_{0}=-\infty$ and $\hat{T}_{\infty}=\infty$,

$$
\mathbb{R}_{>0} \times \overline{M_{l}} \quad \text { with the product topology } .
$$

(2) If $\hat{T}_{0} \in \mathbb{R}, \hat{T}_{\infty}=\infty$ and $\lim _{r \rightarrow 0} r v(r)=0$,

$$
\left(\{0\} \cup \mathbb{R}_{>0}\right) \times \overline{M_{l}} /\left(\{0\} \times \overline{M_{l}}\right)=\left(\mathbb{R}_{>0} \times \overline{M_{l}}\right) \cup\{*\}
$$

with the topology $\mathcal{O}_{0}$ given below.

(3) If $\hat{T}_{0}=-\infty, \hat{T}_{\infty} \in \mathbb{R}$ and $\lim _{r \rightarrow \infty} r v(r)=0$,

$$
\left(\mathbb{R}_{>0} \cup\{\infty\}\right) \times \overline{M_{l}} /\left(\{\infty\} \times \overline{M_{l}}\right)=\left(\mathbb{R}_{>0} \times \overline{M_{l}}\right) \cup\{*\}
$$

with the topology $\mathcal{O}_{\infty}$ given below. 
(4) If $\hat{T}_{0} \in \mathbb{R}, \hat{T}_{\infty} \in \mathbb{R}, \lim _{r \rightarrow 0} r v(r)=0$ and $\lim _{r \rightarrow \infty} r v(r)=0$,

$$
\left(\{0\} \cup \mathbb{R}_{>0} \cup\{\infty\}\right) \times \overline{M_{l}} /\left(\{0, \infty\} \times \overline{M_{l}}\right)=\left(\mathbb{R}_{>0} \times \overline{M_{l}}\right) \cup\{*\} \cup\{*\}
$$

with the topology $\mathcal{O}_{0, \infty}$ given below.

Here, $\overline{M_{l}}$ is the metric completion of $M_{l}$ w.r.t. the metric induced from $g_{l}$.

Let $\pi_{0}:\left(\{0\} \cup \mathbb{R}_{>0}\right) \times \overline{M_{l}} \rightarrow\left(\{0\} \cup \mathbb{R}_{>0}\right) \times \overline{M_{l}} /\left(\{0\} \times \overline{M_{l}}\right)$ be the projection. Set $*_{0}=\pi_{0}\left(\{0\} \times \overline{M_{l}}\right)$. The topology $\mathcal{O}_{0}$ is defined by the fundamental system of neighborhoods $\mathcal{U}(x)$ given below. If $x \neq *_{0}, \mathcal{U}(x)$ consists of $\epsilon$-balls centered at $x$ for $\epsilon>0$ w.r.t. the product metric. If $x=*_{0}$,

$$
\mathcal{U}\left(*_{0}\right)=\left\{\pi_{0}\left([0, \epsilon) \times \overline{M_{l}}\right) \mid \epsilon>0\right\} .
$$

Let $\pi_{\infty}:\left(\mathbb{R}_{>0} \cup\{\infty\}\right) \times \overline{M_{l}} \rightarrow\left(\mathbb{R}_{>0} \cup\{\infty\}\right) \times \overline{M_{l}} /\left(\{\infty\} \times \overline{M_{l}}\right)$ be the projection. Set $*_{\infty}=\pi_{\infty}\left(\{\infty\} \times \overline{M_{l}}\right)$. The topology $\mathcal{O}_{\infty}$ is defined by the fundamental system of neighborhoods $\mathcal{U}(x)$ given below. If $x \neq *_{\infty}, \mathcal{U}(x)$ consists of $\epsilon$-balls centered at $x$ for $\epsilon>0$ w.r.t. the product metric. If $x=*_{\infty}$, we set $\mathcal{U}\left(*_{\infty}\right)=\left\{\pi_{\infty}\left((R, \infty] \times \overline{M_{l}}\right) \mid R>0\right\}$. The topology $\mathcal{O}_{0, \infty}$ is similarly defined by setting the fundamental systems of neighborhoods as above.

Remark 3.24. By Remark 3.4, $\overline{M_{l_{1}}}$ and $\overline{M_{l_{2}}}$ are isometric for $l_{1}, l_{2}>0$. Thus Theorem 3.23 is independent of $l$.

Roughly speaking, the metric completion is the cylinder of $\overline{M_{l}}$ in the case (1), the cone (with the apex) of $\overline{M_{l}}$ in the cases (2) and (3), and the suspension of $\overline{M_{l}}$ in the case (4). In general, the topologies $\mathcal{O}_{0}, \mathcal{O}_{\infty}$ and $\mathcal{O}_{0, \infty}$ are weaker than the quotient topologies. If $\overline{M_{l}}$ is compact, they agree with the quotient topologies. In particular, in the case (4), the metric completion $\bar{M}$ is compact if $\overline{M_{l}}$ is compact.

By Proposition 2.30, we also obtain the following.

Proposition 3.25. Use the notation of Definition A.2. The map

$$
\overline{M_{l}} \rightarrow\left\{\left[x_{k}\right] \in \bar{M} \mid \lim _{k \rightarrow \infty} f\left(x_{k}\right)=l\right\}, \quad\left[y_{k}\right] \mapsto\left[y_{k}\right]
$$

is homeomorphic.

Proof. By Proposition 2.30, the map

$$
\overline{M_{l}} \rightarrow\left\{\left[\left(r_{k}, y_{k}\right)\right] \in \overline{\mathbb{R}_{>0} \times M_{l}} \mid \lim _{k \rightarrow \infty} r_{k}=l\right\}, \quad\left[y_{k}\right] \mapsto\left[\left(l, y_{k}\right)\right]
$$

is homeomorphic. Since $\psi$ in (3.4) is isometric, the map

$$
\overline{\mathbb{R}_{>0} \times M_{l}} \rightarrow \bar{M}, \quad\left[\left(r_{k}, y_{k}\right)\right] \mapsto\left[\psi\left(r_{k}, y_{k}\right)\right]
$$

is isometric. Since $r_{k}=f\left(\psi\left(r_{k}, y_{k}\right)\right)$ and $\psi\left(l, y_{k}\right)=y_{k}$, the proof is completed.

Remark 3.26. Thus if we know $\bar{M}$, we see $\overline{M_{l}}$. In particular, by Theorem 3.23. if we know $\overline{\left(M, d_{(v \circ f) g}\right)}$, the metric completion of $M$ w.r.t. $d_{(v \circ f) g}$, for one $v$, we obtain $\overline{\left(M, d_{(\tilde{v} \circ f) g}\right)}$ for $\tilde{v}$ satisfying one of four assumptions in Theorem 3.23 . 


\section{Pseudo-Hessian manifolds}

Theorem 3.3 applies to many important classes of pseudo-Riemannian manifolds. One of them is the following class, which includes a class of pseudo-Hessian manifolds satisfying the conditions (4.1) (4.3).

Proposition 4.1. Let $M$ be a manifold admitting a torsion-free connection $D$, a function $f: M \rightarrow \mathbb{R}_{>0}$ such that $h=D d f$ is a pseudo-Riemannian metric (If $D$ is flat, $h$ is called a pseudo-Hessian metric), and a free $\mathbb{R}_{>0}$-action $m: \mathbb{R}_{>0} \times M \rightarrow M$. Set $m_{\lambda}=m(\lambda, \cdot)$ for $\lambda>0$. Suppose the following.

- The function $f: M \rightarrow \mathbb{R}_{>0}$ is homogeneous of degree $\alpha \in \mathbb{R}$ :

$$
m_{\lambda}^{*} f=\lambda^{\alpha} f \quad \text { for any } \lambda>0 .
$$

- The action of $\mathbb{R}_{>0}$ preserves $D$ : That is,

$$
D_{\left(m_{\lambda}\right)_{*} A}\left(\left(m_{\lambda}\right)_{*} B\right)=\left(m_{\lambda}\right)_{*}\left(D_{A} B\right)
$$

for any $\lambda>0$ and vector fields $A, B \in \mathfrak{X}(M)$ (cf. [KN, Chapter VI, Proposition 1.4]).

- For a vector field $P \in \mathfrak{X}(M)$ generated by the $\mathbb{R}_{>0}$-action, we have

$$
D_{A} P=A \quad \text { for any } A \in T M .
$$

Then we have $\alpha \neq 0,1$. Moreover, the pairs $(D d f /(\alpha-1), f)$ and $(-f D d \log f, f)$ are homogeneous pairs of degree $\alpha$. In particular, we can apply Theorem 3.3 and we have isometries

$$
\begin{aligned}
(M,(v \circ f) D d f) & \cong\left(\mathbb{R}_{>0} \times M_{l}, \frac{\alpha-1}{\alpha} \cdot \frac{v(r)}{r} d r^{2}+\frac{r v(r)}{l} h_{l}\right), \\
(M,-(v \circ f) D d(\log f)) & \cong\left(\mathbb{R}_{>0} \times M_{l}, \frac{1}{\alpha} \cdot \frac{v(r)}{r^{2}} d r^{2}-\frac{v(r)}{l} h_{l}\right)
\end{aligned}
$$

for any function $v: \mathbb{R}_{>0} \rightarrow \mathbb{R}_{>0}$. Here, $h_{l}$ is the induced pseudo-Riemannian metric on $M_{l}=f^{-1}(l) \subset M$ from $h=D d f$.

Remark 4.2. If we set $g=D d f /(\alpha-1)$, the equation 4.7$)$ implies that $\hat{g}=$ $-f D d \log f$, where $\hat{g}$ is defined in (3.8). In particular, we can apply Corollaries 3.19 and 3.20 to $-f D d \log f$.

Remark 4.3. We can also prove the similar splitting for a pseudo-Riemannian metric $(v \circ f) D d(u \circ f)$ for some $u: \mathbb{R}_{>0} \rightarrow \mathbb{R}$, though $(\operatorname{Dd}(u \circ f), f)$ is not a homogeneous pair in general. That is,

(1) if $\frac{d u}{d r}(r) \neq 0$ and $\frac{d \tilde{u}}{d r}(r) \neq 0$, where we set $\tilde{u}(r)=\alpha r u^{\prime}(r)-u(r), D d(u \circ f)$ is a pseudo-Riemannian metric on $M$.

(2) The map 3.4) gives an isometry between $\left(\mathbb{R}_{>0} \times M_{l}, \frac{\tilde{u}^{\prime}(r) v(r)}{\alpha r} d r^{2}+\frac{r u^{\prime}(r) v(r)}{l} h_{l}\right)$ and $(M,(v \circ f) D d(u \circ f))$.

However, since we do not know examples other than $u(r)=r$ or $\log r$, we omit the proof. We can prove this in the same way as Theorem 3.3 . 
Remark 4.4. Proposition 4.1 generalizes [Loftin, Theorem 1] and [Totaro, Lemmas 2.1 and 2.4]. Indeed, (4.2) and (4.3) are satisfied when $M \subset \mathbb{R}^{n}$ is a cone, $D$ is the canonical flat connection, and the $\mathbb{R}_{>0}$-action is the canonical one.

By setting $v(r)=1, l=1$ and $r=s^{2}$ in 4.4), we see that ( $\left.M, D d f\right)$ is isometric to $\left(\mathbb{R}_{>0} \times M_{1}, \frac{4(\alpha-1)}{\alpha} d s^{2}+s^{2} h_{1}\right)$, which is [Totaro, Lemma 2.1].

Similarly, by setting $v(r)=1, l=1, \alpha>0$ and $r=e^{\sqrt{\alpha} t}$ in $\sqrt{4.4)}$, we see that $(M,-D d(\log f))$ is isometric to $\left(\mathbb{R} \times M_{1}, d t^{2}+\left(-h_{1}\right)\right)$. This is Totaro, Lemma 2.4], which is equivalent to [Loftin, Theorem 1].

Proof of Proposition 4.1. Since the equation (4.1) is the same as (3.2), we have $d f(P)=\alpha f$ by (3.5). Then differentiating $d f(P)=\alpha f$, it follows that

$$
d(d f(P))=(D d f)(P)+d f(D P) \stackrel{4.3}{=}(D d f)(P)+d f=\alpha d f .
$$

Hence we have

$$
h(P, \cdot)=(\alpha-1) d f, \quad h(P, P)=\alpha(\alpha-1) f .
$$

Thus we see that $\alpha \neq 0,1$ so that $h=D d f$ is a pseudo-Riemannian metric. Since the $\mathbb{R}_{>0}$-action preserves the connection $D$, we have

$$
D_{\left(m_{\lambda-1}\right)_{*} A}\left(m_{\lambda}^{*} \alpha\right)=m_{\lambda}^{*}\left(D_{A} \alpha\right)
$$

for $\lambda \in \mathbb{R}_{>0}$, a vector field $A \in \mathfrak{X}(M)$ and a 1-form $\alpha \in \Omega^{1}(M)$. Replacing $A$ with $\left(m_{\lambda}\right)_{*} A$, we see that $D\left(m_{\lambda}^{*} \alpha\right)=m_{\lambda}^{*}(D \alpha)$. Thus we obtain

$$
m_{\lambda}^{*} h=m_{\lambda}^{*}(D d f)=D\left(m_{\lambda}^{*} d f\right) \stackrel{4.1}{=} \lambda^{\alpha} D d f=\lambda^{\alpha} h .
$$

Hence (4.6), (4.1) and (4.5) imply that $(h /(\alpha-1), f)=(D d f /(\alpha-1), f)$ is a homogeneous pair of degree $\alpha$. Then by Theorem 3.3, we have an isometry

$$
\left(M, \frac{(v \circ f) D d f}{\alpha-1}\right) \cong\left(\mathbb{R}_{>0} \times M_{l}, v(r)\left(\frac{1}{\alpha r} d r^{2}+\frac{r}{l} \cdot \frac{h_{l}}{\alpha-1}\right)\right),
$$

which implies the first equation of (4.4).

Similarly, we have

$$
m_{\lambda}^{*} D d(\log f)=D\left(m_{\lambda}^{*} d(\log f)\right)=D d(\log f) .
$$

Since

$$
D d(\log f)=D\left(\frac{d f}{f}\right)=\frac{D d f}{f}-\frac{d f \otimes d f}{f^{2}}
$$

it follows that

$$
\operatorname{Dd}(\log f)(P, \cdot)=\frac{(\alpha-1) d f}{f}-\frac{\alpha d f}{f}=-\frac{d f}{f}
$$

by the equation $d f(P)=\alpha f$ and (4.5). Then we see that $(-f D d(\log f), f)$ is a homogeneous pair of degree $\alpha$. Since $\left.(-f D d(\log f))\right|_{M_{l}}=-h_{l}$ by (4.7), Theorem 3.3 implies an isometry

$$
(M,-(v \circ f) f D d(\log f)) \cong\left(\mathbb{R}_{>0} \times M_{l}, v(r)\left(\frac{1}{\alpha r} d r^{2}+\frac{r}{l}\left(-h_{l}\right)\right)\right),
$$

Then replacing $v(r)$ with $v(r) / r$, we obtain the second equation of (4.4). 


\section{$5 \quad$ Examples}

In this section, we give examples to which we can apply results obtained in previous sections.

\subsection{Manifolds with flat Hessian metrics}

In this subsection, we give examples of manifolds with flat Hessian metrics. We can apply (1)-(4) in Section 1 to these examples.

\subsubsection{Cones in $\mathbb{R}^{n}$}

Many flat Hessian metrics are constructed on cones in $\mathbb{R}^{n}$. Let $D$ be the standard flat connection on $\mathbb{R}^{n}$. It is easy to see that $D$ satisfies (4.2) and (4.3) w.r.t. the canonical $\mathbb{R}_{>0}$-action. We give examples of a function $f: \mathbb{R}^{n} \rightarrow \mathbb{R}$ such that $D d f$ is flat on a cone in $\mathbb{R}^{n}$ where the Hessian of $f$ is positive definite.

- $f\left(x_{1}, \cdots, x_{n}\right)=x_{1}^{2}+\cdots+x_{n}^{2}$,

- $f\left(x_{1}, \cdots, x_{n}\right)=f_{1}\left(x_{1}, x_{2}\right)+f_{2}\left(x_{3}, x_{4}\right)+\cdots$, where $f_{1}, f_{2}, \cdots$ are homogeneous functions of two variables of the same degree such that the Hessian matrices are positive definite.

- $n=3$ and $f(x, y, z)=x^{6}+y^{6}+z^{6}-10\left(x^{3} y^{3}+y^{3} z^{3}+z^{3} x^{3}\right)$, which is called the Maschke sextic.

The first $f$ is the most standard example. The flatness of $D d f$ for the second $f$ is first proved by [Totaro, Section 6], which also follows from Remark 3.18, That for the third $f$ is proved by Dubrovin, Corollary 5.9 and Example 3].

\subsection{Manifolds with pseudo-Hessian metrics}

In this subsection, we give examples of manifolds with pseudo-Hessian metrics. We can apply (1), (3) and (4) in Section 1 to these examples.

\subsubsection{Regular convex cones}

An open convex cone $\Omega \subset \mathbb{R}^{n}$ not containing full straight lines is called a regular convex cone. The study of regular convex cones is considered to be the origin of the geometry of Hessian structures ([Shima, Section 4]). Let $\left(\mathbb{R}^{n}\right)^{*}$ be the dual space of $\mathbb{R}^{n}$. The dual cone $\Omega^{*} \subset\left(\mathbb{R}^{n}\right)^{*}$ is defined by

$$
\Omega^{*}=\left\{y^{*} \in\left(\mathbb{R}^{n}\right)^{*} \mid\left\langle x, y^{*}\right\rangle>0 \text { for any } x \in \bar{\Omega}-\{0\}\right\},
$$

where $\langle\cdot, \cdot\rangle$ is the canonical pairing of $\mathbb{R}^{n}$ and $\left(\mathbb{R}^{n}\right)^{*}$ and $\bar{\Omega}$ is the closure of $\Omega$. The characteristic function $f: \Omega \rightarrow \mathbb{R}$ is given by

$$
f(x)=\int_{\Omega^{*}} e^{-\left\langle x, x^{*}\right\rangle} d x^{*},
$$

where $d x^{*}$ is the canonical volume form on $\left(\mathbb{R}^{n}\right)^{*}$. This function $f$ is homogeneous of degree $-n$, which follows [Shima, (4.2)], and $D d(\log f)$ defines a Riemannian metric on $\Omega$, where $D$ is the standard flat connection on the Euclidean space ([Shima, Proposition 4.5]). 


\subsubsection{The Kähler cone}

Let $M$ be a compact Kähler manifold of $\operatorname{dim}_{\mathbb{C}} M=n$. Let

$$
\mathcal{K}=\left\{\omega \in H^{1,1}(M, \mathbb{R}) \mid \omega \text { contains a Kähler metric }\right\}
$$

be the Kähler cone of $M$, which is an open cone in $H^{1,1}(M, \mathbb{R})$. Define a function $f: \mathcal{K} \rightarrow \mathbb{R}$ by

$$
f(\omega)=\operatorname{Vol}(\omega)=\int_{M} \frac{\omega^{n}}{n !},
$$

which is homogeneous of degree $n$ w.r.t. the canonical $\mathbb{R}_{>0}$-action on $\mathcal{K}$. Then it is known that $g=-D d(\log f)$ is a Riemannian metric on $\mathcal{K}$, where $D$ is the standard flat connection on $\mathcal{K}$ (Magnússon, Proposition 1.1]). The Riemannian metric $g$ is complete if and only if $\mathcal{K}$ is a connected component of the volume cone $\left\{\omega \in H^{1,1}(M, \mathbb{R}) \mid \int_{M} \omega^{n}>0\right\}$ (Magnússon, Proposition 4.4]).

The level sets $\mathcal{K}_{l}=f^{-1}(l) \subset \mathcal{K}$, where $l>0$, with the induced Riemannian metric $g_{l}$ was studied in Huybrechts, Wilson, TW. Wilson explicitly computed the curvature tensor and the geodesics of $g_{l}$. He conjectured that when $M$ is a Calabi-Yau manifold, $\mathcal{K}_{l}$ should have non-positive sectional curvatures, at least in the large Kähler structure limit, considering the correspondence to the WeilPetersson metric on the complex moduli space under mirror symmetry. Now, there are some counterexamples in Totaro, TW].

When $h^{1,1}=\operatorname{dim} H^{1,1}(X, \mathbb{R})=2$ or $M$ is hyperkähler, $g_{l}$ has constant negative sectional curvature. See Wilson, p.631 and Example 1].

\subsubsection{The $G_{2}$ moduli space}

The exceptional Lie group $G_{2}$ is realized as a stabilizer in $\operatorname{GL}(7, \mathbb{R})$ of a 3 -form $\varphi_{0}$ on $\mathbb{R}^{7}$. The $\mathrm{GL}_{+}(7, \mathbb{R})$-orbit $\mathrm{GL}_{+}(7, \mathbb{R}) \cdot \varphi_{0}$ through $\varphi_{0}$, where $\mathrm{GL}_{+}(7, \mathbb{R})=$ $\{A \in \mathrm{GL}(7, \mathbb{R}) \mid \operatorname{det} A>0\}$, is diffeomorphic to $\mathrm{GL}_{+}(7, \mathbb{R}) / G_{2}$. It has the same dimension as $\Lambda^{3}\left(\mathbb{R}^{7}\right)^{*}$, and hence it is open in $\Lambda^{3}\left(\mathbb{R}^{7}\right)^{*}$. Any $\varphi \in \mathrm{GL}_{+}(7, \mathbb{R}) \cdot \varphi_{0}$ induces the metric $g_{\varphi}$, the volume form $\operatorname{vol}_{\varphi}$ and the Hodge star $*_{\varphi}$ on $\mathbb{R}^{7}$. They are related by

$$
i(u) \varphi \wedge i(v) \varphi \wedge \varphi=6 g_{\varphi}(u, v) \operatorname{vol}_{\varphi}, \quad \varphi \wedge *_{\varphi} \varphi=7 \operatorname{vol}_{\varphi} \quad \text { for } u, v \in \mathbb{R}^{7} .
$$

Let $M^{7}$ be a 7 -dimensional manifold with a $G_{2}$-structure. That is, the tangent frame bundle is reduced to a $G_{2}$-subbundle. We assume that $M^{7}$ is connected for simplicity. We can define a positive 3 -form, a section of an open subbundle $\Lambda_{+}^{3} T^{*} M^{7}$ of $\Lambda^{3} T^{*} M^{7}$, which is induced from $\mathrm{GL}_{+}(7, \mathbb{R}) \cdot \varphi_{0}$. We denote by $\nabla^{\varphi}$ the Levi-Civita connection of $g_{\varphi}$. Then a Riemannian metric $g$ has holonomy contained in $G_{2}$ if and only if there exists a positive 3 -form such that $\nabla^{\varphi} \varphi=0$ and $g=g_{\varphi}$. A positive 3 -form $\varphi$ satisfying $\nabla^{\varphi} \varphi=0$ is called a torsion-free $G_{2}$-structure.

The holonomy group of $g_{\varphi}$ for a torsion-free $G_{2}$-structure $\varphi$ is determined by the topology of $M^{7}$. It has full holonomy $G_{2}$ if and only if $\pi_{1}\left(M^{7}\right)$ is finite. We call such a manifold irreducible.

Define the moduli space $\mathcal{M}_{G_{2}}$ of torsion-free $G_{2}$-structures by

$$
\mathcal{M}_{G_{2}}=\left\{\varphi \in \Omega_{+}^{3}\left(M^{7}\right) \mid \nabla^{\varphi} \varphi=0\right\} / \operatorname{Diff}_{0}\left(M^{7}\right),
$$


where $\Omega_{+}^{3}\left(M^{7}\right)$ is the space of smooth positive 3 -forms and $\operatorname{Diff}_{0}\left(M^{7}\right)$ is the identity component of the diffeomorphism group.

Suppose that $M^{7}$ is compact. By Joyce2, a map $\mathcal{M}_{G_{2}} \ni[\varphi] \mapsto[\varphi] \in$ $H^{3}\left(M^{7}, \mathbb{R}\right)$ is a local homeomorphism, which implies that $\mathcal{M}_{G_{2}}$ is an affine manifold of dimension $b^{3}=\operatorname{dim} H^{3}\left(M^{7}, \mathbb{R}\right)$. Denote by $D$ the flat connection on $\mathcal{M}_{G_{2}}$ (cf. [KL, Section 3.1]). This satisfies (4.2) and (4.3) w.r.t. the canonical

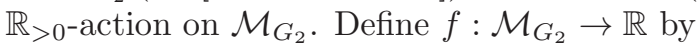

$$
f([\varphi])=3 \operatorname{Vol}(\varphi)=3 \int_{M^{7}} \operatorname{vol}_{\varphi}=\frac{3}{7} \int_{M^{7}} \varphi \wedge * \varphi \varphi,
$$

which is homogeneous of degree $7 / 3$ w.r.t. the canonical $\mathbb{R}_{>0}$-action on $\mathcal{M}_{G_{2}}$. We have three canonical pseudo-Riemannian metrics on $\mathcal{M}_{G_{2}}$ (cf. Hitchin2, Proposition 22], [KL, Theorem 3.5 and Lemma 3.11]).

(1) The tensor $h_{1}=D d f$ is a pseudo-Riemannian metric with signature $(1+$ $\left.b^{1}, b^{3}-1-b^{1}\right)$, where $b^{i}$ is the $i$-th Betti number.

(2) The tensor $h_{2}=-D d(\log f)$ is a pseudo-Riemannian metric with signature $\left(b^{3}-b^{1}, b^{1}\right)$. When $M$ is irreducible, this is positive definite.

(3) By identifying $T_{[\varphi]} \mathcal{M}_{G_{2}}$ with $\mathcal{H}_{\varphi}^{3}$, the space of harmonic 3-forms w.r.t. $g_{\varphi}$, the $L^{2}$-metric on $M$ induces the metric $g_{L^{2}}$ on $\mathcal{M}_{G_{2}}$. When $M$ is irreducible, we have

$$
g_{L^{2}}=-f D d \log f .
$$

By Proposition 4.1, $\left(\frac{3}{4} h_{1}, f\right)$ and $\left(g_{L^{2}}, f\right)$ are homogeneous pairs on $\mathcal{M}_{G_{2}}$. These two Riemannian-metrics are related by $g_{L^{2}}=\widehat{\frac{3}{4} h_{1}}$, by Remark 4.2 By the conformal transformation of $g_{L^{2}}$, we obtain $h_{2}$.

Remark 5.1. As far as the author knows, there are no known examples of a \%dimensional manifold admitting a torsion-free $G_{2}$-structure with $b^{3}=\operatorname{dim} \mathcal{M}_{G_{2}}=$ 2. It would be interesting to construct such examples. It is because the above pseudo-Riemannian metrics are flat by Corollary 3.17, and hence $\mathcal{M}_{G_{2}}$ is expected to have simpler geometric structures, which might be useful to study the general cases.

The Hessian curvature tensor for $h_{2}$ is explicitly given in $[G Y]$. See also Grigorian. The detailed analysis of the curvature of $\mathcal{M}_{G_{2}}$ is also given in KLL

The metric completion of $\mathcal{M}_{G_{2}}$ has not been studied yet. By Theorem 3.23, we see the following.

Corollary 5.2. When $M^{7}$ is irreducible, the metric completion $\overline{\left(\mathcal{M}_{G_{2}}, d_{h_{2}}\right)}$ of $\mathcal{M}_{G_{2}}$ (resp. $\left.\overline{\left(\mathcal{M}_{G_{2}}, d_{g_{L^{2}}}\right)}\right)$ w.r.t. the metric $d_{h_{2}}$ (resp. $d_{g_{L^{2}}}$ ) induced from $h_{2}$ (resp. $\left.g_{L^{2}}\right)$ is homeomorphic to $\mathbb{R}_{>0} \times \overline{\left(\mathcal{M}_{G_{2}}\right)_{l}}\left(\right.$ resp. $\left..\left(\{0\} \cup \mathbb{R}_{>0}\right) \times \overline{\left(\mathcal{M}_{G_{2}}\right)_{l}} /\left(\{0\} \times \overline{M_{l}}\right)\right)$, where $\overline{\left(\mathcal{M}_{G_{2}}\right)_{l}}$ is the metric completion of $\left(\mathcal{M}_{G_{2}}\right)_{l}=f^{-1}(l) \subset \mathcal{M}_{G_{2}}$ w.r.t. the induced Riemannian metric from $h_{2}$.

In particular, $\overline{\left(\mathcal{M}_{G_{2}}, d_{h_{2}}\right)}$ is strictly smaller than $\overline{\left(\mathcal{M}_{G_{2}}, d_{g_{L^{2}}}\right)}$. In other words, $\overline{\left(\mathcal{M}_{G_{2}}, d_{h_{2}}\right)}$ has less degenerate points than $\overline{\left(\mathcal{M}_{G_{2}}, d_{g_{L^{2}}}\right)}$. 
Remark 5.3. The completion of the space of Riemannian metrics is described geometrically in terms of measurable, symmetric, positive semidefinite $(0,2)$ tensor fields (cf. [Clarke3, CR1], Section 5.3.2). We may also expect to describe $\overline{\left(\mathcal{M}_{G_{2}}\right)_{l}}$ geometrically, which is equivalent to describe $\overline{\left(\mathcal{M}_{G_{2}}, d_{h_{2}}\right)}$ or $\overline{\left(\mathcal{M}_{G_{2}}, d_{g_{L^{2}}}\right)}$ geometrically by Theorem 3.23 and Proposition 3.25, but it seems to be difficult.

If we follow the case above, we have to describe geometrically the metric completions of $\Omega_{+}^{3}\left(M^{7}\right)$ and $\widehat{\mathcal{M}}_{G_{2}}=\left\{\varphi \in \Omega_{+}^{3}\left(M^{7}\right) \mid \nabla^{\varphi} \varphi=0\right\}$ to give a geometrical description of $\mathcal{M}_{G_{2}}=\widehat{\mathcal{M}}_{G_{2}} / \operatorname{Diff}_{0}\left(M^{7}\right)$.

We expect that the metric completion of $\Omega_{+}^{3}\left(M^{7}\right)$ w.r.t. the $L^{2}$ metric divided by the volume functional (which corresponds to $h_{2}$ on $\mathcal{M}_{G_{2}}$ ) will be the set of measurable semi-positive $G_{2}$-structures with nonzero finite volume modulo the equivalence relation as in Theorem 5.8. Here, we call the section of the closure of $\Lambda_{+}^{3} T^{*} M^{7}$ "semi-positive".

It will be difficult to determine the metric completion of $\widehat{\mathcal{M}}_{G_{2}}=\{\varphi \in$ $\left.\Omega_{+}^{3}\left(M^{7}\right) \mid \nabla^{\varphi} \varphi=0\right\}$. The related problem is considered in [CR1], where the Calabi-Yau theorem is used in the proof. There is no such analogues in the $G_{2}$ case. For the metric completion of $\mathcal{M}_{G_{2}}$, there will be more problems when taking the quotient by $\operatorname{Diff}_{0}\left(M^{7}\right)$ as in Clarke3, Section 5].

It would also be an interesting question to study the metric completion of the space of closed $G_{2}$-structures $\left\{\varphi \in \Omega_{+}^{3}\left(M^{7}\right) \mid d \varphi=0\right\}$. We might characterize the existence of torsion-free $G_{2}$-structures in terms of the "analytic stability condition" in terms of the Laplacian flow and the metric completion as in [CR1].

\subsubsection{The $\mathrm{SL}(3, \mathbb{C})$ moduli space}

The group $\operatorname{SL}(3, \mathbb{C})$ is realized as a stabilizer in $\operatorname{GL}(6, \mathbb{R})$ of a 3 -form $\psi_{0}=$ $\operatorname{Re}\left(d z^{1} \wedge d z^{2} \wedge d z^{3}\right)$ on $\mathbb{R}^{6}$, where we use holomorphic coordinates $\left(z^{1}, z^{2}, z^{3}\right)$ on $\mathbb{C}^{3} \cong \mathbb{R}^{6}$. The $\mathrm{GL}_{+}(6, \mathbb{R})$-orbit $\mathrm{GL}_{+}(6, \mathbb{R}) \cdot \psi_{0}$ through $\psi_{0}$, where $\mathrm{GL}_{+}(6, \mathbb{R})=$ $\{A \in \mathrm{GL}(6, \mathbb{R}) \mid \operatorname{det} A>0\}$, is diffeomorphic to $\mathrm{GL}_{+}(6, \mathbb{R}) / \mathrm{SL}(3, \mathbb{C})$. It has the same dimension as $\Lambda^{3}\left(\mathbb{R}^{6}\right)^{*}$, and hence it is open in $\Lambda^{3}\left(\mathbb{R}^{6}\right)^{*}$. By Hitchin2, (9), $(10)]$, Any $\psi \in \mathrm{GL}_{+}(6, \mathbb{R}) \cdot \psi_{0}$ induces a complex structure $J_{\psi}$ and a 3 -form $\hat{\psi}$ on $\mathbb{R}^{6}$ such that $\psi+i \hat{\psi}$ is a $(3,0)$-form w.r.t. $J_{\psi}$.

Let $M^{6}$ be a 6 -dimensional manifold with a $\operatorname{SL}(3, \mathbb{C})$-structure. That is, the tangent frame bundle is reduced to a $\mathrm{SL}(3, \mathbb{C})$-subbundle. We can define a positive 3 -form, a section of an open subbundle $\Lambda_{+}^{3} T^{*} M^{6}$ of $\Lambda^{3} T^{*} M^{6}$, which is induced from $\mathrm{GL}_{+}(6, \mathbb{R}) \cdot \psi_{0}$. We call a positive 3 -form $\psi$ torsion-free if $d \psi=d \hat{\psi}=0$.

Define the moduli space $\mathcal{M}_{\mathrm{SL}(3, \mathbb{C})}$ of torsion-free $\mathrm{SL}(3, \mathbb{C})$-structures by

$$
\mathcal{M}_{\mathrm{SL}(3, \mathbb{C})}=\left\{\psi \in \Omega_{+}^{3}\left(M^{6}\right) \mid d \psi=d \hat{\psi}=0\right\} / \operatorname{Diff}_{0}\left(M^{6}\right),
$$

where $\Omega_{+}^{3}\left(M^{6}\right)$ is the space of smooth positive 3 -forms and $\operatorname{Diff}_{0}\left(M^{6}\right)$ is the identity component of the diffeomorphism group.

Suppose that $M^{6}$ is a compact complex 3-manifold with non-vanishing holomorphic 3-form and satisfy the $\partial \bar{\partial}$-lemma (such as a Calabi-Yau manifold). Then by [Hitchin2, Section 6.3], a map $\mathcal{M}_{\mathrm{SL}(3, \mathbb{C})} \ni[\psi] \mapsto[\psi] \in H^{3}\left(M^{6}, \mathbb{R}\right)$ is a local homeomorphism, which implies that $\mathcal{M}_{\mathrm{SL}(3, \mathbb{C})}$ is an affine manifold of dimension $b^{3}\left(M^{6}\right)$. Denote by $D$ the flat connection on $\mathcal{M}_{\mathrm{SL}(3, \mathbb{C})}$. This satisfies (4.2) and (4.3) w.r.t. the canonical $\mathbb{R}_{>0}$-action on $\mathcal{M}_{\mathrm{SL}(3, \mathbb{C})}$. Define 
$f: \mathcal{M}_{\mathrm{SL}(3, \mathbb{C})} \rightarrow \mathbb{R}$ by

$$
f([\psi])=\int_{M^{6}} \psi \wedge \hat{\psi}
$$

which is homogeneous of degree 2 w.r.t. the canonical $\mathbb{R}_{>0}$-action on $\mathcal{M}_{G_{2}}$ by the definition of $\hat{\psi}$ in [Hitchin2, Definition 2]. Then the Hessian Ddf of $f$ defines a pseudometric on $\mathcal{M}_{\mathrm{SL}(3, \mathbb{C})}$. In fact, $\mathcal{M}_{\mathrm{SL}(3, \mathbb{C})}$ admits a more geometric structure. It is known to be a special pseudo-Kähler manifold (Hitchin2, Proposition 17]).

\subsection{Other examples}

In this subsection, we give examples which admit a homogeneous pair but are not known to admit pseudo-Hessian structures. We can also apply (1), (3) and (4) in Section 1 to these examples.

\subsubsection{The Spin(7) moduli space}

The group $\operatorname{Spin}(7)$ is realized as a stabilizer in $\operatorname{GL}(8, \mathbb{R})$ of a 4 -form $\Phi_{0}$ on $W=\mathbb{R}^{8}$. It is known that $\operatorname{Spin}(7) \subset \mathrm{SO}(8)$. The $\mathrm{GL}_{+}(8, \mathbb{R})$-orbit $\mathrm{GL}_{+}(8, \mathbb{R}) \cdot \Phi_{0}$ through $\Phi_{0}$, where $\mathrm{GL}_{+}(8, \mathbb{R})=\{A \in \mathrm{GL}(8, \mathbb{R}) \mid \operatorname{det} A>0\}$, is diffeomorphic to $\mathrm{GL}_{+}(8, \mathbb{R}) / \operatorname{Spin}(7)$. Note that this is not open in $\Lambda^{4} W^{*}$ as in the cases $G_{2}$ and $\operatorname{SL}(3, \mathbb{C})$. Any $\Phi \in \mathrm{GL}_{+}(8, \mathbb{R}) \cdot \Phi_{0}$ induces the metric $g_{\Phi}$, the volume form $\operatorname{vol}_{\Phi}$ and the Hodge star $*_{\Phi}$ on $\mathbb{R}^{8}$. Note that $\Phi$ and $\operatorname{vol}_{\Phi}$ are related by

$$
\Phi \wedge \Phi=14 \operatorname{vol}_{\Phi} .
$$

The group $\operatorname{Spin}(7)$ acts canonically on the space of forms $\Lambda^{*} W^{*}$ on $W$. In particular, $\Lambda^{4} W^{*}$ has the following irreducible decomposition

$$
\Lambda^{4} W^{*}=\Lambda_{1}^{4} W^{*} \oplus \Lambda_{7}^{4} W^{*} \oplus \Lambda_{27}^{4} W^{*} \oplus \Lambda_{35}^{4} W^{*},
$$

where $\Lambda_{k}^{4} W^{*}$ is a $k$-dimensional irreducible representation of $\operatorname{Spin}(7)$. Note that $\Lambda_{1}^{4} W^{*}=\mathbb{R} \Phi_{0}$ and

$$
\Lambda_{1}^{4} W^{*} \oplus \Lambda_{7}^{4} W^{*} \oplus \Lambda_{27}^{4} W^{*}=\Lambda_{+}^{4} W^{*}, \quad \Lambda_{35}^{4} W^{*}=\Lambda_{-}^{4} W^{*},
$$

where $\Lambda_{+}^{4} W^{*}$ (resp. $\left.\Lambda_{-}^{4} W^{*}\right)$ is the space of self-dual (resp. anti-self-dual) 4forms.

Let $M^{8}$ be an 8-dimensional manifold with a $\operatorname{Spin}(7)$-structure, that is, the tangent frame bundle is reduced to a $\operatorname{Spin}(7)$-subbundle. We assume that $M^{8}$ is connected for simplicity. We can define an admissible 4 -form, a section of a $43(=1+7+35)$-dimensional subbundle $\mathcal{A}^{4} M^{8}$ of $\Lambda^{4} T^{*} M^{8}$, which is induced from $\mathrm{GL}_{+}(8, \mathbb{R}) \cdot \Phi_{0}$. We denote by $\nabla^{\Phi}$ the Levi-Civita connection of $g_{\Phi}$. Then a Riemannian metric $g$ has holonomy contained in $\operatorname{Spin}(7)$ if and only if there exists an admissible 4 -form $\Phi$ such that $\nabla^{\Phi} \Phi=0$ and $g=g_{\Phi}$. It is known that $\nabla^{\Phi} \Phi=0$ if and only if $d \Phi=0$. An admissible 4-form $\Phi$ satisfying $d \Phi=0$ is called a torsion-free $\operatorname{Spin}(7)$-structure.

The holonomy group of $g_{\Phi}$ for a torsion-free $\operatorname{Spin}(7)$-structure $\Phi$ is determined by the topology of $M^{8}$. It has full holonomy $\operatorname{Spin}(7)$ if and only if $M^{8}$ is simply connected and the Betti numbers of $M^{8}$ satisfy $b^{3}+b_{+}^{4}=b^{2}+2 b_{-}^{4}+25$. We call such a manifold irreducible. In this case, we have $b_{7}^{4}=0$ (cf. Joyce3, Proposition 10.6.5 and Theorem 10.6.8]). 
On a manifold $M^{8}$ admitting a torsion-free $\operatorname{Spin}(7)$-structure $\Phi$, there is a decomposition of $\Omega^{4}\left(M^{8}\right)$, the space of smooth 4 -forms, induced from (5.1):

$$
\Omega^{4}\left(M^{8}\right)=\Omega_{1}^{4}\left(M^{8}\right) \oplus \Omega_{7}^{4}\left(M^{8}\right) \oplus \Omega_{27}^{4}\left(M^{8}\right) \oplus \Omega_{35}^{4}\left(M^{8}\right),
$$

where we denote by $\Omega_{k}^{4}\left(M^{8}\right)$ the space of smooth sections of $\Lambda_{k}^{4} T^{*} M^{8}$. Set $\left(\mathcal{H}_{k}^{4}\right)_{\Phi}=\left\{\xi \in \Omega_{k}^{4}\left(M^{8}\right) \mid d \xi=d *_{\Phi} \xi=0\right\}$, which is the space of harmonic forms in $\Omega_{k}^{4}\left(M^{8}\right), b_{k}^{4}=\operatorname{dim}\left(\mathcal{H}_{k}^{4}\right)_{\Phi}$ and

$$
\mathcal{H}_{\Phi}=\left(\mathcal{H}_{1}^{4}\right)_{\Phi} \oplus\left(\mathcal{H}_{7}^{4}\right)_{\Phi} \oplus\left(\mathcal{H}_{35}^{4}\right)_{\Phi} .
$$

Define the moduli space $\mathcal{M}_{\operatorname{Spin}(7)}$ of torsion-free $\operatorname{Spin}(7)$-structures by

$$
\begin{aligned}
& \widehat{\mathcal{M}}_{\operatorname{Spin}(7)}=\left\{\Phi \in C^{\infty}\left(\mathcal{A}^{4} M^{8}\right) \mid d \Phi=0\right\}, \\
& \mathcal{M}_{\operatorname{Spin}(7)}=\widehat{\mathcal{M}}_{\operatorname{Spin}(7)} / \operatorname{Diff}_{0}\left(M^{8}\right),
\end{aligned}
$$

where $C^{\infty}\left(\mathcal{A}^{4} M^{8}\right)$ is the space of smooth admissible 4 -forms and $\operatorname{Diff}_{0}\left(M^{8}\right)$ is the identity component of the diffeomorphism group. Let $\pi: \widehat{\mathcal{M}}_{\operatorname{Spin}(7)} \rightarrow$ $\mathcal{M}_{\text {Spin(7) }}$ be the canonical projection.

As far as the author knows, the geometric structures of $\mathcal{M}_{\operatorname{Spin}(7)}$ have not been studied yet. Thus by recalling the result of Joyce1 about the smoothness of $\mathcal{M}_{\operatorname{Spin}(7)}$, we explain two pseudo-Riemannian metrics on $\mathcal{M}_{\operatorname{Spin}(7)}$ in detail.

Suppose that $M^{8}$ is compact. By Joyce1, by fixing any $\Phi \in \widehat{\mathcal{M}}_{\operatorname{Spin}(7)}$, there exist open neighborhoods $\mathcal{U} \subset \mathcal{H}_{\Phi}$ of 0 and $\mathcal{V} \subset \mathcal{M}_{\operatorname{Spin}(7)}$ of $\pi(\Phi)$ and a smooth map $\widehat{\Phi}: \mathcal{U} \rightarrow \widehat{\mathcal{M}}_{\operatorname{Spin}(7)}$ such that $\widehat{\Phi}(0)=\Phi,(d \widehat{\Phi})_{0}(\xi)=\xi$ for any $\xi \in \mathcal{H}_{\Phi}$, and $\pi \circ \widehat{\Phi}: \mathcal{U} \rightarrow \mathcal{V}$ is a homeomorphism. Then we see that $\mathcal{M}_{\operatorname{Spin}(7)}$ is a smooth manifold of dimension $b_{1}^{4}+b_{7}^{4}+b_{35}^{4}$, which is known to be a topological invariant. Thus we have the identification

$$
T_{\pi(\Phi)} \mathcal{M}_{\operatorname{Spin}(7)}=(d \pi)_{\Phi}\left(\mathcal{H}_{\Phi}\right) .
$$

However, $\mathcal{M}_{\operatorname{Spin}(7)}$ is not known to be an affine manifold as in the cases of $G_{2}$ and $\operatorname{SL}(3, \mathbb{C})$.

By (5.4), we can define two canonical pseudo-Riemannian metrics $g_{I}$ and $g_{L^{2}}$ on $\mathcal{M}_{\operatorname{Spin}(7)}$.

(1) For $\Phi \in \widehat{\mathcal{M}}_{\operatorname{Spin}(7)}$ and $\xi, \eta \in \mathcal{H}_{\Phi}$, define

$$
\left(g_{I}\right)_{\pi(\Phi)}\left((d \pi)_{\Phi}(\xi),(d \pi)_{\Phi}(\eta)\right)=\int_{M^{8}} \xi \wedge \eta
$$

which is induced from the intersection form on $H^{4}\left(M^{8}, \mathbb{R}\right)$.

(2) For $\Phi \in \widehat{\mathcal{M}}_{\operatorname{Spin}(7)}$ and $\xi, \eta \in \mathcal{H}_{\Phi}$, define

$$
\left(g_{L^{2}}\right)_{\pi(\Phi)}\left((d \pi)_{\Phi}(\xi),(d \pi)_{\Phi}(\eta)\right)=\int_{M^{8}} \xi \wedge *_{\Phi} \eta,
$$

which is induced from the $L^{2}$-metric on $M^{8}$, and hence $g_{L^{2}}$ is positive definite. 
Lemma 5.4. The pseudo-Riemannian metrics $g_{I}$ and $g_{L^{2}}$ are well-defined.

Proof. Take any $\Phi \in \widehat{\mathcal{M}}_{\text {Spin(7) }}$ and $\theta \in \operatorname{Diff}_{0}\left(M^{8}\right)$. The Riemannian metric $g_{\Phi}$ induced from $\Phi$ is given explicitly in Karigiannis, Theorem 4.3.5], which implies that

$$
g_{\theta^{*} \Phi}=\theta^{*} g_{\Phi} .
$$

Then we easily see that the induced Hodge stars are related by

$$
*_{\theta}{ }^{*}=\theta^{*} *_{\Phi}\left(\theta^{-1}\right)^{*} \text {. }
$$

Then for any $\xi \in \mathcal{H}_{\Phi}$, we have $\theta^{*} \xi \in \mathcal{H}_{\theta^{*} \Phi}$. The equation $\pi=\pi \circ \theta^{*}$ implies that $(d \pi)_{\Phi}(\xi)=(d \pi)_{\theta^{*} \Phi}\left(\theta^{*} \xi\right)$. Thus we only have to prove

$$
\int_{M^{8}} \theta^{*} \xi \wedge \theta^{*} \eta=\int_{M^{8}} \xi \wedge \eta, \quad \text { and } \quad \int_{M^{8}} \theta^{*} \xi \wedge *_{\theta^{*} \Phi} \theta^{*} \eta=\int_{M^{8}} \xi \wedge *_{\Phi} \eta
$$

for any $\xi, \eta \in \mathcal{H}_{\Phi}$. These equations follow from $\theta \in \operatorname{Diff}_{0}\left(M^{8}\right)$ and (5.7).

If we decompose $\xi=\xi_{1}+\xi_{7}+\xi_{35}$ and $\eta=\eta_{1}+\eta_{7}+\eta_{35}$ following (5.3), the equation (5.2) implies that

$$
\begin{aligned}
& \left(g_{I}\right)_{\pi(\Phi)}\left((d \pi)_{\Phi}(\xi),(d \pi)_{\Phi}(\eta)\right)=\int_{M^{8}} \xi_{1} \wedge *_{\Phi} \eta_{1}+\int_{M^{8}} \xi_{7} \wedge *_{\Phi} \eta_{7}-\int_{M^{8}} \xi_{35} \wedge *_{\Phi} \eta_{35}, \\
& \left(g_{L^{2}}\right)_{\pi(\Phi)}\left((d \pi)_{\Phi}(\xi),(d \pi)_{\Phi}(\eta)\right)=\int_{M^{8}} \xi_{1} \wedge \eta_{1}+\int_{M^{8}} \xi_{7} \wedge \eta_{7}-\int_{M^{8}} \xi_{35} \wedge \eta_{35} .
\end{aligned}
$$

In particular, (5.8) implies that $g_{I}$ has signature $\left(1+b_{7}^{4}, b_{35}^{4}\right)$ and it is Lorentzian if $M^{8}$ is irreducible.

Define a function $f: \mathcal{M}_{\mathrm{Spin}(7)} \rightarrow \mathbb{R}$ by

$$
f(\pi(\Phi))=7 \operatorname{Vol}(\Phi)=7 \int_{M^{8}} \operatorname{vol}_{\Phi}=\frac{1}{2} \int_{M^{8}} \Phi \wedge \Phi,
$$

which is homogeneous of degree 2 w.r.t. the canonical $\mathbb{R}_{>0}$-action on $\mathcal{M}_{\operatorname{Spin}(7)}$.

Proposition 5.5. The pairs $\left(g_{I}, f\right)$ and $\left(g_{L^{2}}, f\right)$ are homogeneous pairs of degree 2 w.r.t. the canonical $\mathbb{R}_{>0}$-action on $\mathcal{M}_{\mathrm{Spin}(7)}$.

Proof. By (5.5) and (5.9), we see that (3.1) and (3.2) are satisfied for $\alpha=2$. The vector field $P$ generated by the canonical $\mathbb{R}_{>0}$-action on $\mathcal{M}_{\operatorname{Spin}(7)}$ is given by

$$
P_{\pi(\Phi)}=(d \pi)_{\Phi}(\Phi) .
$$

Then by (5.8), we have for any $\eta \in \mathcal{H}_{\Phi}$,

$$
\left(g_{I}\right)_{\pi(\Phi)}\left(P_{\pi(\Phi)},(d \pi)_{\Phi}(\eta)\right)=\int_{M^{8}} \Phi \wedge *_{\Phi} \eta_{1}=\left(g_{L^{2}}\right)_{\pi(\Phi)}\left(P_{\pi(\Phi)},(d \pi)_{\Phi}(\eta)\right) .
$$

On the other hand, we compute

$$
\begin{aligned}
(d f)_{\pi(\Phi)}\left((d \pi)_{\Phi}(\eta)\right) & =d(f \circ \pi \circ \widehat{\Phi})_{0}\left(\left.\frac{d}{d t}(t \eta)\right|_{t=0}\right) \\
& =\left.\frac{1}{2} \frac{d}{d t} \int_{M^{8}} \widehat{\Phi}(t \eta) \wedge \widehat{\Phi}(t \eta)\right|_{t=0} \\
& =\int_{M^{8}} \eta \wedge \Phi=\left(g_{I}\right)_{\pi(\Phi)}\left(P_{\pi(\Phi)},(d \pi)_{\Phi}(\eta)\right) .
\end{aligned}
$$


Hence the proof is completed.

Two pseudo-Riemannian metrics $g_{I}$ and $g_{L^{2}}$ are related as follows.

Remark 5.6. If $M^{8}$ is irreducible, $g_{I}$ and $g_{L^{2}}$ are related by $g_{L^{2}}=\widehat{g_{I}}$, where we use the notation in (3.8).

Indeed, take any $\Phi \in \widehat{\mathcal{M}}_{\operatorname{Spin}(7)}$ and $\eta=\eta_{1}+\eta_{35} \in\left(\mathcal{H}_{1}^{4}\right)_{\Phi} \oplus\left(\mathcal{H}_{35}^{4}\right)_{\Phi}$. By the proof of Proposition 5.5, we have $(d f)_{\pi(\Phi)}\left((d \pi)_{\Phi}(\eta)\right)=\langle\eta, \Phi\rangle_{L^{2}}$, where $\langle\cdot, \cdot\rangle$ is the $L^{2}$-metric on the space of differential forms on $M^{8}$ induced from $g_{\Phi}$. Since $\left(\mathcal{H}_{1}^{4}\right)_{\Phi}=\mathbb{R} \Phi$, we have $\eta_{1}=\langle\eta, \Phi\rangle_{L^{2}} \Phi /\langle\Phi, \Phi\rangle_{L^{2}}$. Then we compute

$$
\frac{(d f \otimes d f)_{\pi(\Phi)}\left((d \pi)_{\Phi}(\eta),(d \pi)_{\Phi}(\eta)\right)}{f(\pi(\Phi))}=\frac{2\langle\eta, \Phi\rangle_{L^{2}}^{2}}{\langle\Phi, \Phi\rangle_{L^{2}}}=2\left\langle\eta, \eta_{1}\right\rangle_{L^{2}}=2\left\langle\eta_{1}, \eta_{1}\right\rangle_{L^{2}} .
$$

Then (3.8) and (5.8) imply that

$\left(\widehat{g_{I}}\right)_{\pi(\Phi)}\left((d \pi)_{\Phi}(\eta),(d \pi)_{\Phi}(\eta)\right)=2\left\langle\eta_{1}, \eta_{1}\right\rangle_{L^{2}}-\left(\left\langle\eta_{1}, \eta_{1}\right\rangle_{L^{2}}-\left\langle\eta_{35}, \eta_{35}\right\rangle_{L^{2}}\right)=\langle\eta, \eta\rangle_{L^{2}}$.

Remark 5.7. As far as the author knows, there are no known examples of an 8dimensional manifold admitting a torsion-free $\operatorname{Spin}(7)$-structure with $\operatorname{dim} \mathcal{M}_{\operatorname{Spin}(7)}=$ 2. It would be interesting to construct such examples. It is because the above pseudo-Riemannian metrics are flat by Corollary 3.17, and hence $\mathcal{M}_{\text {Spin(7) }}$ is expected to have simpler geometric structures, which might be useful to study the general cases.

The metric completion of $\mathcal{M}_{\mathrm{Spin}(7)}$ has not been studied yet. We expect that the same statements as in Remark 5.3 hold.

\subsubsection{The space of Riemannian metrics}

Let $M$ be a compact oriented $n$-dimensional manifold and let $\mathcal{M}$ be the space of all smooth Riemannian metrics on $M$. This is an open cone in the Fréchet space $\Gamma\left(S^{2} T^{*} M\right)$, the space of symmetric $(0,2)$-tensors on $M$. Thus $\mathcal{M}$ is a Fréchet manifold and its tangent space at $g \in \mathcal{M}$ is canonically identified with $\Gamma\left(S^{2} T^{*} M\right)$.

For $g \in \mathcal{M}$ and $h_{1}, h_{2} \in T_{g} \mathcal{M} \cong \Gamma\left(S^{2} T^{*} M\right)$, define a weak Riemannian metric $g_{E}$, which is called the Ebin metric, on $\mathcal{M}$ by

$$
\left(g_{E}\right)_{g}\left(h_{1}, h_{2}\right)=\int_{M} \operatorname{tr}\left(g^{-1} h_{1} g^{-1} h_{2}\right) \operatorname{vol}_{g},
$$

where $g^{-1} h_{i} \in \Gamma\left(T^{*} M \otimes T M\right)$ is the contraction of the dual Riemannian metric of $g$ and $h_{i}$, and $\operatorname{vol}_{g}$ is the volume form induced from $g$.

The local structure of $\left(\mathcal{M}, g_{E}\right)$ was first studied in [FG, GM]. The authors first proved the splitting similar to Theorem 3.3 for $\left(\mathcal{M}, g_{E}\right)$. Then they showed that the sectional curvature of $g_{E}$ is nonpositive ([FG, Corollary 1.17]) and gave the geodesics explicitly ( FG, Theorem 2.3], GM, Theorem 3.2]).

On $\mathcal{M}$, there is a canonical function $f: \mathcal{M} \rightarrow \mathbb{R}$ given by

$$
f(g)=2 \operatorname{Vol}(g)=2 \int_{M} \operatorname{vol}_{g} .
$$

Clarke showed that the pseudometric $d_{g_{E}}$ induced from $g_{E}$ is the metric in Clarke1 and determined the metric completion $\overline{\left(\mathcal{M}, d_{g_{E}}\right)}$ of $\mathcal{M}$ w.r.t. $d_{g_{E}}$ in Clarke3. 
Theorem 5.8 (Clarke3, Theorem 5.19]). Let $\mathcal{M}_{\text {finite }}$ be the set of measurable positive-semidefinite sections $g: M \rightarrow S^{2} T^{*} M$ with $f(g)<\infty$. Set $\widehat{\mathcal{M}_{\text {finite }}}=$ $\mathcal{M}_{\text {finite }} / \sim$, where $\sim$ is the equivalence relation defined by $g \sim h \Leftrightarrow g(x)=h(x)$ or $g(x) \neq h(x)$ and $\operatorname{det} g(x)=\operatorname{det} h(x)=0$ for almost everywhere $x \in M$.

Then the metric completion $\overline{\left(\mathcal{M}, d_{g_{E}}\right)}$ of $\mathcal{M}$ w.r.t. $d_{g_{E}}$ is identified with $\widehat{\mathcal{M}_{\text {finite }}}$.

For the proof, Clarke first introduced a notion of the $\omega$-convergence for Cauchy sequences in $\mathcal{M}$, which is a kind of pointwise a.e.-convergence. Then as summarized in [Clarke4, p.60], Theorem [5.8 is proved in the following steps.

(i) For any Cauchy sequence $\left\{g_{k}\right\} \subset \mathcal{M}$, there exists an $\omega$-convergent subsequence. Denote by $\left[g_{0}\right] \in \widehat{\mathcal{M}_{\text {finite }}}$ the $\omega$-limit. (ii) Two $\omega$-convergent subsequences $\left\{g_{k}^{0}\right\}$ and $\left\{g_{k}^{1}\right\}$ have the same $\omega$-limit if and only if $\left[g_{k}^{0}\right]=\left[g_{k}^{1}\right] \in$ $\overline{\left(\mathcal{M}, d_{g_{E}}\right)}$. (iii) For each element of $\mathcal{M}_{\text {finite }}$, there exists a sequence in $\mathcal{M} \omega$ converging to it.

Then a map $\overline{\left(\mathcal{M}, d_{g_{E}}\right)} \ni\left[g_{k}\right] \mapsto\left[g_{0}\right] \in \widehat{\mathcal{M}_{\text {finite }}}$ gives a bijection, where we use the notation in Definition A.2 Note that by [Clarke3, Theorem 4.21]

$$
f\left(g_{0}\right)=\lim _{k \rightarrow \infty} f\left(g_{k}\right)
$$

Using this result, Clarke and Rubinstein (CR2]) showed that $d_{g_{E} / f^{p}}$ is a metric for any $p \in \mathbb{Z}$ and determined the metric completion $\overline{\left(\mathcal{M}, d_{g_{E} / f^{p}}\right)}$ of $\mathcal{M}$ w.r.t. $d_{g_{E} / f^{p}}$.

Theorem 5.9 ([CR2, Theorem 5.3]). The metric completion $\overline{\left(\mathcal{M}, d_{g_{E} / f^{p}}\right)}$ of $\mathcal{M}$ w.r.t. $d_{g_{E} / f^{p}}$ is identified with the following.

(1) If $p=1, \widehat{\mathcal{M}_{\text {finite },+}}:=\mathcal{M}_{\text {finite },+} / \sim$, where $\mathcal{M}_{\text {finite },+}=\left\{g \in \mathcal{M}_{\text {finite }} \mid\right.$ $f(g)>0\}$.

(2) If $p<1, \widehat{\mathcal{M}_{\text {finite }} \text {. }}$

(3) If $p>1, \widehat{\mathcal{M}_{\text {finite }}+} \cup\left\{g_{\infty}\right\}$, where $g_{\infty}$ corresponds to the single equivalence class of Cauchy sequences $\left\{h_{k}\right\}$ with $\lim _{k \rightarrow \infty} f\left(h_{k}\right)=\infty$.

Now we show that we can generalize Theorem 5.9 by our method. First, we prove the following.

Proposition 5.10. The pair $\left(g_{E}, f\right)$ is a homogeneous pair of degree $n / 2$ w.r.t. the canonical $\mathbb{R}_{>0}$-action on $\mathcal{M}$.

Proof. By the definitions of $g_{E}$ and $f$, we see that (3.1) and (3.2) are satisfied for $\alpha=n / 2$. The vector field $P$ generated by the canonical $\mathbb{R}_{>0}$-action on $\mathcal{M}_{\operatorname{Spin}(7)}$ is given by $P_{g}=g$ at $g \in \mathcal{M}$. Then for any $h \in T_{g} \mathcal{M} \cong \Gamma\left(S^{2} T^{*} M\right)$ we compute

$$
(d f)_{g}(h)=\int_{M} \operatorname{tr}\left(g^{-1} h\right) \operatorname{vol}_{g}=\left(g_{E}\right)_{g}\left(P_{g}, h\right),
$$

and hence (3.3) is satisfied.

Then by Theorems $3.23,5.8$ and Proposition 3.25, we obtain the following. 
Theorem 5.11. Use the notation of Theorem [5.9, Let $v: \mathbb{R}_{>0} \rightarrow \mathbb{R}_{>0}$ be a smooth function. Let $\hat{T}_{0}$ and $\hat{T}_{\infty}$ be defined in (3.9). Then the metric completion $\overline{\left(\mathcal{M}, d_{(v \circ f) g_{E}}\right)}$ w.r.t. $(v \circ f) g_{E}$ is identified with the following.

(1) If $\hat{T}_{0}=-\infty$ and $\hat{T}_{\infty}=\infty$,

$$
\widehat{\mathcal{M}_{\text {finite },+}} \text {. }
$$

(2) If $\hat{T}_{0} \in \mathbb{R}, \hat{T}_{\infty}=\infty$ and $\lim _{r \rightarrow 0} r v(r)=0$,

$$
\widehat{\mathcal{M}_{\text {finite }}} \text {. }
$$

(3) If $\hat{T}_{0}=-\infty, \hat{T}_{\infty} \in \mathbb{R}$ and $\lim _{r \rightarrow \infty} r v(r)=0$,

$$
\widehat{\mathcal{M}_{\text {finite },}+} \cup\left\{g_{\infty}\right\} \text {. }
$$

(4) If $\hat{T}_{0} \in \mathbb{R}, \hat{T}_{\infty} \in \mathbb{R}, \lim _{r \rightarrow 0} r v(r)=0$ and $\lim _{r \rightarrow \infty} r v(r)=0$,

$$
\widehat{\mathcal{M}_{\text {finite }}} \cup\left\{g_{\infty}\right\} .
$$

Proof. By Proposition 3.25, the metric completion $\overline{\mathcal{M}_{1}}$ of $\mathcal{M}_{1}=f^{-1}(1) \subset \mathcal{M}$ w.r.t. the metric induced from the induced Riemannian metric from $g_{E}$ is homeomorphic to

$$
\left\{\left[g_{k}\right] \in \overline{\left(\mathcal{M}, d_{g_{E}}\right)} \mid \lim _{k \rightarrow \infty} f\left(g_{k}\right)=1\right\} .
$$

By the proof of Theorem 5.8 this is identified with $\widehat{\mathcal{M}_{\text {finite }, 1}}:=\mathcal{M}_{\text {finite }, 1} / \sim$, where $\mathcal{M}_{\text {finite }, 1}=\left\{g \in \mathcal{M}_{\text {finite }} \mid f(g)=1\right\}$. Then since there are canonical bijections between $\mathbb{R}_{>0} \times \overline{\mathcal{M}_{1}},\left(\{0\} \cup \mathbb{R}_{>0}\right) \times \overline{\mathcal{M}_{1}} /\left(\{0\} \times \overline{\mathcal{M}_{1}}\right),\left(\mathbb{R}_{>0} \cup\{\infty\}\right) \times$ $\overline{\mathcal{M}_{1}} /\left(\{\infty\} \times \overline{\mathcal{M}_{1}}\right),\left(\{0\} \cup \mathbb{R}_{>0} \cup\{\infty\}\right) \times \overline{\mathcal{M}_{1}} /\left(\{0, \infty\} \times \overline{\mathcal{M}_{1}}\right)$ and $\overline{\mathcal{M}_{\text {finite }}+}$, $\widehat{\mathcal{M}_{\text {finite }}}, \widehat{\mathcal{M}_{\text {finite },}} \cup\left\{g_{\infty}\right\}, \widehat{\mathcal{M}_{\text {finite }}} \cup\left\{g_{\infty}\right\}$, respectively, the proof is completed by Theorem 3.23.

Remark 5.12. This theorem generalizes Theorem 5.9. The weak Riemannian metric $(v \circ f) g_{E}$ was first considered by Bauer, Harms and Michor in [BHM]. They also considered weak Riemannian metrics weighted by scalar curvature and described the geodesic equation for these weak Riemannian metrics. Then they showed that the exponential mapping for some of them is a local diffeomorphism.

The weak Riemannian metric $g_{E} / f$ was the first example whose metric completion is strictly smaller than that of the Ebin metric $g_{E}$. We can give infinitely many such examples by Theorem 5.11 (1).

\subsubsection{The Teichmüller space}

In addition to the setting of Section 5.3.2, suppose that $M$ is a compact Riemann surface of genus $\kappa \geq 2$. Let $\mathcal{M}_{<0} \subset \mathcal{M}$ be the space of all smooth Riemannian metrics of constant negative sectional curvature on $M$.

The restrictions of $g_{E}$ and $f$ in (5.10) and (5.11) to $\mathcal{M}_{<0}$ define a Riemannianmetric and a function on $\mathcal{M}_{<0}$. These are invariant under the action of $\operatorname{Diff}_{0}(M)$, 
the identity component of the diffeomorphism group. Thus they induce a Riemannian metric and a function on $\mathcal{T}_{<0}:=\mathcal{M}_{<0} / \operatorname{Diff}_{0}(M)$. By an abuse of notation, we denote these by $g_{E}$ and $f$. Proposition 5.10 implies that $\left(g_{E}, f\right)$ is a homogeneous pair of degree 1 w.r.t. the canonical $\mathbb{R}_{>0}$-action on $\mathcal{M}_{<0}$.

By the Gauss-Bonnet formula, we have

$$
K_{g} \cdot f(g)=2 \int_{M} K_{g} \operatorname{vol}_{g}=4 \pi(2-2 \kappa) .
$$

for $g \in \mathcal{M}_{<0}$. Thus setting $l=8 \pi(\kappa-1)$, we have

$$
\mathcal{T}_{l}:=\left\{[g] \in \mathcal{T}_{<0} \mid f([g])=l\right\}=\left\{[g] \in \mathcal{T}_{<0} \mid K_{g}=-1\right\},
$$

which is called the Teichmüller space of $M$. The induced Riemannian metric on $\mathcal{T}_{l}$ from $g_{E}$ is called the Weil-Petersson metric.

This space is well understood. The space $\mathcal{T}_{l}$ is known to be a $(6 \kappa-6)$ dimensional manifold homeomorphic to $\mathbb{R}^{6 \kappa-6}$. Since $g_{E}$ and $f$ are invariant under the action of $\operatorname{Diff}_{+}(M)$, where $\operatorname{Diff}_{+}(M)$ is the group of orientation preserving diffeomorphisms of $M$, they induce a Riemannian metric and a function on the orbifold $\mathcal{M}_{<0} / \operatorname{Diff}_{+}(M)=\mathcal{T}_{<0} / \operatorname{MCG}(M)$, where $\operatorname{MCG}(M)=$ $\operatorname{Diff}_{+}(M) / \operatorname{Diff}_{0}(M)$ is the mapping class group. By an abuse of notation, we denote these by $g_{E}$ and $f$. Then the metric completion of $\mathcal{T}_{l} / \operatorname{MCG}(M)$ w.r.t. the metric induced from $g_{E}$ is homeomorphic to the Deligne-Mumford compactification of the moduli space of Riemann surfaces of genus $\kappa$, which is a projective algebraic variety.

The statements in this paper would be true for orbifolds. On the orbifold $\mathcal{M}_{<0} / \operatorname{Diff}_{+}(M)=\mathcal{T}_{<0} / \operatorname{MCG}(M),\left(g_{E}, f\right)$ is a homogeneous pair of degree 1 w.r.t. the canonical $\mathbb{R}_{>0}$-action on $\mathcal{M}_{<0} / \operatorname{Diff}_{+}(M)$ by Proposition 5.10, Then we have the metric completion as in Theorem 3.23 In particular, for a function $v: \mathbb{R}_{>0} \rightarrow \mathbb{R}_{>0}$ corresponding to the case (4) in Theorem 3.23 the metric completion of $\mathcal{M}_{<0} / \operatorname{Diff}_{+}(M)$ w.r.t. the metric induced from $(v \circ f) g_{E}$ will be compact by Remark 3.24. It will be interesting if we can know that the metric completion of $\mathcal{M}_{<0} / \operatorname{Diff}_{+}(M)$ is also a projective algebraic variety for some $v$.

\section{A Appendix}

We summarize the notations and basic definitions used in this paper.

Definition A.1. Let $(M, g)$ be a pseudo-Riemannian manifold. We call a pseudo-Riemannian metric definite if it is positive or negative definite.

Definition A.2. Let $(Z, d)$ be a metric space. The metric completion $\bar{Z}$ w.r.t. the metric $d$ is defined by $\bar{Z}=Z_{C} / \sim$, where $Z_{C}$ is the space of Cauchy sequences in $Z$ and $\sim$ is the equivalence relation defined by $\left\{z_{k}\right\} \sim\left\{z_{k}^{\prime}\right\} \Leftrightarrow$ $\lim _{k \rightarrow \infty} d\left(z_{k}, z_{k}^{\prime}\right)=0$. Denote by $\left[z_{k}\right]$ the equivalence class of $\left\{z_{k}\right\}$. Then $\bar{Z}$ is a metric space with the metric $d\left(\left[z_{k}\right],\left[z_{k}^{\prime}\right]\right)=\lim _{k \rightarrow \infty} d\left(z_{k}, z_{k}^{\prime}\right)$, where we also use $d$ to describe the metric on $\bar{Z}$ by an abuse of notation.

We summarize the notations used in this paper. In the following table, $(M, g)$ is a pseudo-Riemannian manifold. 


\begin{tabular}{|c|c|}
\hline Notation & Meaning \\
\hline$\overline{\mathbb{R}_{>0}}$ & $\overline{\mathbb{R}_{>0}=\{x \in \mathbb{R} \mid x>0\}}$ \\
\hline$i(\cdot)$ & The interior product \\
\hline$|v|_{g}$ & $|v|_{g}=\sqrt{g(v, v)}$ for $v \in T M$ when $g$ is positive-definite \\
\hline$d_{g}$ & The induced (pseudo)metric from $g$ when $g$ is positive-definite \\
\hline $\bar{M}$ & The metric completion of $M$ w.r.t. $d_{g}$ when $g$ is positive-definite \\
\hline$\left[x_{k}\right]$ & The equivalence class in $\bar{M}$ of a Cauchy sequence $\left\{x_{k}\right\} \subset M$ \\
\hline $\begin{array}{l}\operatorname{grad}^{g} f \\
v^{b} \in T^{*} M\end{array}$ & $\begin{array}{l}\text { The gradient vector field of a function } f \text { defined by } g\left(\operatorname{grad}^{g} f, \cdot\right)=d f \\
v^{b}=g(v . \cdot) \text { for } v \in T M\end{array}$ \\
\hline$\alpha^{\sharp} \in T M$ & $\alpha=g\left(\alpha^{\sharp}, \cdot\right)$ for $\alpha \in T^{*} M$ \\
\hline$\nabla^{g}$ & The Levi-Civita connection of $g$ \\
\hline$R^{g}$ & The curvature tensor: $R^{g}(A, B)=\left[\nabla_{A}^{g}, \nabla_{B}^{g}\right]-\nabla_{[A, B]}^{g}$ for $A, B \in T M$ \\
\hline$K^{g}$ & The sectional curvature of $g$ \\
\hline $\mathfrak{X}(M)$ & The space of smooth vector fields on $M$ \\
\hline$\rho^{\prime}, \rho^{\prime \prime}, \cdots$ & For a function of one variable $\rho=\rho(r), \rho^{\prime}=d \rho / d r, \rho^{\prime \prime}=d^{2} \rho / d r^{2}, \cdots$ \\
\hline $\begin{array}{l}\dot{r}, \ddot{r}, \cdots \\
\partial_{r}\end{array}$ & $\begin{array}{l}\text { For a function of one variable } r=r(t), \dot{r}=d r / d t, \ddot{r}=d^{2} r / d t^{2}, \cdots \\
\partial_{r}=\partial / \partial r\end{array}$ \\
\hline$K^{g}\left(\partial_{r}\right)$ & $K^{g}\left(\partial_{r}\right)=\frac{-2 \rho^{\prime \prime} \xi+\rho^{\prime} \xi^{\prime}}{2 \rho \xi^{2}}$ defined in (2.8) \\
\hline$E_{1}$ & $E_{1}=g_{Y}\left(\dot{y}_{0}, \dot{y}_{0}\right)\left(\rho\left(r_{0}\right)\right)^{4}$ defined in Proposition 2.4 \\
\hline$E_{2}$ & $E_{2}=\xi\left(r_{0}\right)\left(\dot{r}_{0}\right)^{2}+g_{Y}\left(\dot{y}_{0}, \dot{y}_{0}\right)\left(\rho\left(r_{0}\right)\right)^{2}$ defined in Lemma 2.11 \\
\hline$E_{3}$ & $E_{3}=\rho\left(r_{0}\right)^{2}$ defined in Proposition 2.4 \\
\hline$F$ & $F=\frac{1}{4}\left(\left(\frac{\dot{r}_{0}}{r_{0}}\right)^{2}+\frac{g_{Y}\left(\dot{y}_{0}, \dot{y}_{0}\right)}{k}\right)$ defined in Proposition 2.18 \\
\hline$g(w)$ & $g(w)=\frac{k w(r)}{r^{2}} d r^{2}+w(r) g_{Y}$ given in (2.15) \\
\hline
\end{tabular}

\section{References}

[AN] S. Amari and H. Nagaoka, Methods of information geometry, Translations of Mathematical Monographs 191, AMS, Oxford, Univ. Press.

[AJLS] N. Ay, J. Jost, H.V. Lê and L. Schwachhöfer, Information geometry, Springer, Cham, 2017.

[BHM] M. Bauer, P. Harms, P.W. Michor, Sobolev metrics on the Riemannian manifold of all Riemannian metrics, J. Differential Geom. 94 (2013), $187-208$.

[Clarke1] B. Clarke, The metric geometry of the manifold of Riemannian metrics over a closed manifold, Calc. Var. Partial Differential Equations 39 (2010), 533-545.

[Clarke2] B. Clarke, The Riemannian $L^{2}$ topology on the manifold of Riemannian metrics, Ann. Glob. Anal. Geom. 39 (2011), 131-163.

[Clarke3] B. Clarke, The completion of the manifold of Riemannian metrics, J. Differential Geom. 93 (2013), 203-268.

[Clarke4] B. Clarke, Geodesics, distance, and the CAT(0) property for the manifold of Riemannian metrics, Math. Z. 273 (2013), 55-93. 
[CR1] B. Clarke an Y. A. Rubinstein, Ricci flow and the metric completion of the space of Kähler metrics, Amer. J. Math. 135 (2013), 1477-1505.

[CR2] B. Clarke an Y. A. Rubinstein, Conformal deformations of the Ebin metric and a generalized Calabi metric on the space of Riemannian metrics, Ann. Inst. H. Poincaré Anal. NonLinéaire 30 (2013), 251-274.

[Dubrovin] B. Dubrovin, On Almost Duality for Frobenius Manifolds, Amer. Math. Soc. Transl. 212 (2004), 75-132.

[FG] D.S. Freed, D. Groisser, The basic geometry of the manifold of Riemannian metrics and of its quotient by the diffeomorphism group, Mich. Math. J. 36 (1989), 323-344.

[GM] O. Gil-Medrano, P.W. Michor, The Riemannian manifold of all Riemannian metrics, Q. J. Math. 42 (1991), 183-202.

[GY] S. Grigorian and S.-T. Yau, Local geometry of the $G_{2}$ moduli space, Commun. Math. Phys. 287 (2009), 459-488.

[Grigorian] S. Grigorian, Moduli spaces of $G_{2}$ manifolds, Rev. Math. Phys. 22 (2010), 1061-1097.

[Hitchin1] N. Hitchin, The moduli space of special Lagrangian submanifolds, Ann. Scuola Norm. Sup. Pisa Cl. Sci. 25 (1997), 503-515.

[Hitchin2] N. Hitchin, The geometry of three-forms in six and seven dimensions, J. Differential Geom. 55 (2000), 547-576.

[Huybrechts] D. Huybrechts, Products of harmonic forms and rational curves, Doc. Math. 6 (2001), 227-239.

[Joyce1] D. D. Joyce, Compact 8-manifolds with holonomy Spin(7), Invent. Math. 123 (1996), 507-552.

[Joyce2] D. D. Joyce, Compact Riemannian 7-manifolds with holonomy $G_{2}$. I, J. Differential Geom. 43 (1996), 291-328.

[Joyce3] D. D. Joyce, Compact manifolds with special holonomy, Oxford Mathematical Monographs, Oxford University Press, Oxford, 2000.

[Karigiannis] S. Karigiannis, Deformations of $\mathrm{G}_{2}$ and $\operatorname{Spin}(7)$-structures on Manifolds, Canad. J. Math. 57 (2005), 1012-1055.

[KL] S. Karigiannis and N. C. Leung, Hodge Theory for $G_{2}$-manifolds: Intermediate Jacobians and Abel-Jacobi Maps, Proc. Lond. Math. Soc. (3) 99 (2009), 297-325.

[KLL] S. Karigiannis, C. Lin, and J. Loftin, Octonionic-algebraic structure and curvature of the Teichmüller space of $G_{2}$ manifolds, in preparation.

[KN] S. Kobayashi and K. Nomizu, Foundations of Differential Geometry, Vol I, Wiley-Interscience, New York, 1963.

[KM] A. Kriegl and P. W. Michor, The Convenient Setting of Global Analysis, Math. Surveys Monogr. 53, AMS, Providence, RI, 1997. 
[Lang] S. Lang, Introduction to differentiable manifolds Second edition, Universitext. Springer-Verlag, New York, 2002.

[Loftin] J. Loftin, Affine spheres and Kähler-Einstein metrics, Math. Res. Lett. 9 (2002), 425-432.

[Magnússon] G. Magnússon, The geometry of Kähler cones, arXiv:1211.6934

[MM] P. W. Michor and D. Mumford, Vanishing geodesic distance on spaces of submanifolds and diffeomorphisms, Doc. Math. 10 (2005), 217-245.

[O'Neill] B. O'Neill, Semi-Riemannian Geometry with Applications to Relativity, Academic Press, New York, 1983.

[Shima] H. Shima, The Geometry of Hessian Structures, World Scientific, Singapore, 2007.

[Totaro] B. Totaro, The curvature of a Hessian metric, Internat. J. Math. 15 (2004), 369-391.

[TW] T. Trenner and P. M. H. Wilson, Asymptotic curvature of moduli spaces for Calabi-Yau threefolds, J. Geom. Anal. 21 (2011), 409-428.

[Wilson] P. M. H. Wilson, Sectional curvatures of Kähler moduli, Math. Ann. 330 (2004), 631-664.

Gakushuin University, 1-5-1, Mejiro, Toshima,Tokyo, 171-8588, Japan

E-mail address: kkawai@math.gakushuin.ac.jp 